

\section{ASIM 2022}

www.asim-gi.org/asim2022

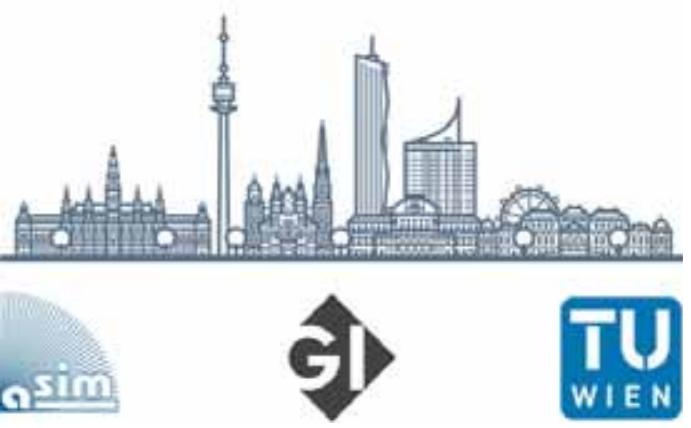

ASIM , die Arbeitsgemeinschaft Simulation, lädt zu einer Tagung ein, wie es früher einmal war:

ASIM 2022 - 26. Symposium Simulationstechnik 14.-16. Februar 2022, TU Wien

Das Symposium ASIM 2022 wird organisiert durch die TU Wien, in Kooperation mit der DLR und der Hochschule Wismar, sowie mit allen Fachgruppen der ASIM. Diese Tagung, nach langer Zeit wieder mit persönlicher Teilnahme, ist neben der internationalen Konferenz MATHMOD Vienna 2022 das Hauptereignis der ASIM im Jahr 2022 und inkludiert auch das alljährliche Workshop der ASIM Fachgruppen GM MS (Grundlagen und M ethoden) und STS (Technische Systeme).

ASIM 2022 - von 14.2. mittags bis 16.2. mittags - findet unmittelbar vor der M ATHMOD Vienna 2022 (10th Vienna International Conference on Mathematical Modelling, 16.-18. Februar 2022, www.mathmod.at) statt, wodurch sich viele Synergien ergeben.

Das Themenspektrum reicht von den Grundlagen, Methoden und Werkzeugen der Modellbildung und Simulation über alle Anwendungsbereiche von den Natur- und Ingenieurwissenschaften über Informatik, Produktion und Logistik, Bio-, Umwelt- und Geowissenschaften, Klima- und Ökosystemmodellen bis zur Ausbildung in Modellierung und Simulation und deren Nutzung in der Ausbildung. Parallele, spezialisierte Sessions ermöglichen einen intensiven, spezifischen und gebietsübergreifenden Erfahrungsaustausch.

Wir laden herzlich zur Einreichung von Beiträgen ein, die - wie auch die Tagungspräsentationen - in Deutsch oder Englisch verfasst werden können. ASIM 2022 bietet zwei Arten von Beiträgen, die einem Peer Review Prozess unterliegen:

- Langbeiträge: 6 - 8 Seiten, deutsch oder englisch, elektronische Publikation mit individueller DOI im Tagungsband Langbeiträge ASIM 2022, ARGESIM Verlag, ARGESIM Report 20, ASIM M itteilung 180, ISBN ebook 978-3-901608-97-1; Präsentation: 20 M inuten Ausgewählte Beiträge werden zur Veröffentlichung im Fachjournal Simulation Notes Europe (SNE) vorgeschlagen.

- Kurzbeiträge: 2 - 4 Seiten, deutsch oder englisch, elektronische Publikation im Tagungsband Kurzbeiträge ASIM 2022, ARGESIM Verlag, ARGESIM Report 19, ASIM M itteilung 179; ISBN ebook 978-3-901608-96-4; Präsentation: 20 M inuten

\section{Information / Kontakt}

www.asim-gi.org/asim2022

E-Mail: asim2022@asim-gi.org 


\section{Editorial}

Dear Readers, This issue SNE 31(2) mainly publishes post-conference contributions from ASIM conferences and workshopsshowing the broad variety of classical simulation areas and looking into new areas.

The first contribution by D. Lückerath et al. introduces the ARCH project, with its goal of saving cultural heritage from climaterelated and other hazards by means of data simulation - providing also the title graphic for this SNE issue.

The following two contributions are more of technical nature: F. Klabunde et al. make use of simulation for all-electric machines and renewable electric power systems in agricultural operations, and R. Courant and J. Mass present an orthotropic non-hysteretic permeability model as a lookup-table for FEA. The next two contributions deal with simulation software: P. Junglas combines mealy behaviour and causality in NSA-DEVS, and P. Abele and M. Schäfer present SyNumSeS, a Python package for numerical simulation of semiconductor devices. F. S. Pappert's and O. Rose's contribution addresses big data and simulation, by reducing response time with data farming and machine learning. And the last Technical Note by L.V. Hanisch and M. Henke deals with the unjustifiable poor cousin of simulation, with design of experiments - the authors investigate lifetime modelling of electrical machines using the methodology of design of experiments

The last contribution is a Benchmark Note. In 2019, SNE has introduced extended possibilities for submitting a benchmark contribution: i) a Benchmark Solution, ii) or a Benchmark Report, iii) or a Benchmark Study, differing in details and approach, and in publication length. Additionally, content may concentrate on educational aspects (Educational Benchmark Note 'BNE'), and student authors may submit a benchmark contribution from their views (Student Benchmark Note - BNS). And so, a group of master students has realised with 'A Tutorial-oriented Approach to ARGESIM Benchmark C11 'SCARA Robot' in MATLAB, Simulink and Stateflow' an Educational Student Benchmark Note, from students for students, or as handout for exercise work.

I would like to thank all authors for their contribution, and thanks to the editorial board members for review and support, and to the organizers of the EUROSIM conferences for co-operation in post-conference contributions. And many thanks to the SNE Editorial Office for layout, typesetting, preparations for printing, electronic publishing, and much more.

And last but not least we are glad, that after the series of virtual conferences we can announce in this issue two EUROSIM conferences as they used to be, MATHMOD 2022 and ASIM 2022 - Face-to-Face conferences in February 2022.

Felix Breitenecker, SNE Editor-in-Chief,eic@sne-journal.org; felix.breitenecker@tuwien.ac.at

\section{Contents SNE 31(2)}

Online SNE 31(2), DOI 10.11128/sne.31.2.1056

ARGESIM Publisher, Vienna, www.argesim.org

\section{Print SNE 31(2) ISBN 978-3-903311-16-9}

TU Verlag Vienna, Print-on-Demand, www.tuverlag.at

The ARCH Project: Investigating the Impact of Climate Change on Historic Areas. D. Lückerath, K. Milde, O. Ullrich $\quad \ldots \quad 53$

Modelling and Simulation of All-Electric Machines and Renewable Electric Power Systems in Agricultural Operations. F. Klabunde, C. Reinhold, B. Engel

Orthotropic Non-hysteretic Permeability Model as a Lookup-Table for FEA. R. Courant, J. Maas

NSA-DEVS: Combining Mealy Behaviour and Causality. P. Junglas

SyNumSeS: A Python Package for Numerical Simulation of Semiconductor Devices. P. Abele, M. Schäfer

Reducing Response Time with Data Farming and Machine Learning. F. S. Pappert, O. Rose

Lifetime Modelling of Electrical Machines using the Methodology of Design of Experiments. L. V. Hanisch, M. Henke

A Tutorial-oriented Approach to ARGESIM Benchmark C11 'SCARA Robot' in MATLAB, Simulink and Stateflow. .J. Leindecker, M. Zechmeister-Machhart, F. Gauss, P.Wiegard Back Cover

\section{SNE Contact \& Info}

\section{SNE Online ISSN 2306-0271, SNE Print ISSN 2305-9974}

$\rightarrow$ www.sne-journal.org

㪯= office@sne-journal.org, eic@sne-journal.org

$\square$ SNE Editorial Office

Johannes Tanzler (Layout, Organisation),

Irmgard Husinsky (Web, Electronic Publishing),

Felix Breitenecker (Organisation, Author M entoring) ARGESIM / M ath. M odelling \& Simulation Group, Inst. of Analysis and Scientific Computing, TU Wien Wiedner Hauptstrasse 8-10, 1040 Vienna, Austria

\section{SNE SiMULATION NOTES EUROPE}

WEB: $\rightarrow$ www.sne-journal.org, DOI prefix 10.11128/sne Scope: Developments and trends in modelling and simulation in various areas and in application and theory; comparative studies and benchmarks (documentation of ARGESIM Benchmarks on modelling approaches and simulation implementations); modelling and simulation in and for education, simulation-based e-learning; society information and membership information for EUROSIM members (Federation of European Simulation Societies and Groups).

Editor-in-Chief: Felix Breitenecker, TU Wien, M ath. M odelling Group 奉=-Felix.Breitenecker@tuwien.ac.at, 跮= eic@sne-journal.org Print SNE and Print-on-Demand: TU-Verlag, Wiedner Hauptstrasse 8-10, 1040, Vienna, Austria - www.tuverlag.at Publisher: ARGESIM ARBEITSGEM EINSCHAFT SIMULATION NEWS c/o Math. Modelling and Simulation Group, TU Wien / 101, Wiedner Hauptstrasse 8-10, 1040 Vienna, Austria; www.argesim.org, $=$ = info@argesim.org on behalf of ASIM www.asim-gi.org and EUROSIM $\rightarrow$ www.eurosim.info (c) ARGESIM / EUROSIM / ASIM 2021 


\section{SNE - Aims and Scope}

Simulation Notes Europe (SNE) provides an international, high-quality forum for presentation of new ideas and approaches in simulation - from modelling to experiment analysis, from implementation to verification, from validation to identification, from numerics to visualisation - in context of the simulation process.

SNE seeks to serve scientists, researchers, developers and users of the simulation process across a variety of theoretical and applied fields in pursuit of novel ideas in simulation and to enable the exchange of experience and knowledge through descriptions of specific applications. SNE follows the recent developments and trends of modelling and simulation in new and/or joining application areas, as complex systems and big data. SNE puts special emphasis on the overall view in simulation, and on comparative investigations, as benchmarks and comparisons in methodology and application. For this purpose, SNE documents the ARGESIM Benchmarks on Modelling Approaches and Simulation Implementations with publication of definitions, solutions and discussions. SNE welcomes also contributions in education in/for/with simulation.

A News Section in SNE provides information for EUROSIM Simulation Societies and Simulation Groups.

SNE, primarily an electronic journal, follows an open access strategy, with free download in basic layout. SNE is the official membership journal of EUROSIM, the Federation of European Simulation Societies and Simulation Groups - www.eurosim.info. Members of EUROSIM societies are entitled to download SNE in an elaborate and extended layout, and to access additional sources of benchmark publications, model sources, etc. Print SNE is available for specific groups of EUROSIM societies, and starting with Volume 27 (2017) as printon-demand from TU Verlag, TU Wien. SNE is DOI indexed by CrossRef, identified by DOI prefix 10.11128, assigned to the SNE publisher ARGESIM (www.argesim.org).

Author's Info. Individual submissions of scientific papers are welcome, as well as post-conference publications of contributions from conferences of EUROSIM societies. SNE welcomes special issues, either dedicated to special areas and/or new developments, or on occasion of events as conferences and workshops with special emphasis.

Authors are invited to submit contributions which have not been published and have not being considered for publication elsewhere to the SNE Editorial Office.

SNE distinguishes different types of contributions (Notes), i.e.

- TN Technical Note, 6 - 10 p. $\quad$ EN Education Note -6 - 8 p.

- PN Project Note 6-8 p. - SN Short Note, max. 6 p.

- SW Software Note, 4-6p. - BN Benchmark Note, 2-8p.

- ON Overview Note - only - BNE Edu B.Note, $210 p$ upon invitation, up to $14 \mathrm{p}$. $\quad$ STN Student Note, 4-8 p, inv.

Further info and templates (doc, tex) at SNE's website. www.sne-journal.org

\section{SNE Editorial Board}

SNE - Simulation Notes Europe is advised and supervised by an international scientific editorial board. This (increasing) board is taking care on peer reviewing of submission to SNE:

Felix Breitenecker, Felix.Breitenecker@tuwien.ac.at TU Wien, Math. Modelling, Austria, Editor-in-chief David Al-Dabass, david.al-dabass@ntu.ac.uk, Nottingham Trent University, UK

Maja Atanasijevic-Kunc,maja.atanasijevic@fe.uni-lj.si Univ. of Ljubljana, Lab. Modelling \& Control, Slovenia

Aleš Belič, ales.belic@sandoz.com Sandoz / National Inst. f. Chemistry, Slovenia

Peter Breedveld, P.C.Breedveld@el.utwente.nl University of Twente, Netherlands

Agostino Bruzzone,agostino@itim.unige.it Universita degli Studi di Genova, Italy

Francois Cellier, fcellier@inf.ethz.ch, ETH Zurich, Switzerland

Vlatko Čerić,vceric@efzg.hr, Univ. Zagreb, Croatia

Russell Cheng,rchc@maths.soton.ac.uk University of Southampton, UK

Roberto Cianci, cianci@dime.unige.it, Math. Eng. and Simulation, Univ. Genova, Italy

Eric Dahlquist, erik.dahlquist@mdh.se, Mälardalen Univ., Sweden Umut Durak, umut.durak@dlr.de German Aerospace Center (DLR)Braunschweig, Germany

Horst Ecker,Horst.Ecker@tuwien.ac.at TU Wien, Inst. f. Mechanics, Austria

Vadim Engelson,vadime@mathcore.com MathCore Engineering, Linköping, Sweden

Peter Groumpos,groumpos@ece.upatras.gr Univ. of Patras, Greece

Edmond Hajrizi, ehajrizi@ubt-uni.net University for Business and Technology, Pristina, Kosovo

Glenn Jenkins, GLJenkins@cardiffmet.ac.uk Cardiff Metropolitan Univ., UK

Emilio Jiménez, emilio.jimenez@unirioja.es University of La Rioja, Spain

Esko Juuso,esko.juuso@oulu.fi Univ. Oulu, Dept. Process/Environmental Eng., Finland

Kaj Juslin, kaj.juslin@enbuscon.com, Enbuscon Ltd, Finland Andreas Körner, andreas.koerner@tuwien.ac.at TU Wien, Math. E-Learning Dept., Vienna, Austria

Francesco Longo,f.longo@unical.it Univ. of Calabria, Mechanical Department, Italy Yuri Merkuryev,merkur@itl.rtu.lv, Riga Technical Univ.

David Murray-Smith,d.murray-smith@elec.gla.ac.uk University of Glasgow, Fac. Electrical Engineering, UK

Gasper Music,gasper.music@fe.uni-lj.si Univ. of Ljubljana, Fac. Electrical Engineering, Slovenia

Thorsten Pawletta, thorsten.pawletta@hs-wismar.de Univ. Wismar, Dept. Comp. Engineering, Wismar, Germany Niki Popper, niki.popper@dwh.at, dwh Simulation Services, Austria

Kozeta Sevrani, kozeta.sevrani@unitir.edu.al Univ. Tirana, Inst.f. Statistics, Albania

Thomas Schriber, schriber@umich.edu University of Michigan, Business School, USA

Yuri Senichenkov, sneyb@dcn.infos.ru St. Petersburg Technical University, Russia

Michal Štepanovský, stepami9@fit.cvut.cz Technical Univ. Prague, Czech Republic

Oliver Ullrich, oliver.ullrich@iais.fraunhofer.de Fraunhofer IAIS, Germany

Siegfried Wassertheurer, Siegfried.Wassertheurer@ait.ac.at AIT Austrian Inst. of Technology, Vienna, Austria

Sigrid Wenzel, S.Wenzel@uni-kassel.de Univ. Kassel, Inst. f. Production Technique, Germany Grégory Zacharewicz, gregory.zacharewicz@mines-ales.fr IMT École des Mines d'Alès, France 


\title{
The ARCH Project: Investigating the Impact of Climate Change on Historic Areas
}

\author{
Daniel Lückerath*, Katharina Milde, Oliver Ullrich
}

Fraunhofer Institute for Intelligent Analysis and Information Systems IAIS, Schloss Birlinghoven, 53757 Sankt Augustin, Germany; *daniel.lueckerath@iais.fraunhofer.de

SNE 31(2), 2021, 53 - 56, DOI: 10.11128/sne.31.sn.10561

Received: May 16, 2021; Revised May 26, 2021;

Accepted: June 3, 2021

SNE - Simulation Notes Europe, ARGESIM Publisher Vienna,

ISSN Print 2305-9974, Online 2306-0271, www.sne-journal.org

Abstract. This short contribution introduces the EU Horizon 2020 project $A R C H$ "Advancing resilience of historic areas against climate-related and other hazards" [1]. It summarizes ARCH's objectives, briefly introduces the piloting city cases, describes the work undertaken in the first 18 months of the project, and briefly touches on how these results go beyond the state-of-the-art.

\section{Context and Objectives}

Historic towns, old urban quarters, villages, and hamlets, as well as historic landscapes make up a significant part of Europe's identity: Natural heritage sites cover roughly $18 \%$ of the European land territory [2], and on average $22 \%$ of the European housing stock was constructed before 1946 [3]. These historic areas are deeply embedded in larger urban and rural environments, serving a role in preserving local identity and personality as well as local knowledge. Although climate change has become one of the most significant and fastest growing threats to people and their cultural heritage [4] the impacts of climate-related and other natural hazards on historic areas have not been studied extensively [5], and disaster risk reduction seldom registers as a priority area for management of World Heritage property [6].

Therefore, there is a need for specific methods and tools that provide better information and decision-support for climate change adaptation and disaster risk reduction for heritage managers, urban planners, policymakers, and the general public. These methods and tools need to take into account the unique physical, environmental, economic, social, cultural, and political aspects of historic areas, as well as the enabling conditions these areas provide for taking action.

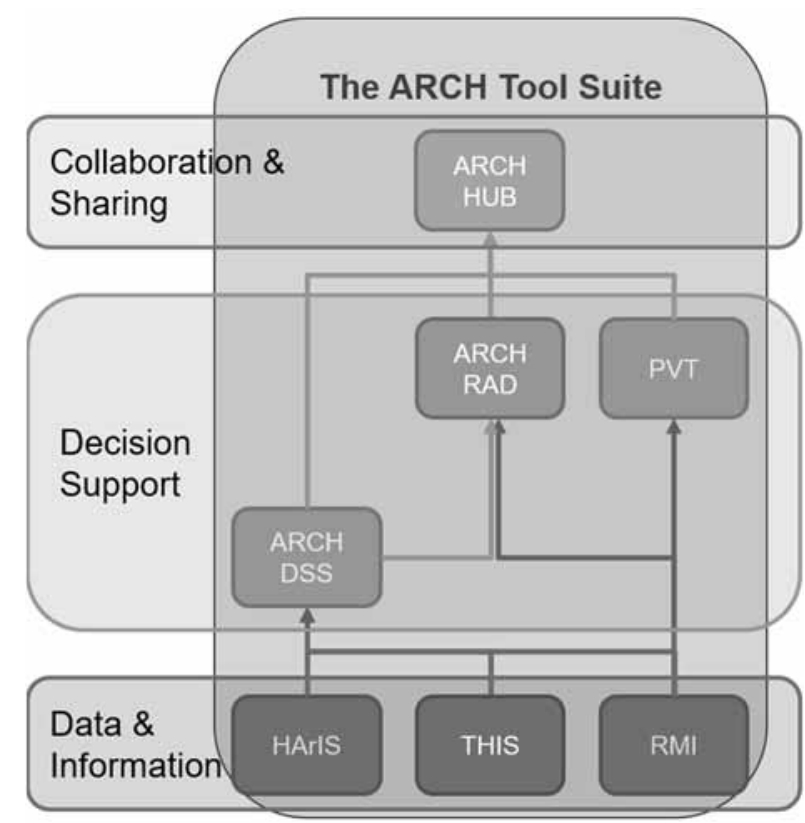

Figure 1: ARCH develops a collaborative, web-based disaster risk management plaform for urban heritage districts.

However, tools and methods alone are not enough. They need to be supported by a stronger promotion of relevant public policies and participatory governance processes that facilitate the active participation of residents from local communities and the general public. The awareness of climate change impacts on historic areas needs to be increased, resilience building strategies need to be included in heritage management policies and practices, while at the same time the role of heritage in climate change adaptation and disaster risk reduction within the wider urban context has to be emphasized.

The EU Horizon 2020 research project ARCH aims to take a step in this direction. ARCH will provide a suite of software tools (see Figure 1) for assessing and improving the resilience of historic areas, conceptualized as part of three layers: The Data and Information Layer includes information on cultural heritage as part of the Historic 
Area Information System (HArIS), on specific challenges resulting from climate change as part of the Threats and Hazards Information System (THIS), and of suitable resilience enhancing measures as part of the the Resilience Measures Inventory (RMI).

These information repositories will be made available to practitioners through web tools on the Decision Support Layer. That layer includes the GIS-based ARCH Decision Support System (ARCH DSS) used to monitor natural phenomena and events in real time, to predict damage scenarios and assess the risks of corresponding impacts and consequences, the Resilience Assessment Dashboard (RAD) enabling local stakeholders to perform a structured assessment of their municipality's resilience against the impact of climate change, and the Pathway Visualization Tool (ARCH PVT) allowing the visual design of implementation pathways for resilienc measures. All of these are concentrated and provided by one single collaboration-oriented, user-friendly web site, the ARCH HUB [7].

While providing local practitioners with these tools, at the same time ARCH will advocate new policies as well as necessary changes to existing regulations for heritage management, climate change adaptation, and disaster risk reduction of historic areas.

The ARCH solutions will target heritage managers, urban planners, and other professionals from the fields of climate change adaptation and disaster risk reduction as well as politicians and the general public, in order to improve the cooperation and communication between these stakeholders and to enhance the definition and implementation of sustainable resilience building strategies.

\section{City Cases}

To assure the best possible understanding of the specific impact of climate change on local communities, and at the same time ensuring maximum applicability of the developed methods and tools, all ARCH research and development is conducted in co-creation with four municipal partners [8], Bratislava (Slovakia), Camerino (Italy), Hamburg (Germany), and Valencia (Spain). These four pilot cities, located all over Europe and facing related, but different specific challenges, work in concert with researchers and SMEs to find solutions focused on the protection of historic areas from the impact of climate change as well as of other natural hazards.
Bratislava. The historical center of Bratislava, the capital city of Slovakia, has become a tourist magnet in recent years. However, with its high housing density, including numerous in-situ preserved Celtic industrial monuments located underground, relatively few urban green areas, and its location along the foothills of the Small Carpathians, pluvial flooding resulting from extreme precipitation events has become a major problem. In addition to examining the extent of and potential solutions to that hazard, the ARCH project also examines the local Devin Castle located on a steep cliff on the Danube river (see Figure 2). Climate change induced changes to freeze and thaw cycles as well as increases in humidity cause erosion effects to the cliff that threaten the rock face to collapse into the river, taking the castle with it.
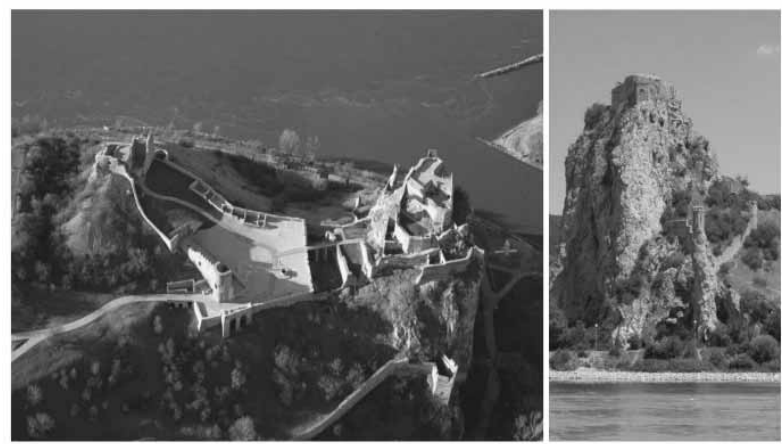

Figure 2: Bratislava's Devin Castle perched on an eroding cliff on the Danube river (Source: [10])

Camerino. Camerino in the Marche region of central Italy is a historic town that was hit badly by a series of major earthquakes in 2016. Its main historic area, located on a hill top and including the cathedral, the Palazzo Ducale and a - up until then - mostly historically intact walled town center are still recovering from that event and are for the most part uninhabitable. With the Camerino city case the ARCH project demonstrates that its methods and tools, mainly designed and developed to mitigate the impact of climate change, can also be applied to help in the aftermath of other natural disasters.

Hamburg. With the interplay of the German Bight's tides and the river Elbe changing slowly as a result of climate change and the increased demands of servicing bigger ships in the harbour, Hamburg and its Speicherstadt World Heritage Site face new challenges: Rows and rows of warehouses were built from 1883 to 1927 on pine pile foundations. 
Resulting from prolonged intervals during which the pole heads are outside of the tidal-range, the load bearing properties of these pine poles might slowly but surely decrease, causing the heritage site to subside. As part of the $\mathrm{ARCH}$ project risks and vulnerabilities due to these effects are assessed on neighborhood as well as single building level. In addition, the monitoring and management of the historic warehouses is supported via advanced $3 \mathrm{D}$ models.

Valencia. Located at the Mediterraean coast, the city of Valencia is surrounded on three sides by the Huerta de Valencia, a peri-urban farmland recognized as Globally Important Agricultural Heritage System by the Food and Agriculture Organization of the United Nations (FAO). L'Horta, as well as Albufera national park, the second examination site for ARCH in Valencia, provides important recreational and economic value to the city in addition to being a huge asset to mitigate the effects of climate change. However, with increases in temperature and drought as well as rising sea levels both cultural landscapes are at serious risk. ARCH provides specialized risk climate services and risk analysis models focusing on agro-climatic changes to L'Horta and waterquality changes to the Albufera Lagoon.

\section{Results Achieved so far}

ARCH started off with the production of a series of stateof-the-art reports of concepts, approaches, standards, and technologies on topics crucial for the project [9]. These reports dealt with, amongst other things, the current state of conservation practices, the concepts of disaster risk management and building back better, gender mainstreaming, decision-support frameworks, as well as existing standards related to ARCH's topics. Some of the key findings from these reports include:

- Cultural heritage management and disaster risk management remain poorly integrated.

- An indicator-based approach to risk assessments seems most appropriate for historic areas.

- Disaster risk management, for historic areas hinges critically on the inclusion of local communities, local practices, and traditional knowledge.

- Users need to be able to understand the decision process, limitations, and associated uncertainties of a Decision Support System (DSS).
In parallel to the state-of-the-art reports, four City Baseline Reports were compiled that establish the baseline for the municipalities of Bratislava, Camerino, Hamburg, and Valencia, with respect to their selected historic areas [10]. As ARCH has a multi-dimensional thematic focus on heritage management, disaster risk reduction and adaptation to climate change, all three dimensions were addressed in each city's baseline review.

Based on these reports, project partners conducted match-making meetings with each city to match local problems with specific solutions provided by ARCH. These meetings, as well as all co-creation activities of the project, are governed by an ARCH co-creation guideline document that presents a harmonised methodological approach and framework for the cooperation between scientific partners and a city.

In addition to the match-making meetings, each ARCH pilot city identified 'local partnerships' and developed a work plan for local activities, aligned with the research work of the project. [11]

ARCH also looked beyond its pilot cities and compiled a report that maps and characterises European initiatives and case studies on advancing the resilience of cultural heritage to natural and human-induced disasters as well as to the long-term consequences of climate change.

Of the 40 initiatives featured in this report, 32 were described as snapshots and eight as more detailed case studies. [12]

In order to ensure that the solutions developed by $\mathrm{ARCH}$ are in line with the needs of local end-users, as expressed in the match-making sessions, and with current practices, a requirements analysis was conducted and system specifications were drawn up. ARCH partners identified 134 requirements across the four pilot cities, each mapped to one or more of the solutions that will be developed by the ARCH project partners. [13]

Based on the exploratory work, ARCH has developed a combined disaster risk management (DRM) and climate change adaptation cycle, the ARCH Disaster Risk Management Framework.

The ARCH DRM Framework is specifically aimed at historic areas and helps actors in the fields of heritage management, disaster risk management, and climate change adaptation to understand which steps are necessary to develop a combined disaster risk management and climate change adaptation plan with specific focus on historic areas. [14] 


\subsection{Progress beyond the state-of-the-art}

Although only halfway through the lifetime of the project, with some work packages still in the early stages of work, there is already significant impact and progress beyond the state of the art:

- The ARCH DRM Framework successfully combines the Disaster Risk Management Cycle with the Climate Change Adaptation planning cycle, also including issues of heritage management and social justice.

- The replicability framework developed for the mapping and characterisation of European initiatives and case studies provides an easily usable blueprint for identification of initiatives that might be suited to be transferred to other contexts.

- The ARCH co-creation guideline provides a practical handbook on how to conduct successful co-creation in many types of applied research projects. [15]

- The establishment of local partnerships in each pilot city and the ongoing co-creation process has already resulted in higher visibility of the issues addressed by ARCH within the city administrations and larger stakeholder networks, e.g. by establishing committed local cross-departmental partnerships that will potentially live beyond the lifetime of the project.

- The involvement of ARCH partners in formal standardisation processes on national and international level brings the project very close to influencing the state-of-the-art in standardisation fields related to the project topics.

\section{Acknowledgements}

This paper is an updated and extended version of the conference short paper "ARCH - Saving Cultural Heritage from Climate-related and Other Hazards" by Daniel Lückerath, Katharina Milde, and Oliver Ullrich that was published as part of the proceedings of ASIM-Workshop STS/GMMS 2021. It has been prepared in the framework of the European project ARCH - Advancing Resilience of historic areas against Climate-related and other Hazards. This project has received funding from the European Union's Horizon 2020 research and innovation programme under grant agreement no. 820999.

The sole responsibility for the content of this publication lies with the authors. It does not necessarily represent the opinion of the European Union. Neither the EASME nor the European Commission are responsible for any use that may be made of the information contained therein.

\section{References}

[1] ARCH Advancing Resilience of Historic Areas Against Climate-related and Other Hazards, https://savingculturalheritage.eu/, accessed on Jan. 26, 2021.

[2] European Commission. Europe's Cultural and natural Heritage in Natura 2000. Publications Office of the European Union. Luxembourg; 2018.

[3] Nicol S, Roys M, Ormandy D., Ezratty V. The cost of poor housing in the European Union. University of Warwick; 2016.

[4] ICOMOS, 19GA 2017/30, Resolutions of the $19^{\text {th }}$ General Assembly, Dec 2017; New Delhi, India.

[5] Bigio A, Ochoa M C, Amirtahmasebi R. Climate-resilient, Climatefriendly World Heritage Cities. Urban Development Series Knowledge Papers, Bd. 19, World Bank, Washington, DC, 2014.

[6] Global Platform for Disaster Risk Reduction, Heritage and Resilience. Issues and Opportunities for reducing disaster risks, Global Platform for Disaster Risk Reduction. Geneva, Switzerland; 2013.

[7] ARCH HUB. EU H2020 ARCH (GA No. 820,999), https://hub.savingculturalheritage.eu, accessed on May 25, 2021.

[8] Ullrich O, Bogen M, Lückerath D, Rome E. Co-operating with Municipal Partners on Indicator Identification and Data Acquisition. Simulation Notes Europe (SNE), Volume 29, Number 4, pp. 159-168, 2019.

[9] Lückerath D, et al. State-of-the-art reports of concepts, approaches, standards and technologies. EU H2020 ARCH (GA No. 820,999), Deliverable D7.1, 2019.

[10] Streberova E, Mastrocola B, Mense U, Garcia L, et al. City baseline report. EU H2020 ARCH (GA No. 820,999), Deliverable D3.3, 2020.

[11] Chapman E, et al. Local partnership and work plan. EU H2020 ARCH (GA No. 820,999), Deliverable D3.2, 2020.

[12] Rebollo V, Latinos V.Good practices in building cultural heritage resilience. EU H2020 ARCH (GA No. 820,999), Deliverable D7.2, 2020.

[13] Nickel K, Milde K, Lückerath D, Rome E. End-user requirements to ARCH tools and methologies. EU H2020 ARCH (GA No. 820,999), Deliverable D7.4, 2020.

[14] Milde K, Lückerath D, Ullrich O. ARCH Disaster Risk Management Framework. EU H2020 ARCH (GA No. 820,999), Deliverable D7.3, 2020.

[15] Latinos V, Chapman E. Guideline on ARCH co-creation approach. EU H2020 ARCH (GA No. 820,999), Deliverable D3.1, 2020. 


\title{
Modelling and Simulation of All-Electric Machines and Renewable Electric Power Systems in Agricultural Operations
}

\author{
Felix Klabunde*, Christian Reinhold, Bernd Engel \\ elenia Institute for High Voltage Technology and Power Systems, Technische Universität Braunschweig, \\ Schleinitzstrasse 23, 38106 Braunschweig, Germany; ${ }^{*}$.klabunde@tu-braunschweig.de
}

SNE 31(2), 2021, 57-64, DOI: 10.11128/sne.31.tn.10562 Received: May 23, 2021 (Selected ASIM SST 2020 Postconf. Publ.), Accepted: May 31, 2021

SNE - Simulation Notes Europe, ARGESIM Publisher Vienna, ISSN Print 2305-9974, Online 2306-0271, www.sne-journal.org

Abstract. Today's operation of agricultural machines is still characterised by the use of fossil fuels. In the future, all-electric agricultural machines can be used, whose energy requirements must be covered by local renewable energies or the power grid. The occurrence of limit value violations in the power grid can slow down and hinder this change in agricultural operations. For this reason, this paper describes the modelling and simulation of allelectric agricultural machines, renewable energy systems and rural power grids and the evaluation of suitable energy supply strategies for the machines and needed investments in the power grid.

\section{Introduction}

The restructuring of the German energy system in the context of the Energiewende requires the interaction and commitment of private and commercial stakeholders. As one of the main players in decentralized energy production, today's agriculture already has a special role in the Energiewende. In addition to the widespread photovoltaic plants, agriculturally operated biogas plants can be used to provide weatherindependent electrical and thermal power. The combination of different generation and storage systems leads to a variety of energy structures with different technical and economic potential. In combination with new electricity applications in agriculture, such as all-electric agricultural machines, these energy structures have the potential to make agriculture sustainable in the long term. To determine the technical-economic potential and to derive suitable recommendations for action for the involved stakeholders, it is necessary to carry out holistic modelling and simulation of the involved technologies. Therefore, this paper describes the modelling and simulation of agricultural influenced energy systems and power grids with a focus on all-electric agricultural machines. In a simulation of different scenarios, suitable energy supply strategies are derived.

\section{All-electric Agricultural Machines}

More and more manufacturers of agricultural machinery have turned their attention to all-electric agricultural machines in recent years. Given the further development of power electronics and electric motors, as well as new requirements in efficiency and environmental protection, a future use of all-electric agricultural machines is realistic and comprehensible. For many processes in field cultivation, there are currently only prototypes and concepts and no marketready products, which is why the modelling approach of all-electric agricultural machines, as shown in Figure 1 , is based on empirical values and consumption profiles of diesel-powered agricultural machines. The

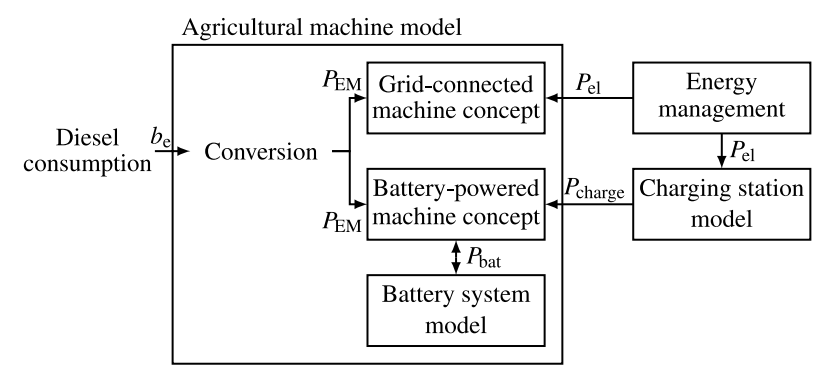

Figure 1: Modelling approach for the all-electric agricultural machines. 
diesel consumption profiles $b_{e}$ are converted to electrical power values $P_{E M}$, which represent the power requirement of the electric drive train. These electrical consumption profiles serve as input data for the two modelled machine concepts: the battery-powered agricultural machine and the grid-connected agricultural machine. The battery-powered agricultural machine is recharged by the charging station with the charging power $P_{\text {charge }}$. The charging station as well as the gridconnected agricultural machine are connected to the energy management system via their electrical power consumption $P_{\mathrm{el}}$. The conversion process as well as both machine concepts are explained in more detail below.

\subsection{Conversion of the diesel consumption profiles}

The diesel consumption profiles used in this paper were created in the EkoTech research project [1] and represent a typical agricultural process chain in field cultivation. The process chain includes the agricultural processes soil tillage, sowing, plant protection, fertilisation and harvesting. While three agricultural machines are used simultaneously for harvesting (harvester and two tractors for transporting the harvest), only one agricultural machine is used at a time for the other processes. The use of diesel consumption profiles offers the advantage of using both simulated consumption profiles as well as measured consumption profiles. By using time series instead of static data, the varying power requirements of the individual processes can be realistically represented and process-dependent statements can be made regarding the machine concept to be used and the necessary parameterisation of the agricultural machine. The conversion to an electric power profile is done by using the calorific value of diesel $H_{i}$ and overall efficiency of the diesel-powered drive train $\eta_{\mathrm{CM}}$ with $P_{E M}$ representing the power consumption of the electric drive train and $b_{e}$ representing the diesel consumption rate:

$$
P_{E M}=b_{e} \cdot H_{i} \cdot \eta_{\mathrm{CM}}
$$

With the calorific value of diesel of $9.86 \mathrm{kWh} / \mathrm{l}$ and an assumed static efficiency of the diesel-powered drive train of $30 \%$, the electric motor output, shown in Figure 2 for the process soil tillage, is obtained. Depending on the agricultural process, the energy and power requirements of the agricultural machines vary greatly. Particularly in field cultivation, many processes are associated with a high energy input. Machines used for soil tillage, sowing and as harvester in harvesting have

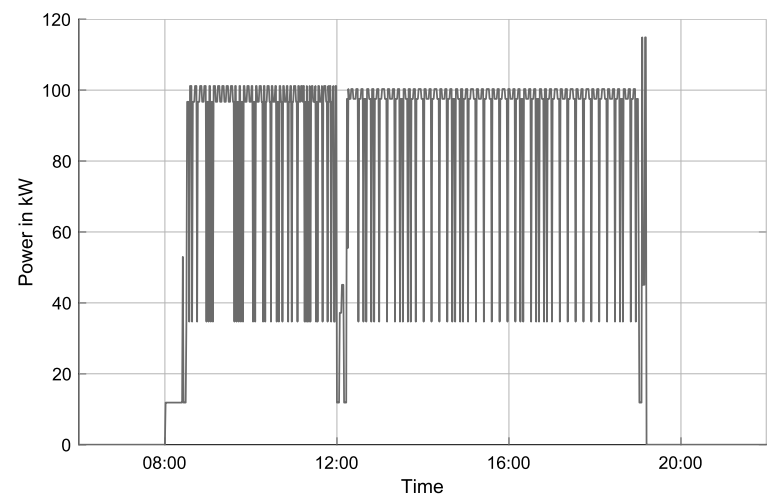

Figure 2: Resulting power consumption $P_{\mathrm{EM}}$ of the electric drive train during soil tillage.

a high energy consumption of up to 1 MWh per day, while other processes have a comparatively low energy consumption (e.g. the resulting electrical energy consumption for one tractor for transporting the harvest is $115 \mathrm{kWh}$ per day). Due to the different requirements it is essential to consider different machine concepts with different parameterization for all-electric agricultural machines.

\subsection{Battery-powered agricultural machine}

Battery-powered agricultural machines draw their energy from a battery system, which can be installed in the machine or as exchangeable front/back weight. The advantage of the battery-powered concept lies in its unlimited mobility and the possibility of bidirectional charging.

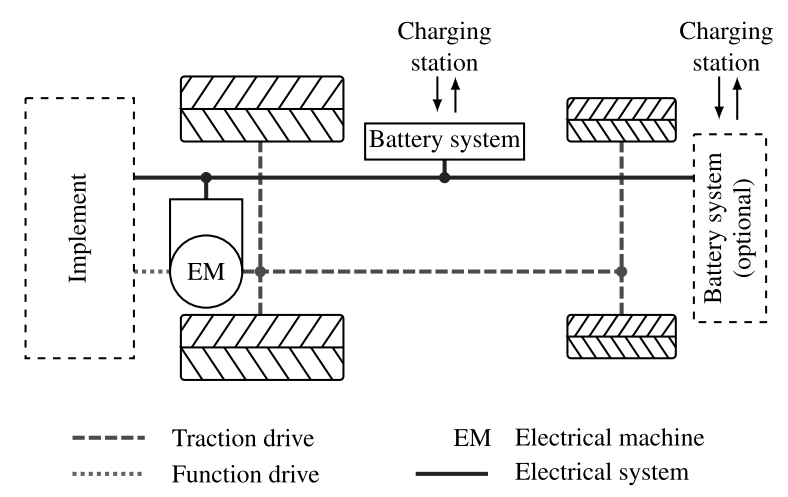

Figure 3: Possible machine concept of a battery-powered agricultural machine.

The battery-powered agricultural machine is modelled by a battery system which is discharged by the specified electrical consumption profile and recharged by a model of a charging station. The presence of the agri- 
cultural machine and the maximum possible recharging time at the charging station is determined by the power consumption of the electric drive train $P_{\mathrm{EM}}$. If no power is needed $\left(P_{\mathrm{EM}}=0\right)$ it is assumed for simplification that the agricultural machine is at the charging station and has the possibility of recharging at the charging station's full nominal power. The presence of the agricultural machine at the charging station is described by the parameter appearance:

$$
P_{\mathrm{EM}}\left\{\begin{array}{l}
>0 \rightarrow \text { appearance }=0 \\
=0 \rightarrow \text { appearance }=1
\end{array}\right.
$$

The charging power at the charging station $P_{\text {charge }}$ is calculated depending on the chosen charging strategy (Charging with maximum power, Charging with minimum power) and the maximum charging power of the battery system:

$$
P_{\text {charge }}=\min \left\{\begin{array}{l}
P_{\text {bat, }, \text { charge, } m a x} \\
P_{\text {charge,strategy }}
\end{array}\right.
$$

$P_{\text {bat,charge,max }}$ is the maximum charging power of the battery system and $P_{\text {charge,strategy }}$ is the charging power set by the charging strategy. $P_{\text {bat,charge,max }}$ is calculated depending on the state-of-charge of the battery and is based on typical battery charging profiles. In the event that the actual state-of-charge is smaller than the stateof-charge at cut-off voltage, the charging power is calculated using the following equation:

$$
P_{\text {bat,charge,max }}=\frac{C \cdot E_{\text {bat,nom }}}{\eta_{\text {bat,charge }}}
$$

$C$ is the charging/discharging rate, $E_{\text {bat,nom }}$ is the nominal battery capacity, $E_{\text {bat }}$ is the available battery capacity and $\eta_{\text {bat,charge }}$ is the charging efficiency of the battery system. If the battery voltage reaches the cut-off voltage, the charging current is reduced at constant battery voltage, resulting in a reduced charging power. This effect is implemented by adjusting equation 4 :

$$
P_{\text {bat,charge,max }}=\frac{C \cdot E_{\text {bat,nom }}}{\eta_{\text {bat,charge }}} \cdot\left(\frac{\mathrm{SOC}_{\text {max }}-\mathrm{SOC}}{\mathrm{SOC}_{\max }-\mathrm{SOC}_{\mathrm{cov}}}\right)
$$

SOC is the available state-of-charge, $\mathrm{SOC}_{\max }$ is the maximum state-of-charge and $\mathrm{SOC}_{\mathrm{cov}}$ is the state-ofcharge at cut-off voltage.

In the case of the loading strategy Charging with maximum power, the agricultural machine is reloaded with the nomimal charging power of the charging station $P_{\text {charge,nom }}$ after completion of the field cultivation:

$$
P_{\text {charge,strategy }}=P_{\text {charge, } \max }=P_{\text {charge,nom }}
$$

This charging strategy enables the agricultural machine to be recharged quickly, reducing the required idle time of the agricultural machine and increasing the potential operating time. The second charging strategy, Charging with minimum power, calculates the minimum charging power as a function of the total idle time $T_{\text {appearance }}$ as shown in the following equations and Figure 4.

$$
\begin{aligned}
E_{\mathrm{bat}, \text { dest }} & =E_{\mathrm{bat}, \text { nom }}-E_{\mathrm{bat}} \\
P_{\text {charge,strategy }} & =P_{\text {charge,min }}=\frac{E_{\mathrm{bat}, \text { dest }}}{T_{\text {appearance }} \cdot \eta_{\text {charge }}}
\end{aligned}
$$

$E_{\text {bat,dest }}$ is the required battery capacity to be recharged, $E_{\text {bat }}$ is the available battery capacity and $P_{\text {charge,min }}$ is the minimum charging power. This charging strategy is dependent on the next operating time of the agricultural machine and potentially reduces load peaks by recharging evenly over the entire idle time of the agricultural machine.

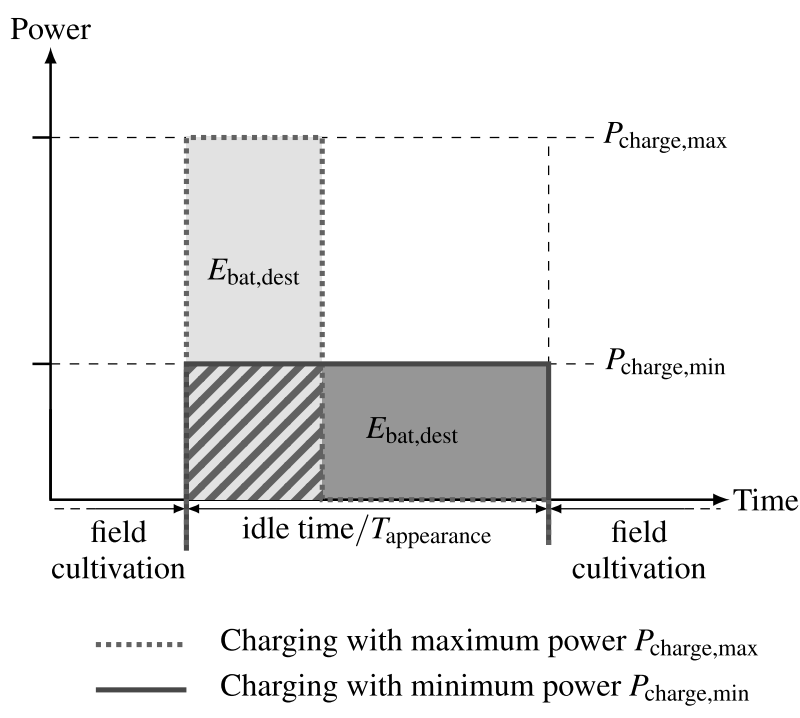

Figure 4: Influence of the charging strategies on charging power and time.

For the discharge power of the battery system, it is assumed for simplification that the power consumption $P_{\mathrm{EM}}$ can be covered at any time. This assumption is sufficient, as it can be assumed that only agricultural machines that can provide the required electrical power are used. 


\subsection{Grid-connected agricultural machine}

Grid-connected agricultural machines are continuously supplied with energy via a power cable and do not have their own energy storage. Higher theoretical engine power is advantageous than with battery-powered agricultural machines, but mobility is restricted due to the cable, which is why this concept is not suitable for every agricultural process. The concept is particularly suitable for the power-intensive soil tillage and for the harvester in harvesting. In contrast to the battery-powered agricultural machine, the engine power $P_{\mathrm{EM}}$ is directly transmitted as output power $P_{\mathrm{el}}$ to the energy management.

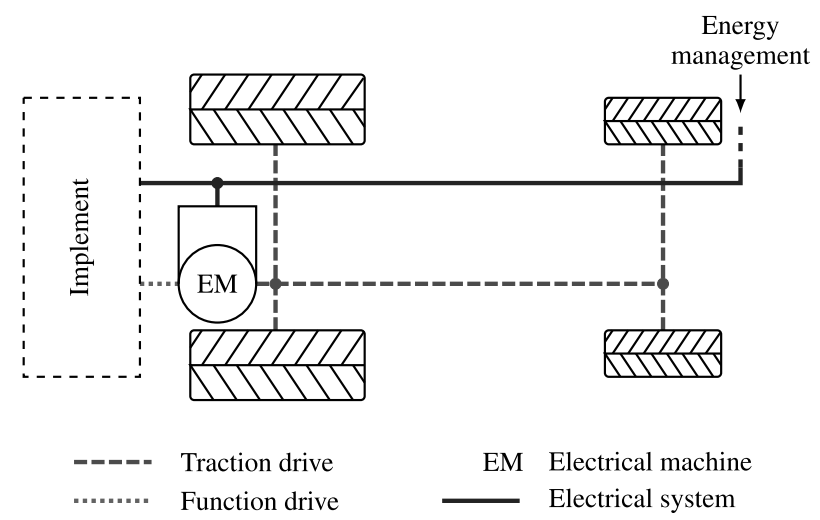

Figure 5: Machine concept of a grid-connected agricultural machine.

\section{Modelling of the Renewable Electric Power System}

All-electric agricultural machines can be supplied with energy from renewable energy plants operated by agricultural operations or from the power grid. To assess the effects of the integration of these machines and to derive suitable energy supply concepts, the modelling of the plants under consideration is necessary. In the following, the modelling of the photovoltaic plant, wind power plant and biogas plant as considered renewable energies, as well as the energy management and power grid, is briefly explained.

\subsection{Renewable Energies}

The photovoltaic model builds on the work already done in [2] and is based on a physics-based modelling approach. Weather data (solar radiation, solar azimuth, solar altitude, outdoor temperature and cloud cover) from the test reference year of the german weather service [3] and serve as input data.

A data-based modelling approach as shown in Figure 6 , is chosen for the wind turbine model, in which the generation capacity is calculated based on weather data from [4] and wind power curves from product data sheets of wind turbine manufacturers (e.g. [5])

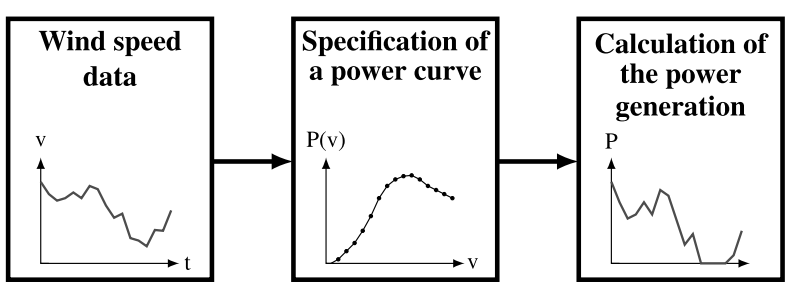

Figure 6: Data-driven modelling approach of the wind power plant.

The biogas plant is modelled in a simplified way using the load profile E0 of E-Control (austrian government regulator for electricity and gas) [6]. This simplification is possible if biogas plants are operated at constant generation capacity without a flexilibilty option.

\subsection{Energy management and power grid}

The energy management serves as a superordinate control system of the power flows between the individual models on the farm and provides the interface to the power grid. The control process aims to maximise the internal consumption of the energy generated by the renewable energies and thus reduce the amount of energy supplied by the grid.

The grid calculation is carried out with the help of MATPOWER [7]. MATPOWER calculates the power flow with the grid structure and generator and consumption power as input data. The grid results can then be analysed and visualised in MATLAB and required and suitable grid optimisation and reinforcement measures can be determined.

\section{Simulation Environment}

The modelling of the technical components (Chapter 2) and the simulation studies (Chapter 4) are carried out in the institute's simulation environment eSE (elenia Simulation Environment) [8]. eSE is a MATLAB-based simulation environment for the scientific investigation of electrical and thermal systems and their behavior. In 
addition, a flexible signal coupling of individual devices enables the investigation of connected systems and the testing of control concepts.

The simulation environment is divided into different modules, which can be used independently or in combination. The connection between all modules is the Simulator, which is represented by a single MATLAB class. It controls the information flow between the modules and takes over the central data management. The result of a simulation is a collection of time series of individual information flows and physical parameters of the devices, which can then be analyzed and evaluated. Figure 7 shows the three pathes of the simulation process in eSE.

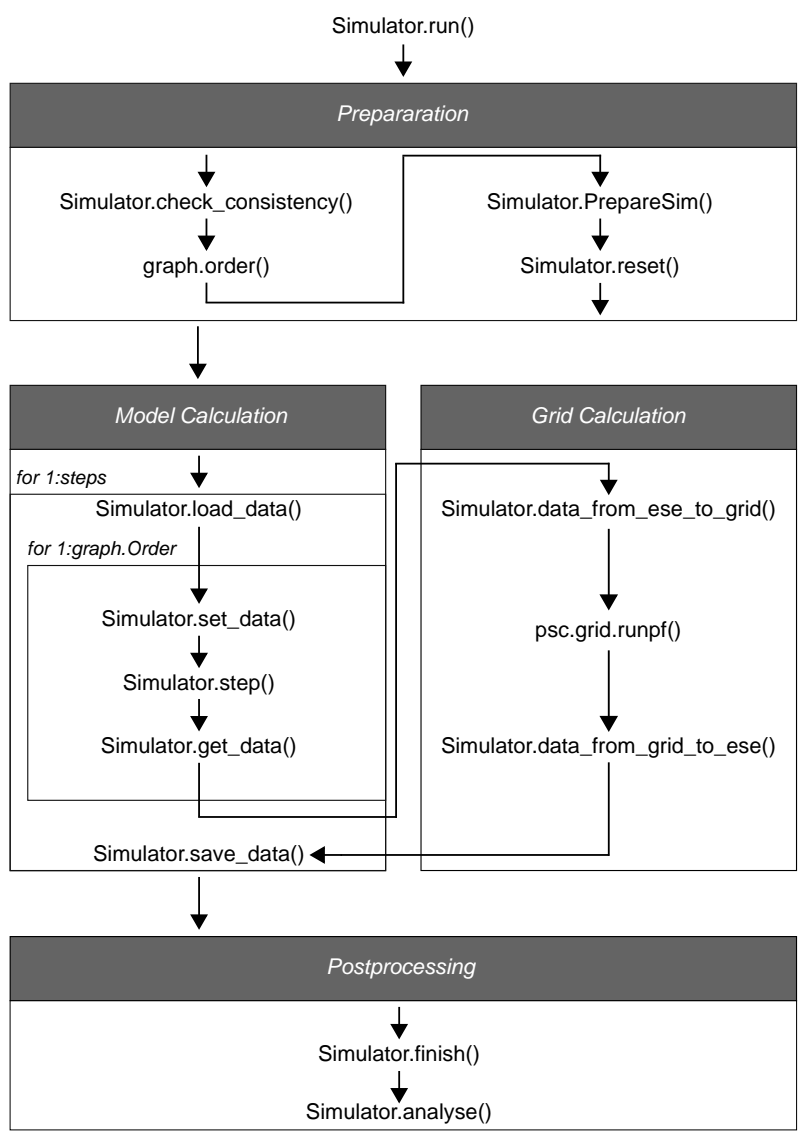

Figure 7: Simulation process in eSE.

In the preparation step, the simulation is checked for consistency and possible errors and automatically repaired if necessary. Afterwards a directed graph is created, which indicates the simulation order of the models by means of a topological search algorithm. Before all models are reset to a valid initial state, the simulation is prepared. During the preparation, model relationships are stored temporarily in a local data structure to avoid database queries during the simulation.

At the beginning of each simulation step, stored external data sets are reloaded from an HDF5 file. Subsequently, three methods are executed for each model step. Before the model calculation, data from other models or external data sets are assigned to the model. Then the model with the functional relationships is calculated and result values are queried afterwards. The result values for active and reactive power are used as input values for the grid calculation to perform the load flow calculation with MATPOWER. The resulting values of the grid resources are finally queried and stored together with the model results in the HDF5 file for time series.

In the final phase, defined key indicators of time series and model properties are calculated and stored in an SQLite database. In addition, economic calculations of stakeholders and models are executed.

\section{Simulation Scenarios and Results}

To assess the power grid integration for all-electric agricultural machines, several simulation scenarios are considered and compared with each other. The simulation scenarios differ according to the power grid connection level of the agricultural enterprise (low voltage or medium voltage) and the selected charging strategy (Charging with minimum power, Charging with maximum power), resulting in four simulation scenarios. Due to the differentiation at the power grid connection level, different compositions of renewable energies are considered for the scenarios. The selected parameterization is shown in Table 1.

\begin{tabular}{c|c|c}
\hline Model & $\begin{array}{c}\text { Low voltage } \\
\text { scenarios }\end{array}$ & $\begin{array}{c}\text { Medium voltage } \\
\text { scenarios }\end{array}$ \\
\hline Photovoltaic plant & $30 \mathrm{~kW}$ & $100 \mathrm{~kW}$ \\
\hline Wind power plant & - & $100 \mathrm{~kW}$ \\
\hline Biogas plant & - & $75 \mathrm{~kW}$ \\
\hline Charging Station & $50 \mathrm{~kW}$ & $300 \mathrm{~kW}$ \\
\hline $\begin{array}{c}\text { Battery-powered } \\
\text { machines }\end{array}$ & $\begin{array}{c}1 \times 260 \mathrm{kWh} \\
1 \times 600 \mathrm{kWh}\end{array}$ & $1 \times 260 \mathrm{kWh}$ \\
\hline $\begin{array}{c}\text { Grid-connected } \\
\text { machines }\end{array}$ & 2 & $\mathrm{kWh}$ \\
\hline
\end{tabular}

Table 1: Parameterization of the simulation scenarios. 
For all scenarios, four all-electric agricultural machines are considered, whereby two are battery-powered with built-in batteries (used in fertilization, sowing and harvest) and two are grid-connected (used in soil tillage and harvest). The inclusion of four agricultural machines in the scenario enables a realistic representation of agricultural machines needed for a typical process chain in field cultivation. The battery-powered agricultural machines can be recharged after completion of the field cultivation at one charging station. A realistic annual load profile from a measurement campaign on a dairy farm is used as base load to model the remaining electricity consumption of the agricultural operation [9].

The low voltage distribution grid is represented by Kerber's Landnetz Kabel 1 benchmark power grid and supplies five agricultural operations and three households [10]. The medium voltage distribution grid used is the CIGRE benchmark grid, where at load nodes 8 and 11 an agricultural operations is considered [11]. The remaining load nodes are mapped with standard load profiles for households and businesses [12].

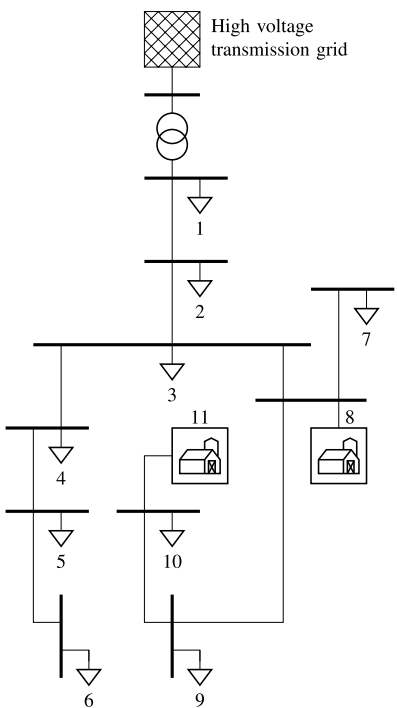

Medium voltage distribution grid

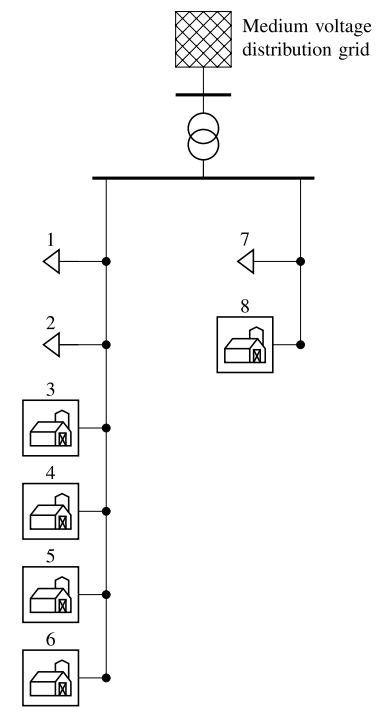

Low voltage distribution grid
Figure 8: Structure of the modelled distribution grids.

The simulation is carried out using nine randomly selected type days distributed over an entire year, which allows seasonally dependent fluctuations in generation and consumption to be depicted and meaningful results to be obtained for the entire year. The type days cover arbitrarily selected agricultural processes with each agricultural machine being used at least once. The

\begin{tabular}{c|c|c|c}
\hline Season & Day & $\begin{array}{c}\text { Frequency } \\
\text { per year }\end{array}$ & $\begin{array}{c}\text { Agricultural } \\
\text { process }\end{array}$ \\
\hline \multirow{3}{*}{ Winter } & 13.03. & 100 & Fertilization \\
& 23.11. & 20 & - \\
& 03.02 & 20 & Plant protection \\
\hline \multirow{5}{*}{ Summer } & 14.08. & 88 & Harvest \\
& 03.08. & 18 & Soil tillage \\
& 26.05. & 17 & - \\
\hline \multirow{3}{*}{ Transition } & 09.04. & 73 & - \\
& 20.04. & 14 & Fertilization \\
& 15.09. & 15 & Sowing \\
\hline
\end{tabular}

Table 2: Chosen type days for the simulation.

frequency of the type days does not necessarily correspond to the whole duration of the agricultural processes, but since the agricultural machines are mostly used for further agricultural work, the selection of processes and allocation to the type days is assumed to be sufficiently accurate.

\subsection{Technical results}

The simulated agricultural operation with low voltage power grid connection shows an average own consumption of $39 \%$ and an average self-sufficiency of $17 \%$ when using the charging strategy Charging with maximum power. On days without the use of all-electric

\begin{tabular}{l|l|l}
\hline & $\begin{array}{l}\text { Charging } \\
\text { max. Power }\end{array}$ & $\begin{array}{l}\text { Charging } \\
\text { min. Power }\end{array}$ \\
\hline Own consumption & $39 \%$ & $39 \% \Rightarrow$ \\
\hline Self-sufficiency & $17 \%$ & $18 \%$ \\
\hline max. power grid supply & $212 \mathrm{~kW}$ & $188 \mathrm{~kW}$ \\
\hline max. power grid feed-in & $22 \mathrm{~kW}$ & $22 \mathrm{~kW} \Rightarrow$ \\
\hline
\end{tabular}

Table 3: Technical results for the agricultural operations with low voltage power grid connection.

agricultural machines, but with a high level of renewable energy production, there are feed-in capacities of up to a maximum of $22 \mathrm{~kW}$. On days with usage of all-electric agricultural machines and low regenerative energy production, a grid supply of up to $212 \mathrm{~kW}$ is required. By using the charging strategy Charging with minimum power, the own consumption and selfsufficiency do not increase significantly, as the charging 
times of the battery-powered agricultural machines are outside the generation times of the photovoltaic plant for both charging strategies.

\begin{tabular}{l|l|l}
\hline & $\begin{array}{l}\text { Charging } \\
\text { max. Power }\end{array}$ & $\begin{array}{l}\text { Charging } \\
\text { min. Power }\end{array}$ \\
\hline Own consumption & $17 \%$ & $18 \%$ \\
\hline Self-sufficiency & $70 \%$ & $98 \%$ \\
\hline max. power grid supply & $233 \mathrm{~kW}$ & $123 \mathrm{~kW}$ \\
\hline max. power grid feed-in & $178 \mathrm{~kW}$ & $178 \mathrm{~kW}$ \\
\hline
\end{tabular}

Table 4: Technical results for the agricultural operations with medium voltage power grid connection.

In the two scenarios with grid connection to the medium voltage distribution grid, the degree of self-sufficiency can be significantly increased, which can be attributed to the additional consideration of a biogas plant and wind power plant and the increased nominal capacity of the photovoltaic plant. Using the charging strategy Charging with minimum power instead of Charging with maximum power, the degree of self-sufficiency can be increased to nearly $100 \%$, since the generation capacity of the biogas plant and wind power plant can cover the entire consumption capacity more frequently (cf. Figure 9).

For the scenarios considered, possible voltage band violations and thermal overloads in the power grid are examined. The voltage applied to the network nodes in the power grid changes depending on the connected loads, generation plants and the location in the power grid. The permitted deviations from the nominal voltage are defined in DIN EN 50160 [13].

The thermal load capacity of the power grid elements used is regarded as a further key figure for determining the condition of the power grid. The nominal load capacity of the equipment is often specified in the associated data sheets and is assumed to be the limit value in the following.

\begin{tabular}{l|l|l}
\hline & $\begin{array}{l}\text { Charging } \\
\text { max. Power }\end{array}$ & $\begin{array}{l}\text { Charging } \\
\text { min. Power }\end{array}$ \\
\hline Voltage range deviations & $18 \%$ & $18 \% \Rightarrow$ \\
\hline Thermal overload & $21 \%$ & $18 \%$ \\
\hline
\end{tabular}

Table 5: Frequency of limit violations in the low voltage power grid per year.

Table 5 shows the results for the low voltage distribution
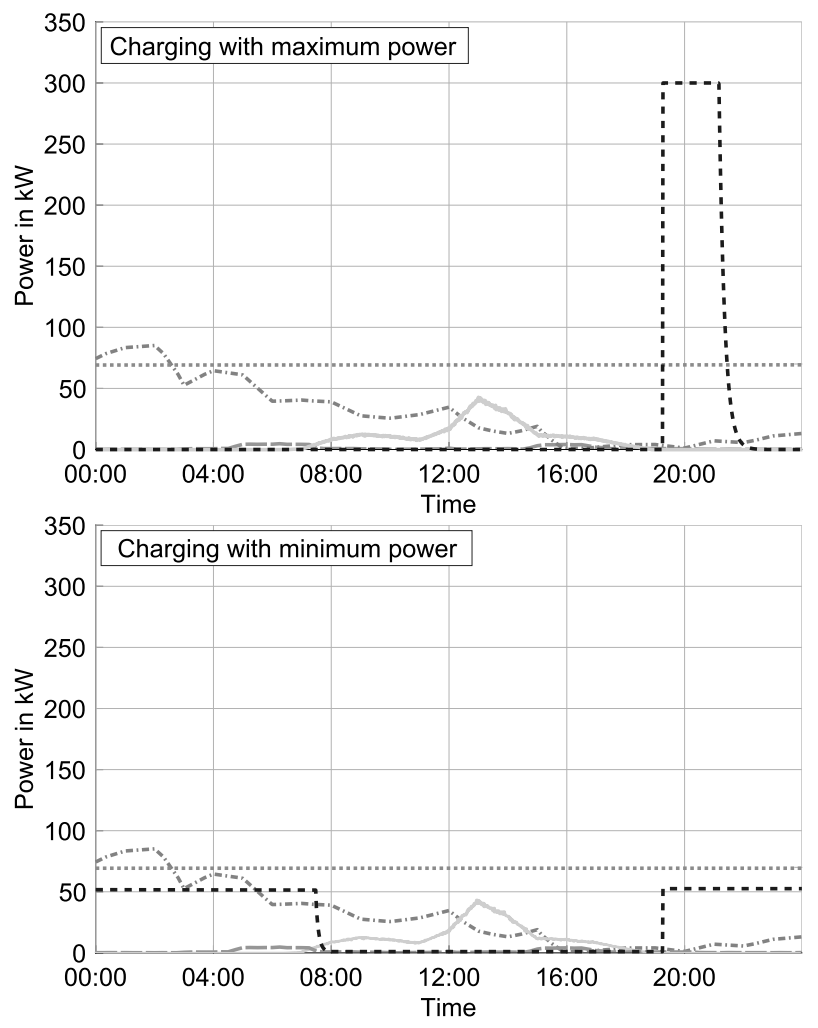

....... El. generation biogas - - - El. consumption charging station -El. generation PV - - El. consumption base load

$=-=$ El. generation wind

Figure 9: Electrical generation and consumption of different models on 15. September during sowing.

grid. In both scenarios with connection to the low voltage distribution grid, voltage band violations and equipment overloads occur on almost every power grid element, whereby the frequency of equipment overloads per year is slightly reduced by using the charging strategy Charging with minimum power compared to Charging with maximum power. Due to the limit value violations in the low voltage power grid and the maximum power grid supply of the individual agricultural operations, it can be useful to change the grid connection level of these operations to medium voltage. The medium voltage distribution grid did not show any limit value violations in the two scenarios with connection to the medium voltage distribution grid and is therefore suitable for agricultural operations with a previous connection to the low voltage distribution grid as a possible grid integration solution for all-electric agricultural machines. 


\section{Conclusion and Future Plans}

This paper describes preliminary studies for the research project "Concept and modeling of agricultural systems with renewable energy supply - Energy-4Agri". The development and evaluation of energy supply concepts for all-electric agricultural machines requires a holistic modelling of the involved technologies. This paper therefore provides information on the modelling and simulation of the technologies and energy systems.

Diesel consumption profiles were chosen as input data set for the all-electric agricultural machines. The conversion to electric power values can only be seen as a first approximation given a dynamic efficiency of combustion engines. Further research is therefore needed in the development of electric power profiles for all-electric agricultural machines, which take into account dynamic efficiency of the internal combustion engine and can thus provide more accurate simulation results. It has been shown that the charging strategy Charging with minimum power can already reduce the grid load in contrast to Charging with maximum power and increase the degree of self-sufficiency. In the future, further charging strategies can be modelled to determine the optimal charging times based on forecast data of the generation capacities of the renewable energies or depending on the power grid condition.

\section{Acknowledgement}

The work of this paper is originally based on the research project "Energy-4-Agri" (FKZ 03EI1013A). The authors acknowledge the support of the project within the energy research programme of the Federal Ministry of Economics and Energy and the Projektträger Jülich. The responsibility for the content of this publication lies with the authors and does not necessarily reflect the opinion of the project consortium Energy-4-Agri.

Supported by:

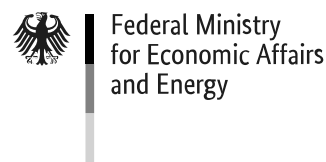

on the basis of a decision by the German Bundestag

\section{References}

[1] Frerichs L, Hanke S, Steinhaus S, Trösken L. EKoTech - A Holistic Approach to Reduce CO2 Emissions of Agricultural Machinery in Process Chains. In: 9th AVL International Commercial Powertrain Conference 2017, edited by SAE Mobilus, SAE Technical Paper Series. SAE International. 2017;

[2] Diekmann S, Reinhold C, Engel B. Centralized energy management for the optimization of residential districts. In: International ETG Congress 2017. ETG, Berlin and Offenbach: VDE Verlag GmbH. 2017; .

[3] Ortsgenaue Testreferenzjahre von Deutschland für mittlere, extreme und zukünftige Witterungsverhältnisse. Offenbach: Deutscher Wetterdienst and Bundesamt für Bauwesen und Raumordnung. 2017.

[4] Erichsen G. DWD weather model data for energy system simuation: 2017. 2020.

[5] NPS 100C-24: Datasheet. Northern Power Systems.

[6] Sonstige Marktregeln Strom: Kapitel 6: Zählwerte, Datenformate und standardisierte Lastprofile. Österreich: E-Control. 2015.

[7] Zimmermann R, Murillo-Sanchez C, Thomas R. MATPOWER: Steady-State Operations, Planning and Analysis Tool for Power Systems Research and Education. Power Systems, IEEE Transactions on. 2011;26(1):12-19.

[8] Reinhold C, Engel B. Simulation environment for investigations of energy flows in residential districts and energy management systems. In: International ETG Congress 2017. ETG, Berlin and Offenbach: VDE Verlag GmbH. 2017;

[9] Neiber J. Strombedarf und Eigenstromversorgung in der Nutztierhaltung. Mannheim. 2020.

[10] Kerber G. Aufnahmefaehigkeit von Niederspannungsverteilnetzen fuer die Einspeisung aus Photovoltaikkleinanlagen. Dissertation, Technische Universitaet Muenchen, Muenchen. 2010.

[11] Rudion K, Orths A, Styczynski ZA, Strunz K. Design of benchmark of medium voltage distribution network for investigation of DG integration. In: IEEE Power Engineering Society general meeting, 2006. IEEE, Canada: IEEE Operations Center. 2006; .

[12] Meier H, Fünfgeld C, Adam T, Schieferdecker B. Repräsentative VDEW-Lastprofile. Frankfurt am Main: VDEW. 1999.

[13] DKE Deutsche Kommission Elektrotechnik Elektronik Informationstechnik in DIN und VDE. Voltage characteristics of electricity supplied by public distribution networks. 50160. Berlin: Beuth Verlag GmbH. 2010. 


\title{
Orthotropic Non-hysteretic Permeability Model as a Lookup-table for FEA
}

\author{
Robert Courant*, Jürgen Maas \\ Mechatronic Systems Laboratory, Technische Universität Berlin, 10623 Berlin, Germany \\ *robert.courant@emk.tu-berlin.de
}

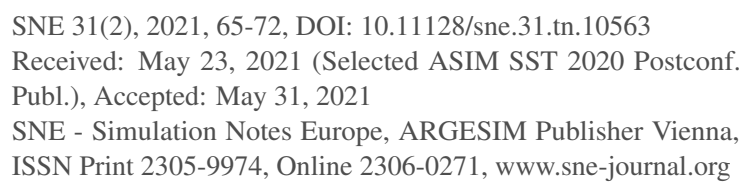

SNE - Simulation Notes Europe, ARGESIM Publisher Vienna, ISSN Print 2305-9974, Online 2306-0271, www.sne-journal.org

Abstract. Within this paper, we present an appropriate approach to prepare the data of orthotropic nonlinear soft magnetic materials for use in finite element analyses. Based on the co-energy density, an elliptic model for the interpolation between the principal directions is derived and extended to laminated materials. Instead of adapting the FE-code to incorporate the fundamental equations, a workflow is proposed to generate a lookup-table of the anisotropic nonlinear permeability which can be easily integrated in FE-programs like COMSOL Multiphysics. The modelled behaviour is shown for typical material classes and the lamination model is numerically validated.

\section{Introduction}

Soft magnetic materials are essential for almost all magnetic circuits. They play an important role in electromotors and transformers to guide the flux to guarantee an efficient operation[1],[2]. For the dimensioning of those circuits, accurate models of their magnetic behaviour are necessary. The most important characteristic is the relation between magnetic field strength $\mathbf{H}$ and magnetic flux density B. All ferromagnetic materials show a saturation at high fields, therefore a nonlinear approach has to be chosen. As the nonlinear problems are almost impossible to solve analytically for complex geometries, magnetic problems are typically computed numerically.

High performance soft magnetic materials often exhibit an anisotropic behaviour. Characteristic examples are different grain oriented (GO) electrical steels that show a magnetic easy axis in rolling direction and transverse magnetic hard axes. Consequently, magnetically anisotropic materials are typically characterised by uniaxial nonlinear curves $B_{\mathrm{u}, i}\left(H_{\mathrm{u}, i}\right)$ in three principal directions. This experimental characterisation of anisotropic materials requires sophisticated measurements[3],[4]. Because the flux components influence each other (even for isotropic materials), for vectors between the principal directions, a suitable interpolation has to be found. An extension of the topic are laminated materials, where non-ferromagnetic layers inhibit the perpendicular flux more than in plane.

This paper chooses an elliptical approach based on the co-energy density, which is well known concept[5],[6]. Key elements are based on the implementation of [7]. It has to be noted that hysteresis is not considered here. For magnetic materials, hysteresis is typically modelled with a JILES-ATHERTON model, other models like PREISACH or PRANDTL-ISHLINSKI are also applicable.

Another application of anisotropic magnetic properties, that attracted the author to the topic in the first place, are magnetic shape memory alloys, studied for their shape memory and magnetocaloric effect. These are indeed described by BH-curves in easy direction and in the perpendicular hard directions as shown in Figure 1. Therefore, they are appropriate benchmarking examples for our model.

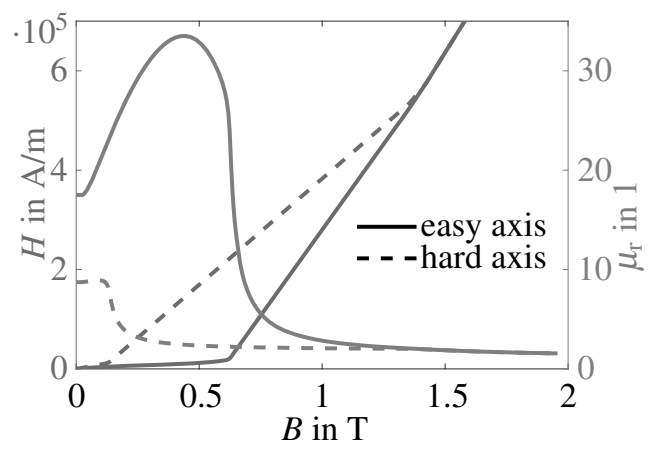

Figure 1: HB- and $\mu_{\mathrm{r}}(B)$-curves for easy and hard axis of examined MSMA from [8].

Section 1 introduces the physical background and motivates the elliptical model. This approach is gener- 
alized for nonlinear orthotropic materials in section 2 . Section 3 extends the model to laminated materials. Because magnetic anisotropy can be only incorporated as a relative permeability in the used FE-software COMSOL Multiphysics, the model is prepared as a look-up table of the nonlinear permeability dependent on the flux density B. In section 4, our model is analysed for different example materials. The laminate is numerically validated in section 5.

\section{Constitutive Equations}

Electromagnetic problems are described by MAXWELL's equations. There, two quantities each are defined for the electric and magnetic domain, one for the excitation and one for the effect. In the magnetic domain, these are the magnetic field strength $\mathbf{H}$ and the magnetic flux density $\mathbf{B}$. These two vector fields are coupled by generally nonlinear material properties

$$
\mathbf{B}=\mathbf{B}(\mathbf{H}) .
$$

The co-energy density of the magnetic field can be derived by

$$
w^{\prime}(\mathbf{B})=\int_{0}^{\mathbf{B}} \mathbf{H} \cdot \mathrm{d} \tilde{\mathbf{B}} .
$$

In the easiest case of vacuum this is

$$
w^{\prime}(\mathbf{B})=\frac{\mathbf{B}^{2}}{2 \mu_{0}}
$$

which leads to the linear coupling of $\mathbf{B}$ and $\mathbf{H}$

$$
\mathbf{B}=\mu_{0} \mathbf{H}
$$

For general linear materials, this is extended using Einstein notation to

$$
B_{j}=\mu_{0} \mu_{\mathrm{r}, i j} H_{i}
$$

with the relative permeability matrix $\mu_{\mathrm{r}, i j}$. In most cases, the relative permeability is assumed orthotropic and the matrix is thus

$$
\frac{1}{\mu_{\mathrm{r}, i j}}=\left(\begin{array}{ccc}
\frac{1}{\mu_{\mathrm{r}, x}} & 0 & 0 \\
0 & \frac{1}{\mu_{\mathrm{r}, y}} & 0 \\
0 & 0 & \frac{1}{\mu_{\mathrm{r}, z}}
\end{array}\right) .
$$
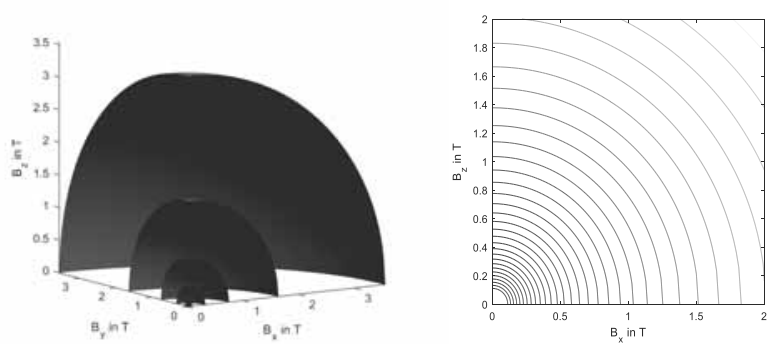

Figure 2: Contour lines of the magnetic co-energy for a linear isotropic material with $\mu_{\mathrm{r}, \text { iso }}=5000$.
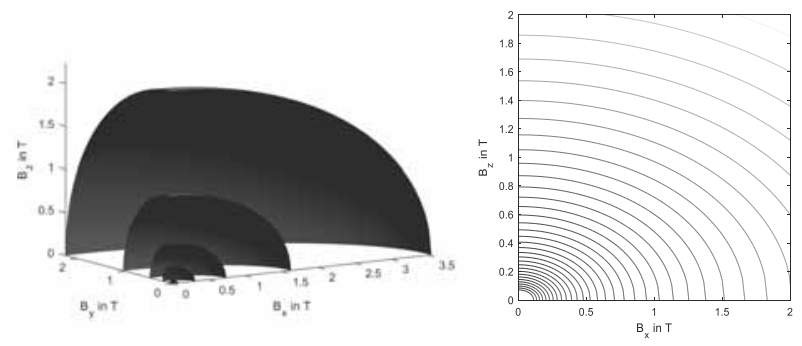

Figure 3: Contour lines of the magnetic co-energy for a linear anisotropic material.

The co-energy density for this case is

$$
\begin{aligned}
w^{\prime}(\mathbf{B}) & =\int_{0}^{\mathbf{B}} \frac{1}{\mu_{0} \mu_{\mathrm{r}, i j}} \tilde{B}_{j} \cdot \mathrm{d} \tilde{B}_{i} \\
& =\frac{B_{x}^{2}}{2 \mu_{0} \mu_{\mathrm{r}, x}}+\frac{B_{y}^{2}}{2 \mu_{0} \mu_{\mathrm{r}, y}}+\frac{B_{z}^{2}}{2 \mu_{0} \mu_{\mathrm{r}, z}} .
\end{aligned}
$$

The contour surfaces $w^{\prime}=$ const. of this are concentric ellipsoids. This is why an elliptic model is used in section 4 to model anisotropic nonlinear functions. Figure 2 depicts the contour surfaces in $3 \mathrm{D}$ or contour lines in $2 \mathrm{D}$ for a linear isotropic material with $\mu_{\mathrm{r} \text {,iso }}=5000$. The contour surfaces are concentric spheres. Figure 3 shows the expected ellipsoids for a linear anisotropic material with $\mu_{\mathrm{r}, x}=5000$ and $\mu_{\mathrm{r}, y}=\mu_{\mathrm{r}, z}=2000$.

Nonlinear isotropic materials have equally oriented field and flux, where the absolute value is described by a nonlinear relation as

$$
\mathbf{B}=B_{\text {iso }}(|\mathbf{H}|) \frac{\mathbf{H}}{|\mathbf{H}|} .
$$

The use of the absolute value produces a spherical interpolation between the directions. Although the shown relation $\mathbf{B}(\mathbf{H})$ is the more comprehensible, for numerical 
validations the opposite formulation $\mathbf{H}(\mathbf{B})$ is preferable, because in most FE-programs, the problem is solved using the auxiliary vector potential $\mathbf{A}$ that is linked to the flux density by $\mathbf{B}=\operatorname{rot}(\mathbf{A})$. Accordingly, in section 3 we will setup the lookup-table for the nonlinear function of $\mu_{\mathrm{r}}$ dependent on $\mathbf{B}$ in the form of

$$
\left(B_{x}\left|B_{y}\right| B_{z}\left|\mu_{\mathrm{r}, x}\right| \mu_{\mathrm{r}, y} \mid \mu_{\mathrm{r}, z}\right) .
$$

\section{Elliptic Interpolation}

The interpolation for nonlinear orthotropic materials has to fulfil three conditions. Firstly, it has to be a convex function, which translates to convex contour lines in $2 \mathrm{D}$ or surfaces in $3 \mathrm{D}$. Secondly, the interpolation has to match the uniaxial nonlinear curves it is fitted to. Finally, the approach has to match the elliptical model of the co-energy density as shown in (7) for linear orthotropic magnetic materials. An obvious choice, that meets the requirements, is again an elliptical model of the co-energy density $w^{\prime}(\mathbf{B})$, where the half-axes in all directions of the contour surface for each co-energy density $w^{\prime}$ are determined by the corresponding flux density $B_{\mathrm{u}, i}\left(w^{\prime}\right)$ of the uniaxial measurements.

These measurements are typically provided as a table $\left(H_{\mathrm{u}, i} \mid B_{\mathrm{u}, i}\right)$. The co-energy densities can be numerically integrated according to (2). From the extended table $\left(H_{\mathrm{u}, i}\left|B_{\mathrm{u}, i}\right| w^{\prime}\right)$ a function $B_{\mathrm{u}, i}\left(w^{\prime}\right)$ can be interpolated. For each $w^{\prime}$, the contour surface is described by

$$
\left(\frac{B_{x}}{B_{\mathrm{u}, x}\left(w^{\prime}\right)}\right)^{2}+\left(\frac{B_{y}}{B_{\mathrm{u}, y}\left(w^{\prime}\right)}\right)^{2}+\left(\frac{B_{z}}{B_{\mathrm{u}, z}\left(w^{\prime}\right)}\right)^{2}=1 .
$$

Figure 4 illustrates the two-dimensional case with a contour line. The interpolation scheme is to generate preferably equally spaced sample points of the ellipsoids for each co-energy density $\left(B_{x}\left|B_{y}\right| B_{z} \mid w^{\prime}\right)$. These values can be interpolated to a function $w^{\prime}\left(B_{x}, B_{y}, B_{z}\right)$.

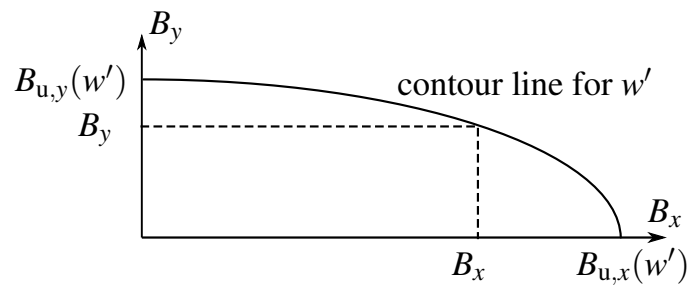

Figure 4: 2D representation of the elliptic interpolation.

\section{Lamination}

Laminated magnetic materials could be modelled with their full geometry as shown in section 5. Due to the thin layers, especially of the non-magnetic insulation, an otherwise unnecessarily fine mesh is required which increases the computational burden. Therefore, for most applications a homogenised material model suffices. The insulation material 2 is non-ferromagnetic $\mu_{\mathrm{r}}=1$. The stacking factor $f$ is the volume ration of the ferromagnetic material 1 to the overall volume. The sheets are thin, a change in the field from one sheet to the next can be neglected. Without loss of generality, the sheets are assumed to be in the xy-plane and stacked in $\mathrm{z}$ direction. At interfaces, the normal component of the flux density is continuous, therefore the homogenized flux density is

$$
B_{z}=B_{1, z}=B_{2, z}
$$

The homogenized flux density in the xy-plane is constructed by a mixing law

$$
\begin{aligned}
& B_{x}=f B_{1, x}+(1-f) B_{2, x} \\
& B_{y}=f B_{1, y}+(1-f) B_{2, y} .
\end{aligned}
$$

The homogenized co-energy density $w_{\text {lam }}^{\prime}$ can be calculated as a weighted sum of that of the ferromagnetic material $w_{1}^{\prime}\left(B_{1, x}, B_{1, y}, B_{1, z}\right)$ from the interpolation and that of vacuum from (3) and is dependent on the homogenized flux density and that in the insulation

$$
\begin{aligned}
& w_{\text {lam }}^{\prime}\left(B_{x}, B_{y}, B_{z}, B_{2, x}, B_{2, y}\right) \\
& =f w_{1}^{\prime}\left(\frac{1}{f}\left(B_{x}-(1-f) B_{2, x}\right), \frac{1}{f}\left(B_{y}-(1-f) B_{2, y}\right), B_{z}\right) \\
& +\frac{1-f}{2 \mu_{0}}\left(B_{2, x}^{2}+B_{2, y}^{2}+B_{z}^{2}\right) .
\end{aligned}
$$

For a strong ferromagnetic material $1, B_{2, x}$ and $B_{2, y}$ can be neglected and the approximated co-energy density is

$$
w_{\text {lam }}^{\prime}(\mathbf{B})=f w_{1}^{\prime}\left(\frac{B_{x}}{f}, \frac{B_{y}}{f}, B_{z}\right)+\frac{1-f}{2 \mu_{0}} B_{z}^{2} .
$$

Relative permeability. The relative permeabilities for the look-up table (9) could be calculated using (5) with the field strength $\mathbf{H}$ derived according to (2) as

$$
\mathbf{H}_{\mathrm{lam}}(\mathbf{B})=\frac{\partial w_{\mathrm{lam}}^{\prime}(\mathbf{B})}{\partial \mathbf{B}} .
$$


While this is physically correct, the numerical derivate on the interpolated function $w_{\text {lam }}^{\prime}(\mathbf{B})$ is not very accurate for a realistic sampling grid. Instead, the permeability can be computed from the uniaxial measurements $\left(H_{\mathrm{u}, i} \mid B_{\mathrm{u}, i}\right)$. Using the analytical derivate of (14), the uniaxial field strengths of the complete laminated material can be calculated as

$$
\begin{aligned}
& H_{\mathrm{lam}, \mathrm{u}, x}\left(B_{\mathrm{u}, x}\right)=H_{\mathrm{u}, x}\left(\frac{B_{\mathrm{u}, x}}{f}\right) \\
& H_{\mathrm{lam}, \mathrm{u}, y}\left(B_{\mathrm{u}, y}\right)=H_{\mathrm{u}, y}\left(\frac{B_{\mathrm{u}, y}}{f}\right) \\
& H_{\mathrm{lam}, \mathrm{u}, z}\left(B_{\mathrm{u}, z}\right)=f H_{\mathrm{u}, z}\left(B_{\mathrm{u}, z}\right)+\frac{1-f}{\mu_{0}} B_{\mathrm{u}, z} .
\end{aligned}
$$

Now, (5) can be solved for $\mu_{\mathrm{r}, \mathrm{lam}, i}\left(B_{\mathrm{u}, z}\right)$. For the uniaxial values, the ferromagnetic material's contribution to the co-energy density

$$
w_{\text {an }}^{\prime}=w_{1}^{\prime}\left(\frac{B_{x}}{f}, \frac{B_{y}}{f}, B_{z}\right)
$$

can be accurately integrated according to (2). While the overall behaviour of the laminate is dependent on the complete co-energy density $w_{\text {lam }}^{\prime}$, the relative permeabilities of the ferromagnetic material are only dependent on the new co-energy density $w_{\text {an }}^{\prime}$. Therefore, the permeabilities of the ferromagnetic material and consequently of the laminate can be derived from the uniaxial measurements. Consequently, the generation of the look-up table (9) consists of two steps: First, $w_{\text {an }}^{\prime}\left(B_{x}, B_{y}, B_{z}\right)$ is calculated on a grid $\left(B_{x}\left|B_{y}\right| B_{z}\right)$, then the permeabilities $\left(\mu_{\mathrm{r}, \mathrm{lam}, x}\left|\mu_{\mathrm{r}, \mathrm{lam}, y}\right| \mu_{\mathrm{r}, \mathrm{lam}, z}\right)$ are taken from the uniaxial measurement with the corresponding co-energy density $w_{\text {an }}^{\prime}$. The presented approach yields a far smoother permeability than the direct derivation according to (15), especially at low flux densities.

\section{Application}

Applying our method to the example depicted in Figure 3 shows no difference. Therefore, the third condition is fulfilled. The second condition can be tested by comparing $\mu_{\mathrm{r}}$ along an axis from the elliptic model with the uniaxial input data. The interpolation shall be applied to a representative $\mathrm{GO}$ electrical steel of grade M-6. The $\mathrm{BH}$-curve is taken from the COMSOL material library, the results are shown in Figure 5. The rolling direction has a much higher initial permeability, proportional to the derivate of the curve, than the transverse direction.

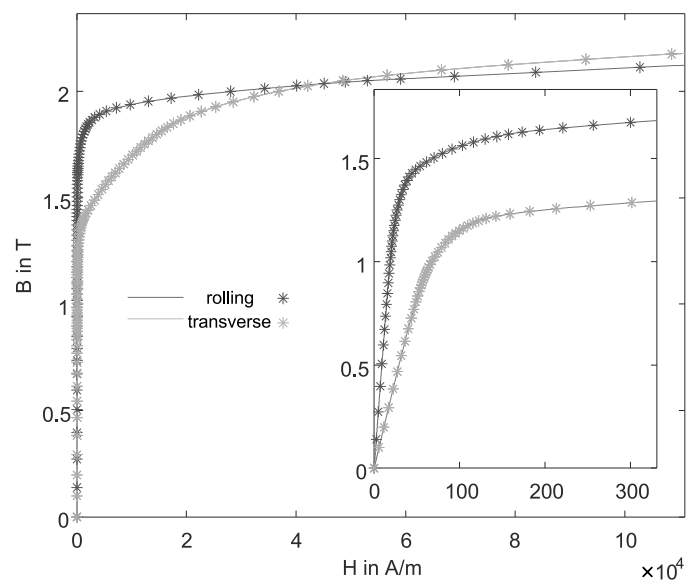

Figure 5: $\mathrm{BH}$-curve of the investigated grain oriented steel M-6 in both principal directions, detail on the right.
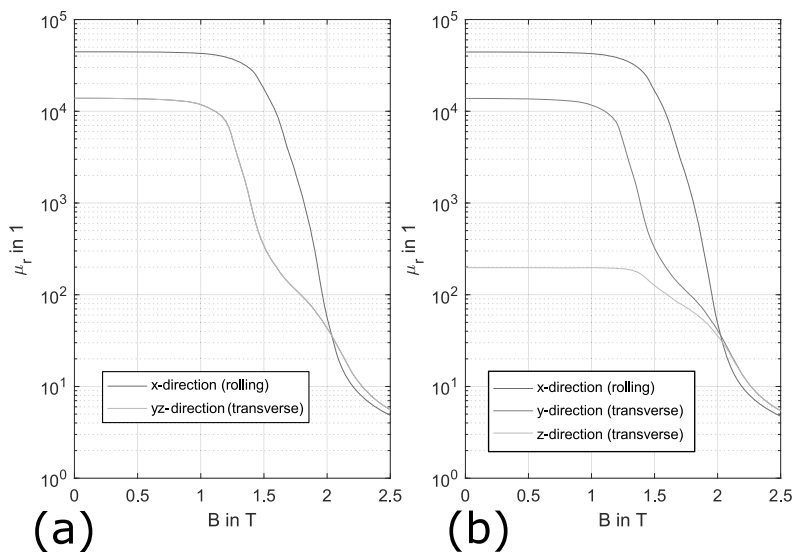

Figure 6: $\mu_{\mathrm{r}, i}\left(B_{\mathrm{r}, i}\right)$ in all directions, (a) of the investigated GO steel M-6 and (b) of the laminate with stacking factor $f=0.995$.

At very high fields, the transverse direction gains a slight advantage. This can also be seen in Figure 6 (a) of the elliptic model for this material with the rolling direction in $x$ and the transverse curves used for $y$ and $z$. The curves of the uniaxial measurements perfectly match the model, therefore the second condition is fulfilled.

The contour surfaces or contour lines in a 2D-section for the elliptical model of this material can be seen in Figure 7. At lower fields, the difference between the axes is higher, almost reaching a factor of two, while the results approximately match at higher fields in accordance with the input curves. The density of the contour lines in the $2 \mathrm{D}$-section is correlated with the permeability. At low fields, the permeability is high and the energy doesn't 

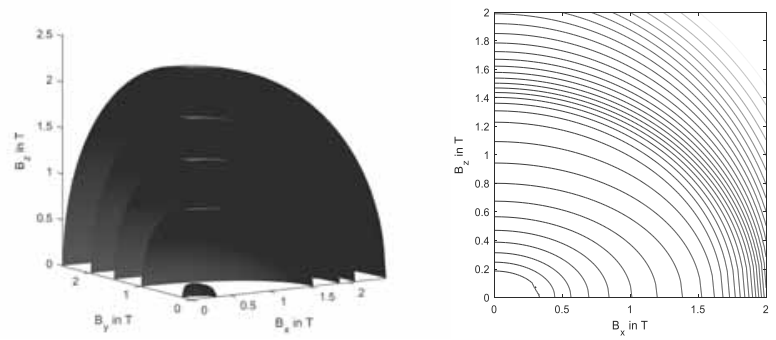

Figure 7: Contour lines of the magnetic co-energy density for pure grain oriented steel without lamination.
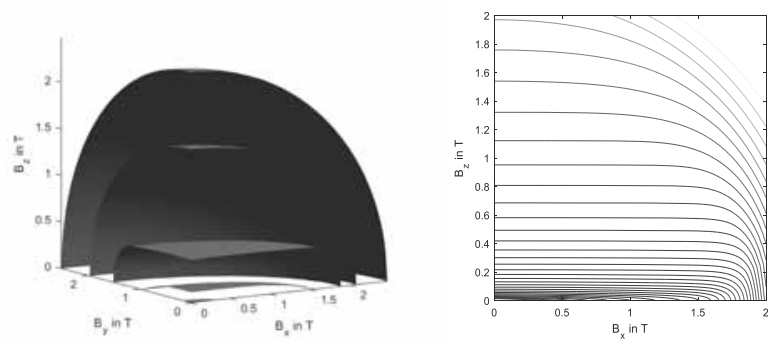

Figure 8: Contour lines of the magnetic co-energy density for laminated grain oriented steel with $f=0.995$.

grow that fast, after the saturation, more energy is needed to still increase the field. When the axes roughly match for high fields, the contour lines are almost concentric circles.

In Figure 8 and Figure 9, laminates of the same grain oriented steel with different stacking factors are shown. In Figure 8, the stacking factor is $f=0.995$, meaning that only $0.5 \%$ are non-magnetic insulation. Nevertheless, for low co-energy densities the flux in z-direction changed drastically. The former ellipses in 2D and ellipsoids in 3D are flattened with an almost parallel top.
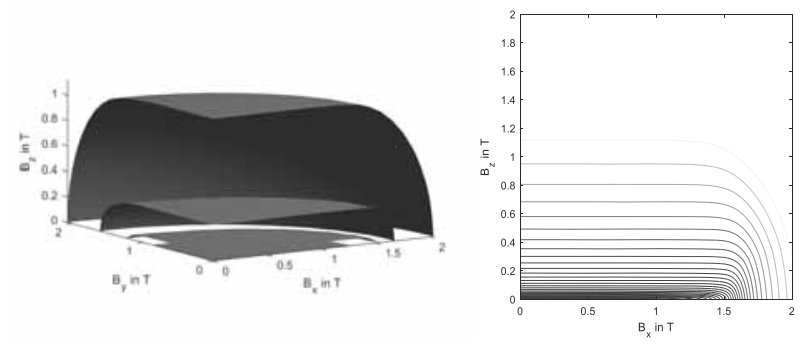

Figure 9: Contour lines of the magnetic co-energy density for laminated grain oriented steel with $f=0.8$.
The corresponding relative permeabilities can be seen in Figure 6 (b). Because of the almost identical cross section compared to the full GO material, the in-plane curves $x$ and $y$ are also almost identical between (a) and (b). In contrast to that relative permeability in stacking direction $z$ is greatly reduced. With increasing saturation, the effect of the gap becomes less prominent, until both transverse directions are almost identical again. The contour surfaces or contour lines of the ferromagnetic material's contribution to the co-energy density $w_{\text {an }}^{\prime}$ according to (17) are almost indistinguishable from the co-energy density of the pure GO steel without lamination in Figure 7 and are therefore not separately shown. This is again because of the almost identical cross section.

The effect extends to higher co-energy densities with the lower stacking factor $f=0.8$ in Figure 9. The field in $z$ is dominated by the insulation even for higher fields where the ferromagnetic materials begins to reach the saturation. With the reduced cross section area of the ferromagnetic material, the flux in $x$ and $y$ is also slightly reduced and the saturation is reached a bit earlier.

Another example to test our model are magnetic shape memory alloys (MSMA). Figure 1 shows the characteristic HB-curves for the easy and hard axis. The results of the elliptic model are depicted in Figure 10. While the initial permeability of the GO steel was around 40000 in rolling direction, the MSMA only reaches 30. Therefore, higher co-energy densities are evaluated to cover the same flux density range. The MSMA's anisotropy is larger than that of the GO steel. The change in permeability isn't as high, consequently the contour lines in 2D are more equally spaced.
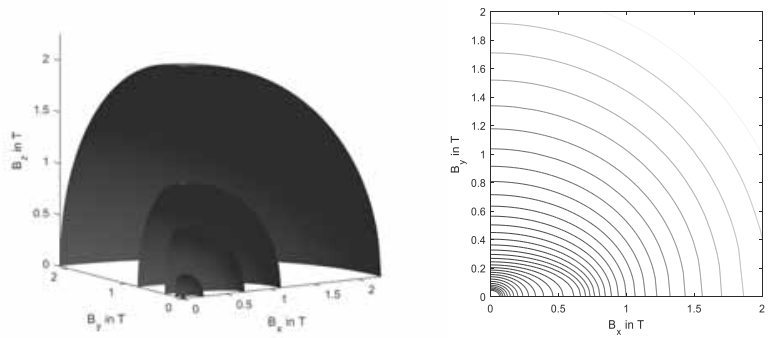

Figure 10: Contour lines of the magnetic co-energy density for MSMA with easy axis in $x$. 


\section{Numerical Validation}

To validate the laminated model, a benchmark FEsimulation is conducted in COMSOL, comparing the geometrically modelled laminate with an averaged continuum using the generated look-up table (9) at different angles with $\varphi=0^{\circ}$ denoting the flux in stacking direction $z$ and $\varphi=9^{\circ}$ ! for the flux in rolling direction $x$. For a decent accuracy, a table with 200 flux densities in each direction is used. As described in the introduction, magnetically anisotropic materials have to be implemented as relative permeabilities dependent on the flux density B. In contrast to that, the materials from the included material library are implemented as isotropic BH-curves as shown in Figure 5. To exclude errors in the developed anisotropic model, the comparison is therefore conducted with the isotropic material of GO M-6 in rolling direction from the same diagram. For the laminate, a stacking factor $f=0.95$ is investigated.

Additionally the relative permeability of the laminate rotated by $\varphi$ is calculated analytically. The co-energy density $w_{\text {an }}^{\prime}$ in the ferromagnetic material is

$$
w_{\text {an }}^{\prime}(B, \varphi)=w_{\text {an }}^{\prime}(B \sin (\varphi), 0, B \cos (\varphi)) .
$$

Using the look-up table, the relative permeabilities in material coordinates $\mu_{\mathrm{r}, i, \text { mat }}$ can be determined. Using those, the magnetic fields in material coordinates are

$$
\begin{aligned}
H_{x, \text { mat }}(B, \varphi) & =\frac{B \sin (\varphi)}{\mu_{\mathrm{r}, x, \text { mat }}\left(w_{\text {an }}^{\prime}(B, \varphi)\right) \cdot \mu_{0}} \\
H_{z, \text { mat }}(B, \varphi) & =\frac{B \cos (\varphi)}{\mu_{\mathrm{r}, z, \text { mat }}\left(w_{\text {an }}^{\prime}(B, \varphi)\right) \cdot \mu_{0}}
\end{aligned}
$$

The analytical field in flux direction and the relative permeability are then

$$
\begin{aligned}
H_{\mathrm{ana}}(B, \varphi) & =\sin (\varphi) H_{x, \mathrm{mat}}+\cos (\varphi) H_{z, \mathrm{mat}} \\
\mu_{\mathrm{r}, \mathrm{ana}}(B, \varphi) & =\frac{B}{H_{\mathrm{ana}} \cdot \mu_{0}}
\end{aligned}
$$

The model setup in COMSOL is shown in Figure 11. It consists of four tests, evaluated in the domain between the orange lines and connected parallelly to the same excitation. On the left, a material using the look-uptable of the laminated material is defined (magenta). To measure the material response at different angles, the material coordinates are rotated accordingly. This test of the averaged lamination model is designated "avg". The other three tests are different implementations of the

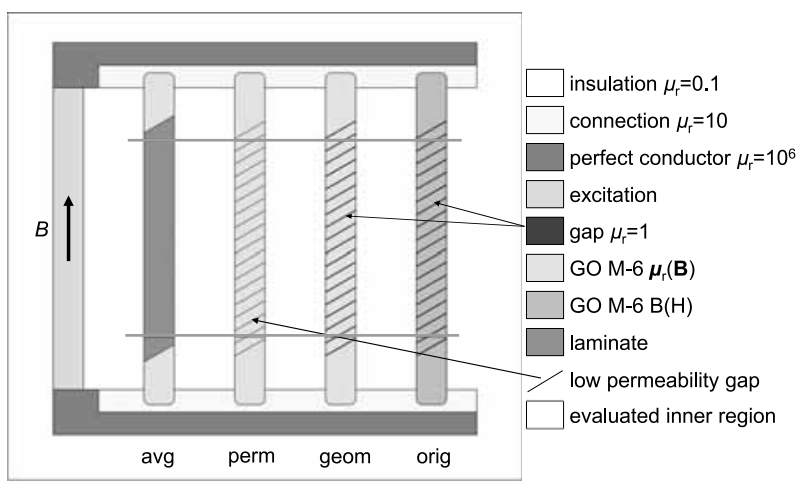

Figure 11: Model setup for rotation angle $\varphi=30^{\circ}$ with used materials and test designation at the bottom.

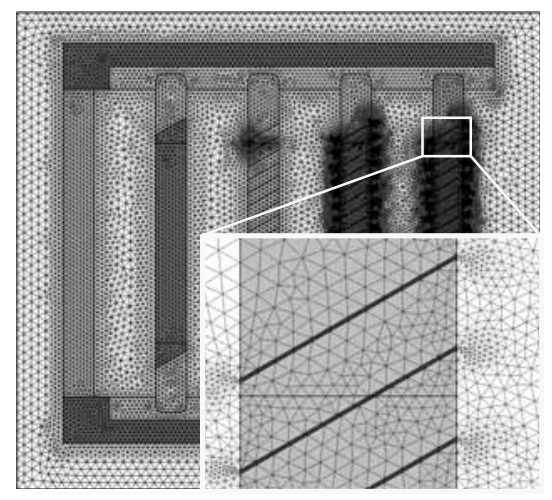

Figure 12: Used mesh, detail of the fully modelled laminate with two elements over the gap.

complete stacked geometry, which is again rotated for different cases.

The test designated "perm" uses a so-called "low permeability gap" between the layers of GO M-6. This special transition condition is used to model a thin gap of non-ferromagnetic material on a boundary instead of in a domain. An advantage is the easier mesh without the thin domains, the internal behaviour is not automatically calculated though. While the magnetic flux is computed correctly, the field strength and energy in the gap have to be considered manually to calculate the average permeability of the laminate.

The last two tests designated "geom" and "orig" use the fully modelled gaps. This can be seen in the detail of Figure 12, where the thin blue gap with $\mu_{\mathrm{r}}=1$ is shown. The gaps are identical for both tests, but the isotropic GO material is modelled differently. While for "geom" the look-up table is used, "orig" is modelled direly using the aforementioned BH-curve from Figure 5. 


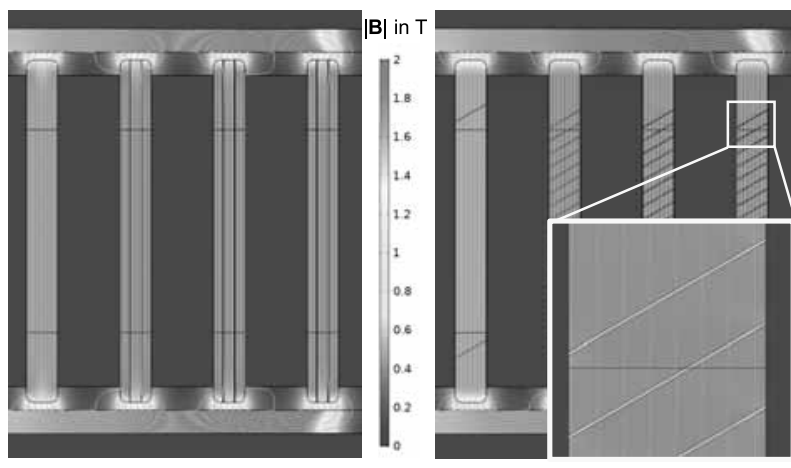

Figure 13: Resulting fields for an excitation of 7T, orientation of the laminate left $\varphi=90^{\circ}$, right $\varphi=3^{\circ}$ !, fluxlines in light grey.

The excitation has to be connected to the angled tests in a numerically stable way for a wide flux range. This is done in three steps. First, a vertical remanent flux is defined in the green excitation domain, this flux is distributed through the red almost perfect conductor with $\mu_{\mathrm{r}}=10^{6}$. To avoid steep gradients at the interface to the laminated material especially at $\varphi=90^{\circ}$ (see Figure 13 on the left), a connection layer with a lower permeability of $\mu_{\mathrm{r}}=10$ is defined and the corners are rounded. After a short layer of the non-laminated GO material, the test materials start outside the evaluated inner region. The necessary air region around the flux guiding geometries has a reduced permeability of $\mu_{\mathrm{r}}=0.1$. While this magnetic insulation is unphysical, it helps to concentrate the flux inside the test domains even at high saturation. Without this addition, the flux inside the test domains would be less uniformly vertical. We can therefore be even more accurate at high flux densities than physical experiments with the same geometry could be. The mesh can be seen in Figure 12. While a sufficiently fine mesh is defined for most domains, special care is required at the thin gaps in "geom" and "orig". These are done with a mapped mesh with two elements over the gap as shown in the detail view.

The local flux densities for two load cases can be seen in Figure 13. Both show an excitation of 7T which results in approximately $1.8 \mathrm{~T}$ in the test domains. Therefore, the GO steel is already in the beginning saturation according to Figure 6. While the overall flux is oriented vertically in the test domains in all cases, the detail at $\varphi=30^{\circ}$ shows a zig-zag pattern of the (grey) flux lines between the GO regions and the gaps. Therefore, the bridged length of the gap is reduced and accordingly

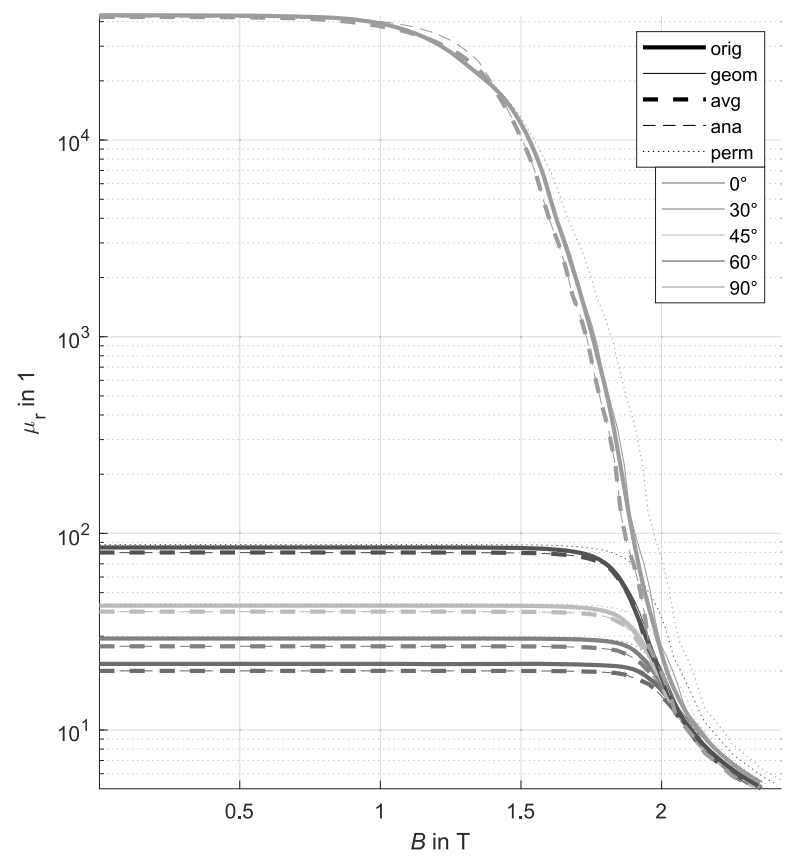

Figure 14: Resulting relative permeability $\mu_{\mathrm{r}}(B)$ for the specified tests (line styles) and angles (line colours).

the magnetic resistance. A much thinner, almost onedimensional geometry, would reduce this effect. In turn a sleek test region would suffer more flux leakage through the adjacent air regions.

The reduced magnetic resistance of the geometrically modelled tests "geom" and "orig" for diagonal layers compared to the averaged model can be also seen in the resulting relative permeabilities in Figure 14. There, the angles are colour coded and the four test scenarios and the analytic calculation according to (20) are denoted with different line styles. The two geometrically modelled tests "geom" and "orig" are shown with full lines, with a thicker line for "orig", the implementation using the original material model. Apart from some ripples in the saturation region a complete fit can be observed. Those ripples are caused by the interpolation on the look-up table and dependent on its resolution.

The simulated averaged model is marked with a thick dashed line. The thin dashed line denotes the test "ana". This is the analytical calculation according to (20) applied to the average flux density in the test domain. Apart from a slight deviation at the beginning saturation at $\varphi=90^{\circ}$, the analytical calculation "ana" and the implemented model "avg" are a complete match. The ripples in both curves are again caused by the resolution of the 
look-up table. The dotted line denoting the test "perm" with the low permeability gap overestimates the permeability at higher flux densities in rolling direction (higher angles towards $\varphi=90^{\circ}$ ), because the reduced cross section of the ferromagnetic material cannot be taken into account in this setup. This deviation would increase for higher stacking factors.

The overall performance of the developed averaged model can be evaluated comparing the thick solid line for the original model and the thick dashed line for the averaged model. The saturation behaviour is replicated accurately for all angles. The starting permeabilities meet exactly the analytical expectation, while the original model slightly overestimates them for a flux in stacking direction (lower angles towards $\varphi=0^{\circ}$ ), most likely caused by the discretization in the thin gaps.

\section{Conclusion}

The proposed elliptic interpolation of the co-energy density produces convex functions for a stable and thermodynamically consistent simulation. The generated look-up table of the relative permeabilities $\mu_{\mathrm{r}, i}$ can be easily imported in FE-software like COMSOL Multiphysics. While we showed the interpolation between perpendicular uniaxial measurements, the data of other directions could be also included in the construction of the contour surfaces. This might be particular useful for GO steel at low fields where the angle between the magnetic hard direction and the easy rolling direction is close to $60^{\circ}$ as discussed in [9]. Our model can be applied to anisotropic materials with totally different permeability ranges as shown for GO steel and MSMA.

For laminated materials, with an increasing stacking factor the lamination becomes dominant compared to the anisotropy of the base ferromagnetic material. The overall behaviour of the laminate is dependent on the complete co-energy density $w_{\text {lam }}^{\prime}$, in contrast to that, the permeability is dependent on the co-energy density $w_{\text {an }}^{\prime}$ in the ferromagnetic material. Even very high stacking factors $f$ with only small gaps in the ferromagnetic material already cause a drastic change in the contour lines and surfaces of $w_{\text {lam }}^{\prime}$ compared to the material without lamination, while $w_{\text {an }}^{\prime}$ is less effected. The permeabilities calculated from $w_{\text {an }}^{\prime}$ are much smoother than those generated from the gradient of $w_{\text {lam }}^{\prime}$ and correctly replicate the high anisotropy.

The numerical validation shows a very high accordance of the fully modelled laminate and the deduced homogenization rule. This opens many possibilities for efficient models of magnetic circuits without the need to mesh the thin gaps. For dynamic simulations, the described magnetic behaviour is not sufficient. Besides hysteresis, the electrical conductivity $\sigma$ becomes important to estimate eddy currents. For laminated stacks, different models of $\sigma$ are compared in[10].

\section{References}

[1] S. Lopez, et al. Grain Oriented Steel Assembly Characterization for the Development of High Efficiency AC Rotating Electrical Machines, IEEE Trans Magn. 2009; 45(10): 4161-4164. doi: 10.1109/TMAG.2009.2023243

[2] Y. Ushigami, et al. Recent development of low-loss grain-oriented silicon steel, J Magn Magn Mater. 2003; 254-255: 307-314. doi: 10.1016/S0304-8853(02)00933-2

[3] T. Nakata, et al. Measurement of magnetic characteristics along arbitrary directions of grain-oriented silicon steel up to high flux densities, IEEE Trans Magn. 1993; 29(6): 3544-3546. doi: $10.1109 / 20.281224$

[4] D. Miyagi, et al. Development of Measuring Equipment of DC-Biased Magnetic Properties Using Open-Type Single-Sheet Tester, IEEE Trans Magn. 2006; 42(10): 2846-2848. doi: 10.1109/TMAG.2006.879144

[5] A. Di Napoli, R. Paggi. A model of anisotropic grain-oriented steel, IEEE Trans Magn. 1983; 19(4): 1557-1561. doi: 10.1109/TMAG.1983.1062582

[6] J.C. Sabonnadiere, G. Meunier. Modelling Nonlinear Magnetic Materials for Field Computation, J ELECTROMAGNET WAVE. 1990; 4(11): 1027-1054. doi: 10.1163/156939390X00357

[7] J. Krause, Energy conserving Anisotropic Anhysteretic Magnetic Modelling for Finite Element Analysis. 2012; arXiv:1212.5163v1 [math-ph]

[8] M. Schautzgy, U. Kosiedowski, T. Schiepp. 3D-FEM-Simulation of Magnetic Shape Memory Actuators, Proc. of 2016 COMSOL Conf.; 2016 Oct; Munich.

[9] H. Wang, C.-S. Li, T. Zhu. Hard magnetization direction and its relation with magnetic permeability of highly grain-oriented electrical steel, INT J MIN MET MATER. 2014; 21(11): 1077-1082. doi: $10.1007 / \mathrm{s} 12613-014-1012-8$

[10] J. Ziske, H. Neubert R. Disselnkötter. Modeling of Anisotropic Laminated Magnetic Cores using Homogenization Approaches, Proc. of 2014 COMSOL Conf.; 2014 Sep; Cambridge. 


\title{
NSA-DEVS: Combining Mealy Behaviour and Causality
}

\author{
Peter Junglas* \\ Dep. of Engineering “Dr. Jürgen Ulderup”, PHWT Vechta/Diepholz, Schlesierstr. 13a, 49356 Diepholz, Germany; \\ *peter@peter-junglas.de
}

SNE 31(2), 2021, 73-80, DOI: 10.11128/sne.31.tn.10564 Received: January 20, 2021 (Selected ASIM SST 2020 Postconf. Publ.), Revised May 24, 2021; Accepted: May 31, 2021

SNE - Simulation Notes Europe, ARGESIM Publisher Vienna, ISSN Print 2305-9974, Online 2306-0271, www.sne-journal.org

Abstract. The RPDEVS ("Revised PDEVS") formalism has been introduced to allow for a simple description of Mealy-type components that behave consistently. This made it necessary to change the way the simulator handles event chains. Using a simple example model we show that the proposed algorithm has serious problems with the resulting sequence of concurrent events. Therefore we introduce NSA-DEVS, a variant formalism that is inspired by ideas from non-standard analysis (NSA). It uses infinitesimal time delays to make a natural ordering of concurrent events easy, without the need to fix lots of additional parameters. As proof of concept we describe the example model in NSA-DEVS and implement it in a suitably twisted RPDEVS simulator.

\section{Introduction}

More than 40 years after its invention the DEVS formalism [1] and its most popular variant PDEVS [2] are now standard tools for the mathematical modeling of discrete-event systems. If in doubt a quick search for "DEVS formalism" in Google scholar reveals over 6000 papers and shows that the seminal book of Zeigler et al. [3] has been cited about 7000 times.

Looking at widely-used simulation environments, the picture changes completely: Neither Arena [4] nor SimEvents [5] use DEVS internally or even mention it in their documentation. And though Mathworks has based its redesign of SimEvents on a proper modeling formalism, the developers didn't choose DEVS for this purpose [6].
On the other hand there are quite a few free simulation programs available that use DEVS or one of its variants for the definition of atomic components and the implementation of coupled systems [7]. But all of them twist the original DEVS formalism to make it a suitable foundation for a concrete simulation environment [8]. Some of the problems are just minor nuisances, like the addition of input and output ports, others are of a more fundamental nature.

Probably the most serious flaw has been named by Preyser et al., who show in [9], that PDEVS has difficulties modeling certain Mealy-type components: The necessary introduction of transitional states leads to delays that change the expected order of concurrent events and the behaviour of subsequent components. This is a serious drawback, if one wants to define a library of reusable blocks. Therefore the PDEVS formalism has been altered in [10] to allow for Mealy-like behaviour thereby introducing the revised version RPDEVS.

To make this work, one has to change the way chains of concurrent events are handled, which is a complex and possibly dangerous endeavour. Even after the careful analysis in [10] and the formal definition of an RPDEVS simulator [11] the question remains, whether the proposed scheme is capable of handling the subtle problems that appear in practical modeling tasks.

To further investigate the status of RPDEVS we will introduce a simple example that is plagued by a complex causal structure of concurrent events, and implement it in PDEVS and RPDEVS, using freely available simulators.

Since the results show that RPDEVS has problems with the example model, we will propose a different way of how to cope with concurrent event chains, which uses concepts of non-standard analysis [12]. After a short introduction to the basic mathematical ideas, we will define the new DEVS variant NSA-DEVS, which combines the ideas of RPDEVS with a more robust method to handle concurrent events. 
Finally we will implement the standard example in NSA-DEVS and find that it can handle this model in a clear-cut, easily understandable way. This supports the assumption that NSA-DEVS might be a good basis for concrete modeling and simulation environments, since it combines the security in the handling of concurrent events from PDEVS with the modeling power of RPDEVS.

\section{Singleserver - a Fundamental Example}

The singleserver example used in the following consists of a generator that creates entities in fixed time intervals $t_{G}=1$ and sends them to a queue, which is connected to a simple server with fixed service time $t_{S}=1.5$. Entities leaving the server are terminated (cf. Fig. 1). Additionally the queue and the server output the current number of entities stored.

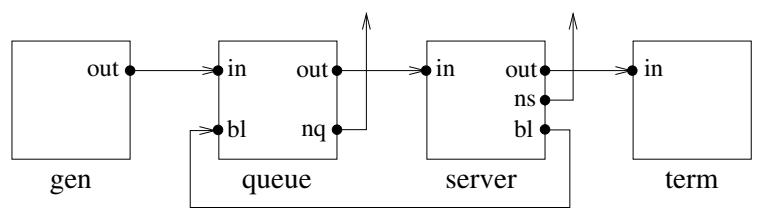

Figure 1: Example model singleserver.

Though this is probably by far the most studied system in discrete modeling, it is not trivial at all, especially if you try to model it with PDEVS. In the fundamental book of Zeigler et al. [3] a queue-server combination is modelled as one atomic component. But trying to create separate atomic models for a queue and a server is much more challenging due to the complex interaction of the two components.

The server can be implemented easily using the state diagram shown in Figure 2: When an entity $E$ arrives, the server outputs the new blocking status and changes to the "busy" state. After the service time, it outputs the entity and the changed blocking status and returns to the "idle" state. In this and the following figures the annotation $(A) B / C$ on an arrow means: If condition $A$ is true and input is $B$, then the output is $C$ and the state changes. Any of the three parts may be missing.

The behaviour of the queue is much more complicated, it is modelled here using the state diagram in Figure 3. The four states are distinguished by the size of the queue ("empty", "queuing") and the blocking status at

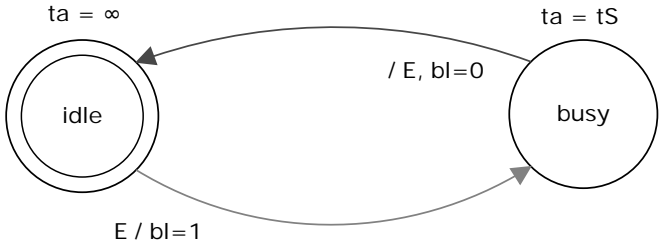

Figure 2: State diagram of the server component.

the output of the queue ("free", "blocked"). The only internal transitions occur in the state "queuing free", they output an entity and have zero transition time. All other transitions are external, triggered by an incoming entity or a new blocking status.

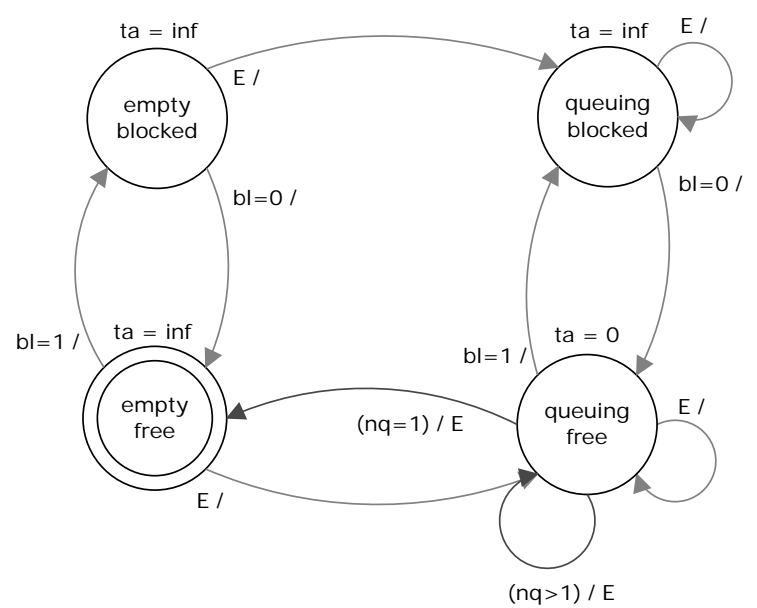

Figure 3: State diagram of the queue component.

This implementation of a queue uses a push strategy, where entities proceed as far as possible, until they are blocked. This is the basic idea behind many discrete event simulators, from GPSS to SimEvents. Alternatively, one could use a pull strategy, where entities only proceed, when they are called by a component. This would lead to a slightly simpler implementation of our example. It is an interesting question, whether pull or push strategies are better suited for complex simulation environments, but not the point of this investigation.

The simulation of the complete singleserver model leads to complicated cascades of concurrent events. For a typical example assume that the queue is in state "queuing blocked" with $n q>1$ and the server gets ready, going from "busy" to "idle". It sends its new blocking status $b l=0$ to the queue, which now transitions to "queuing free". Using an internal transition 
the queue outputs an entity, which arrives at the server, leading to a transition to "busy" and the sending of $b l=1$ back to the queue. Now the queue has to change to "queuing blocked", before another entity is output via an internal transition.

\section{Implementing Singleserver with PDEVS}

The fundamental component in the PDEVS formalism is an atomic PDEVS [3]. It is formally described by an 8-tuple $<X^{b}, S, Y^{b}$, ta $, \delta_{\text {int }}, \delta_{\text {ext }}, \delta_{\text {con }}, \lambda>$ with the meanings

$\begin{array}{ll}X^{b} & \text { set of possible input bags } \\ S & \text { set of states } \\ Y^{b} & \text { set of possible output bags } \\ \operatorname{ta}: S \rightarrow[0, \infty] & \text { time advance function } \\ \delta_{\text {int }}: S \rightarrow S & \text { internal transition function } \\ \delta_{\text {ext }}: Q \times X^{b} \rightarrow S & \text { external transition function } \\ \delta_{\text {con }}: S \times X^{b} \rightarrow S & \text { confluent transition function } \\ \lambda: S \rightarrow Y^{b} & \text { output function }\end{array}$

where an element of $\mathrm{Q}$ combines a state and the time since the last internal transition, i.e.

$$
Q:=\{(s, e) \mid s \in S, e \in[0, \operatorname{ta}(s)]\}
$$

It is important to note, especially for the present discussion, that the output function $\lambda$ is only called directly before an imminant internal transition.

Atomic components can be combined to form a $\mathrm{cou}$ pled PDEVS, which is formally defined by the set of components and their internal and outwards connections.

To implement the singleserver example in PDEVS one has to augment the state diagrams with transitional states that allow to produce output values, when an input appears, i. e. at an external event. For the server component (Fig. 2) one additional state is sufficient, and the definition of the transition, output and time advance functions is straighforward.

The definition of the queue component (Fig. 3) is much more complicated, its extended state diagram contains five additional states and a lot of corresponding additional transitions (Fig. 4). The purpose of the four states " $\mathrm{n}$ out $\mathrm{A} / \mathrm{B} / \mathrm{C} / \mathrm{D}$ " and their corresponding transitions is evident: Whenever an entity arrives, the length of the queue changes, and a corresponding output value has to be sent.

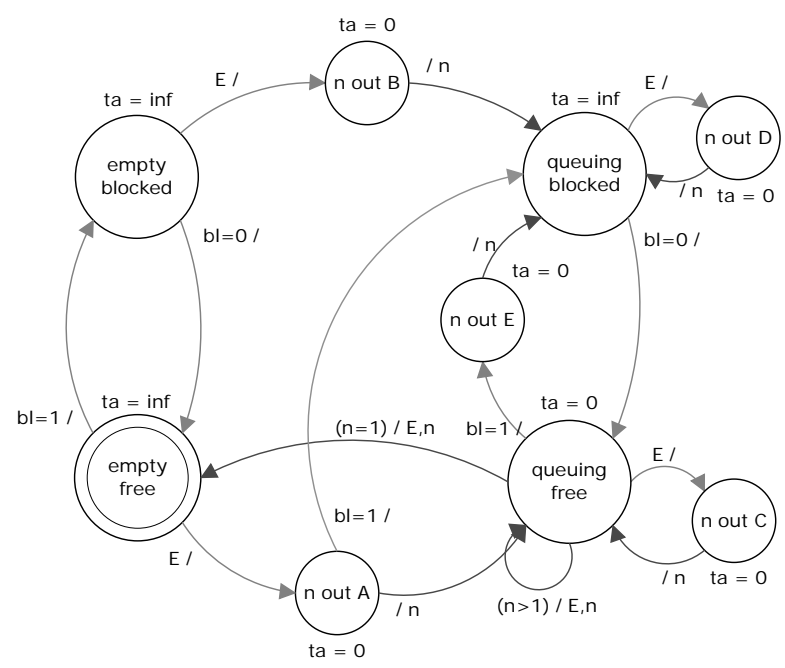

Figure 4: State diagram of the queue component with additional transient states.

But the new states lead to further complications, in particular for the definition of the external transition function $\delta_{\text {ext }}$ : When a new entity and a new blocking status arrive at the same time, the state has to proceed two "steps" at once to reach a necessary transitional state. E. g. when the queue is in state "queueing blocked" and a new entity arrives together with $b l=0$, the new entity is stored and the new state is "n out C".

Special care is needed for the definition of the confluent transition function $\delta_{\text {con }}$. Usually it first calls the internal, then the external transition function, so that the entity at the head of the queue leaves, before the new entity is stored. But if a new value $b l=1$ arrives, only the external transition function is used, so that no entity leaves the queue. This leads to a change of the queue size without a call of the internal transition function, therefore one needs another transitional state "n out E" to produce the corresponding output.

If one has taken proper care of all complications the complete model can be implemented in a PDEVS simulation environment like MatlabDEVS [13], where a simuation run will produce the expected results shown in Fig. 5. The "spikes" in the plots of the queue length and the server allocation are remnants of the concurrent event chains, where state variables have different values at the same time instant. 


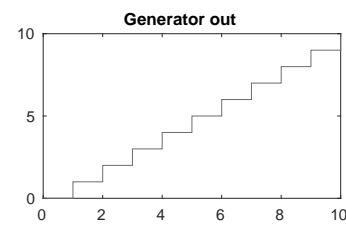

Queue $n$

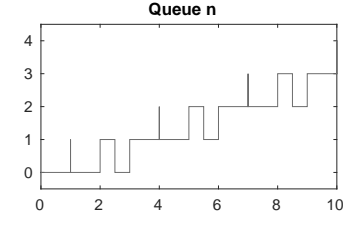

Server $\mathbf{n}$
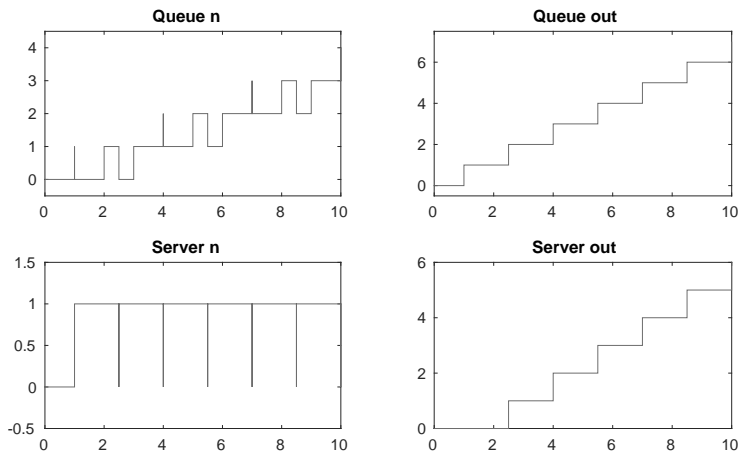

Server out

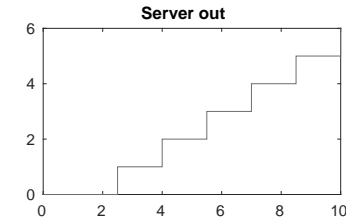

Figure 5: Simulation results of the PDEVS model.

\section{Trying to Implement Singleserver with RPDEVS}

To make the direct definition of Mealy components possible, Preyser et al. define RPDEVS in [10] as follows: An atomic RPDEVS is a simplified version of the atomic PDEVS, which contains only a generic transition $\delta$. Moreover its output funtion $\lambda$ is called at any kind of event and depends on the state and the input. Formally an atomic RPDEVS is given by a 6-tuple $<X^{b}, S, Y^{b}, t a, \delta, \lambda>$ with the meanings

$\begin{array}{ll}X^{b} & \text { set of possible input bags } \\ S & \text { set of states } \\ Y^{b} & \text { set of possible output bags } \\ t a: S \rightarrow[0, \infty] & \text { time advance function } \\ \delta: Q \times X^{b} \rightarrow S & \text { state transition function } \\ \lambda: Q \times X^{b} \rightarrow Y^{b} & \text { output function }\end{array}$

and the $\mathrm{Q}$ defined above.

A coupled RPDEVS is formally defined as in PDEVS, describing the subcomponents and the internal and outward connections. But its behaviour is different, in order to cope with possible Mealy components: The call of a $\lambda$-function produces outputs that are routed to other components, where they in turn may lead to a call of their $\lambda$-functions, creating cascades of $\lambda$ steps, which might change already processed input values. In such a case earlier input values are withdrawn and replaced by new ones (or cancelled completely).
For models without algebraic loops these $\lambda$ iterations will finally lead to a situation, where all input bags are constant. Only then a single $\delta$ call is issued.

The singleserver example can be formulated in RPDEVS much easier than in PDEVS, since one can stick to the simple state diagrams shown in Fig. 2 and Fig. 3. This allows to specify the atomic components in a straightforward way by identifying the event type inside the generic $\delta$ function according to the input bag and the elapsed time. Using the PowerRPDEVS simulator [14], the components can be easily implemented in $\mathrm{C}++$. Finally one can construct the complete singleserver example in a graphical environment.

Though all components work in simple test models, the simulation of the singleserver model aborts at $t=1$. The error message states that the maximum number of $\lambda$ steps has been reached and that the model is illegitimate due to a non-resolvable algebraic loop.

The reason for this behaviour becomes clear, when one analyses the internal chain of events in the simulator at $\mathrm{t}=1$ (cf. Table 1): The queue starts in state "empty free" and changes to state "queuing free" in line 3 , while the server remains in state "idle". In line 4 the queue outputs its entity that is routed to the server, which now sends the blocking status $\mathrm{bl}=1$ to the queue. The basic problem now happens in line 6 : The $\lambda$ function of the queue is called again, now with $\mathrm{bl}=1$ in the input bag. The entity that has been sent before, is now blocked and has to be retrieved. This in turn leads to the withdrawal of the $b l=1$ message from the server, therefore the queue tries again to output its entity in line 7. The situation is now identical to line 4 and repeats, until it is stopped, when the maximal count of $\lambda$ steps is reached.

\begin{tabular}{c|ccc|ccc} 
No. & Block & Type & Out & Q in & Q bl & S in \\
\hline 1 & Gen & $\lambda$ & E1 & E1 & & \\
2 & Que & $\lambda$ & & & & \\
3 & Que & $\delta$ & & & & \\
4 & Que & $\lambda$ & E1 & & & E1 \\
5 & Srv & $\lambda$ & bl=1 & & 1 & \\
6 & Que & $\lambda$ & & & & \\
7 & Que & $\lambda$ & E1 & & & E1
\end{tabular}

Table 1: Events at $\mathrm{t}=1$.

The basic idea behind the state diagram in Fig. 3 was, that a component changes its state immediately after sending its output message. Therefore new input messages, that arrive due to event cascades, find the 
component in a changed state. But the repeated execution of $\lambda$ steps without any state changing $\delta$ steps in RPDEVS leads to a completely different behaviour.

Of course, this doesn't mean that it is impossible to implement working queue and server components with RPDEVS: One could mimic the PDEVS behaviour and add additional transitional states or one could change the internal behaviour altogether and use a different scheme based on another state diagram. But a simple direct implementation of Fig. 3 doesn't work.

From the point of view of RPDEVS the singleserver is faulty, containing an algebraic loop. On the other hand the behaviour described in Fig. 3 is quite simple and can be easily implemented in PDEVS. Zeigler knew very well, why he didn't include Mealy-type behaviour. But if one insists on it for the sake of better modularity, one has to think over the simulator behaviour, or better: the abstract model behind it.

\section{Extending the Time Line by Infinitesimals}

Modeling experience teaches us that a mathematical problem in the description or simulation of a model often has its roots in an oversimplification of the system one wants to describe. This is of course true here: In real world systems small delay times are inevitable, whenever a message is sent or a state changes. If one includes them in the model description, the problems with cascades of concurrent events disappear immediately. But the price one has to pay for this solution, is high: Such a model contains a huge amount of delay times, whose values are not known, often not even their order of magnitude. In addition, the behaviour of the model gets much more complicated on a fine time scale, though one often is not interested in these details.

What we are looking for, are time steps that are larger than zero, but so small that they can be ignored for most purposes. Furthermore their actual size should not matter, even though we need different sizes of such steps. This is actually exactly what one commonly denotes as infinitesimals. Hewitt [15] and Robinson [16] have shown that one can implement such ideas in a mathematically rigorous manner. Therefore we will shortly introduce the basic concepts and use them afterwards for a new definition of discrete event systems. A precise and pedagogical introduction to the mathematical ideas and applications can be found in [12].

The set ${ }^{*} \mathbb{R}$ of hyperreals is a totally ordered field that includes the ordinary real numbers. In addition it contains an infinitesimal element $\varepsilon>0$ that is smaller than any positive real number. Using the field axioms one gets additional infinitesimals like $2 \varepsilon,-\varepsilon, \varepsilon^{2}$. Each real number $r$ is surrounded by an infinite cloud $r+\delta$ with infinitesimal $\delta$, its halo. On the other end ${ }^{*} \mathbb{R}$ contains $\omega:=1 / \varepsilon$, which is unlimited, i. e. larger than any real number. Again one has lots of unlimited numbers like $2 \omega,-\omega, \omega^{2}$, which are all surrounded by clouds $\omega+r+\delta$ with real $r$ and infinitesimal $\delta$, called their galaxy. Each limited element $h \in{ }^{*} \mathbb{R}$, i. e. an element of the galaxy of 0 , can be uniquely written as $h=r+\delta$ with real $r$ and infinitesimal $\delta . r$ is called the standard part st $(h)$ of $h$.

The actual construction of $* \mathbb{R}$ relies on heavy machinery from set theory and logic, like ultrafilters and the axiom of choice. From our current point of view the main reason for an explicit construction is to convince oneself that such a set exists in a precise mathematical way. Hyperreals have been used to reformulate the usual analysis with definitions that closely mimic the original ideas of Leibniz, an endeavor commonly designated as nonstandard analysis. This often leads to simple and intuitive proofs - once one accepts the basic properties of $* \mathbb{R}$.

It is impossible to implement real numbers in a computer, much less hyperreals. For our purposes it is sufficient to use pairs $(t, r)$ of floating point numbers, which correspond to the hyperreal $t+r \varepsilon$, where $t$ could be the floating point value $\infty$ to include infinite time delays in passive states. This implementation looks similar to the concept of superdense time [17] that uses a pair of a real time value and a natural number for ordering of concurrent events. But the structure of the hyperreals is much richer, and the reasoning behind their use is more intuitive and better adapted to the problems that are adressed here.

\section{Definition of NSA-DEVS}

We will now use non-standard analysis ("NSA") to get rid of concurrent events by defining NSA-DEVS, a variant of RPDEVS. The basic idea is to forbid transient states, i. e. transition times are always $>0$, though they may be infinitesimal. Furthermore we assume that the transport of data between components always takes a certain amount of time. Therefore we include an input delay $\tau>0$ between the arrival of input and the call of the output function. 
An atomic NSA-DEVS is given by a 7-tuple $<X^{b}, S, Y^{b}, \tau, t a, \delta, \lambda>$, where $\tau \in{ }^{*} \mathbb{R}_{>0}$ is the input delay time. All other elements have the same meaning as in RPDEVS, but the definitions of the functions are changed to

$$
\begin{aligned}
& Q:=\{(s, e) \mid s \in S, e \in(0, \operatorname{ta}(s)]\} \\
& \operatorname{ta}: S \rightarrow(0, \omega] \\
& \delta: Q \times X^{b} \rightarrow S \\
& \lambda: Q \times X^{b} \rightarrow Y^{b}
\end{aligned}
$$

The intervals $(0, t a(s)]$ and $(0, \omega]$ are meant as subsets of the hyperreals $* \mathbb{R}$.

When an external event, i.e. an input $x \in X^{b}$, occurs at time $t$, the output function $\lambda$ is called at $t+\tau$, followed by an immediate call of $\delta$. An internal event, i.e. an imminent state change after a waiting time $t a(s)$, leads to a direct (undelayed) call of $\lambda$ and $\delta$. A concurrent incidence of a (delayed) external event and an internal event can be detected by both functions directly and doesn't need a special mechanism.

A coupled NSA-DEVS is defined as in RPDEVS and PDEVS, outputs are transported as usual. Due to the (infinitesimal) delays a strict Mealy-type behaviour is impossible, therefore special provisions like the iterated $\lambda$ calls in RPDEVS are unnecessary, each $\lambda$ call is followed immediately by the corresponding $\delta$ call.

Formally we have introduced a lot of additional (infinitesimal) parameters, but a simulator might be able to free the user from this burden by using a simple default value of $\tau=\varepsilon$ for all components. Furthermore the transition times of previously transient states could be set to $\varepsilon$ in many cases. It remains to be seen, whether such a simple scheme actually works in standard situations. In the case of the singleserver example manual finetuning is necessary, as will be shown below. On the other hand, if one insists on a special ordering of (ideally) concurrent events, one can use the infinitesimal delays to achieve any order in a quite intuitive way.

Since one is generally not interested in the infinitesimal behaviour, an NSA-DEVS simulator should show state changes and output values only at the end of an infinitesimal cascade, i.e. directly before a finite (noninfinitesimal) step. All short-lived states and overwritten outputs are then internal to the simulator. This behaviour is somewhat similar to that of an ODE solver that uses microsteps internally for stepsize adaptation, but outputs only completed steps. Optionally it should be possible to replace the value $\varepsilon$ by a user supplied small real number for debugging purposes or to analyse the behaviour at the infinitesimal scale.

\section{Implementing Singleserver with NSA-DEVS}

To adapt the RPDEVS description of singleserver to NSA-DEVS, one needs two modifications: All components get an additional parameter $\tau$ with a default value of $\varepsilon$, and the queue component gets a further parameter $t_{D}$, which defines the transition time of the state "queuing free", again with a default value of $\varepsilon$.

Until a proper NSA-DEVS simulator is available, one can use the PowerRPDEVS simulator to mimic the behaviour in debug mode, where the infinitesimal value $\varepsilon$ is set to a small number $\left(\varepsilon=10^{-4}\right.$ in the following examples). To this end one creates a simple delay component, basically a simple server, and adds it before every input of each component. All delay times are set to the value of $\tau$ of the corresponding component. In particular, the delay times of several inputs of one component have to be identical to properly implement the NSADEVS behaviour defined above. These delays guarantee that no $\lambda$ iterations occur, therefore the simulator works as required by the NSA-DEVS definition.
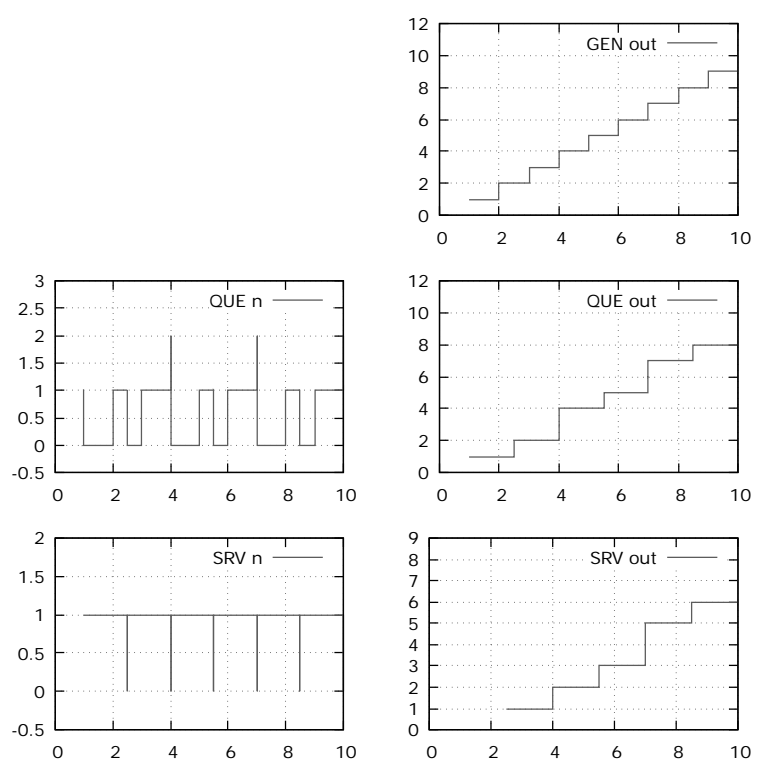

Figure 6: Simulation results of the NSA-DEVS model with default delays.

With these changes the singleserver model runs in "NSA-DEVS debug mode", the results are shown in 
Fig. 6. But they are not as expected: At $t=4$ the entity 4 joins entity 3 in the queue and both leave the queue immediately. After the service time entity 3 leaves the server at $t=5.5$, but entity 4 is lost. At first thought this problem seems to be related to the nullserver problem described in [18]: The delay at the input of the server acts as additional storage and accepts entity 4 though the server is busy.

While this is true, the real cause of the problem lies elsewhere. Even in a correct implementation of NSADEVS, where the delay is implemented directly in the simulator, entity 4 would get lost! This is due to the delay of the "busy" message from the server: Before it arrives, the queue has already output the next entity according to the internal transition shown in Fig. 3. This is an example, where it is not sufficient to use the default value $\varepsilon$ for all infinitesimal delays.

In order to obtain the desired causal ordering of "concurrent" events, a fine tuning of the delays is necessary. One has to guarantee that the message from the server arrives, before the queue sends a new entity. Therefore one sets the transition time $t_{D}$ of the "queuing free" state to a value larger than the sum of the two transport delays at the inputs of the server and the queue. Choosing $t_{d}=2.1 \varepsilon$ solves the problem and the results are as expected. They coincide with the results of the PDEVS implementation (cf. Fig. 5), including the "spikes". Here they have a small, but finite width, like it should be in a proper NSA-DEVS simulator in debug mode. In standard mode output values that change in an infinitesimal time are suppressed and only the last values are shown.

\section{Conclusions}

Like RPDEVS, the variant NSA-DEVS proposed here allows for simple and consistent handling of Mealytype behaviour. Furthermore it seems to solve the problems of RPDEVS with chains of concurrent events. Therefore it possibly could be a working basis for a concrete simulation environment.

The drastic measure of prohibiting real "concurrency" for causally ordered events generally causes serious side effects by introducing lots of additional time parameters. This is mitigated here by the introduction of infinitesimal delays used mainly internally and whose actual values do not matter. That such a scheme is mathematically sound has been shown by referring to the results of non-standard analysis.
A certain amount of fine-tuning can still be necessary to ensure a requested causal ordering. But this is not a speciality of NSA-DEVS: The proper behaviour of the system has to be modelled anyhow, in PDEVS this is done by a careful design of the confluent transition function. In case of causally unrelated events, one could twist some of the infinitesimal delay parameters to ensure a certain temporal order, if requested.

At first sight NSA-DEVS seems to destroy the potential of parallel execution. But this is not necessarily the case: For unrelated events one simply chooses identical delays - usually just $\varepsilon$-, so that they still occur at the same time $t \in{ }^{*} \mathbb{R}$ and can be executed in parallel. Only causally depending events have different times, so that their order is fixed - as it should be. Moreover, the elimination of many transitional states in RPDEVS and NSA-DEVS could provide more opportunities for parallel execution than PDEVS.

The difference between RPDEVS and NSA-DEVS is due to the fact that they address different problems: RPDEVS copes with concurrent events that are causally unrelated, as they appear in models using Mealy-type components. In contrast NSA-DEVS deals primarily with concurrent events that are causally related, i. e. have a logical order. While RPDEVS offers a completely internal solution for the first problem, similar to the internal event loop in Modelica [19], NSA-DEVS reveals internal details to the modeller, which can be helpful in some cases, but might be confusing in others. Whether this approach is feasible in complex models, has to be seen in future studies.

This is only a first step in the analysis of a new DEVS-based scheme that could possibly be used for simulation environments and the definition of universally applicable component libraries. The next step would be the definition and implementation of a proper simulator. This could be followed by a thorough investigation of standard examples with complex event cascades, such as a switch that routes entities according to an input value [9] or models of digital circuits containing flip-flops [20, 21]. Finally one could try to implement a complex case study like the ARGESIM C22 benchmark [22] as a further step to investigate the practical usefulness of the proposed scheme.

\section{Acknowledgement}

The author gratefully acknowledges clarifying discussions with Christina Deatcu, Thorsten Pawletta and Franz Preyser. 


\section{References}

[1] Zeigler BP. Theory of Modeling and Simulation. New York: Wiley-Interscience, 1st ed. 1976.

[2] Chow ACH. Parallel DEVS: A Parallel, Hierarchical, Modular Modeling Formalism and its Distributed Simulators. Transactions of The Society for Computer Simulation International. 1996;13(2):55-67.

[3] Zeigler BP, Praehofer H, Kim TG. Theory of Modeling and Simulation. San Diego: Academic Press, 2nd ed. 2000.

[4] W David Kelton NBZ Randall P Sadowski. Simulation with Arena. New York: McGraw-Hill, 6th ed. 2015.

[5] Clune MI, Mosterman PJ, Cassandras CG. Discrete Event and Hybrid System Simulation with SimEvents. In: 8th International Workshop on Discrete Event Systems. Ann Arbor. 2006; pp. 386-387.

[6] Li W, Mani R, Mosterman PJ. Extensible discrete-event simulation framework in SimEvents. In: Proc. 2016 Winter Simulation Conference. Arlington: IEEE. 2016; pp. 943-954.

[7] Franceschini R, Bisgambiglia PA, Touraille L, Bisgambiglia P, Hill D. A survey of modelling and simulation software frameworks using Discrete Event System Specification. In: Proc. of 2014 Imperial College Computing Student Workshop. Schloss Dagstuhl-Leibniz-Zentrum fuer Informatik. 2014; pp. 40-49.

[8] Goldstein R, Breslav S, Khan A. Informal DEVS conventions motivated by practical considerations. In: Proc. of Symposium on Theory of Modeling \& Simulation - DEVS Integrative M\&S Symposium. 2013; pp. 10:1-10:6.

[9] Preyser FJ, Heinzl B, Raich P, Kastner W. Towards Extending the Parallel-DEVS Formalism to Improve Component Modularity. In: Proc. of ASIM-Workshop STS/GMMS. Lippstadt. 2016; pp. 83-89.

[10] Preyser FJ, Heinzl B, Kastner W. RPDEVS: Revising the Parallel Discrete Event System Specification. In: 9 th Vienna Int. Conf. Mathematical Modelling. Wien. 2018; pp. 242-247.

[11] Preyser FJ, Heinzl B, Kastner W. RPDEVS Abstract Simulator. SNE Simulation Notes Europe. 2019; 29(2):79-84. doi: 10.11128/sne.29.tn.10473.

[12] Goldblatt R. Lectures on the Hyperreals. New York: Springer. 1998.

[13] Pawletta T, Deatcu C, Pawletta S, Hagendorf O, Colquhoun G. DEVS-based modeling and simulation in scientific and technical computing environments. In:
Proc. of DEVS Integrative M\&S Symposium (DEVS'06)

- Part of the 2006 Spring Simulation Multiconference (SpringSim'06). Huntsville/AL, USA: D. Hamilton. 2006; pp. 151-158.

[14] Preyser F. PowerRPDEVS on sourceforge. URL https: / / sourceforge. net/projects / powerrpdevs /

[15] Hewitt E. Rings of real-valued continuous functions I. Transactions of the American Mathematical Society. 1948;64(1):45-99.

[16] Robinson A. Non-standard Analysis. Amsterdam: North-Holland. 1966.

[17] Sarjoughian HS, Sundaramoorthi S. Superdense time trajectories for DEVS simulation models. In: SpringSim (TMS-DEVS). 2015; pp. 249-256.

[18] Austermann L, Junglas P, Schmidt J, Tiekmann C. Conceptional problems of transaction-based modeling and its implementation in SimEvents 4.4. SNE Simulation Notes Europe. 2017;27(3):137-142. doi: 10.11128/sne.27.tn.10383.

[19] Modelica Association. Modelica $\AA$ - A Unified Object-Oriented Language for Systems Modeling, Language Specification Version 3.5.

URL https :

//modelica.org/documents/MLS.pdf

[20] Fiedler C, Preyser FJ, Kastner W. Simulation of RPDEVS Models of Logic Gates. SNE Simulation Notes Europe. 2019;29(2):85-91. doi: 10.11128/sne.29.tn.10474.

[21] Junglas P. Pitfalls using discrete event blocks in Simulink and Modelica. In: Proc. of ASIM-Workshop STS/GMMS. Lippstadt. 2016; pp. 90-97.

[22] Junglas P, Pawletta T. Non-standard Queuing Policies: Definition of ARGESIM Benchmark C22. SNE Simulation Notes Europe. 2019;29(3):111-115. doi: 10.11128/sne.29.bn22.10481. 


\title{
SyNumSeS: A Python Package for Numerical Simulation of Semiconductor Devices
}

\author{
Peter Abele ${ }^{1 *}$, Michael Schäfer $^{2}$ \\ ${ }^{1}$ HTWG Konstanz - University of Applied Sciences, 78462 Konstanz, Alfred-Wachtel-Str. 8, Germany \\ *pabele@htwg-konstanz.de \\ ${ }^{2}$ CADwalk GmbH \& Co. KG, Joseph-von-Sontheimer-Str. 3, 89604 Allmendingen, Germany
}

SNE 31(2), 2021, 81-85, DOI: 10.11128/sne.31.sw.10566 Received: May 26, 2021 (Selected ASIM WS GMMS/STS 2021 Postconf. Publ.), Accepted: June 5, 2021

SNE - Simulation Notes Europe, ARGESIM Publisher Vienna, ISSN Print 2305-9974, Online 2306-0271, www.sne-journal.org

Abstract. SyNumSeS is a Python package for numerical simulation of semiconductor devices. It uses the Scharfetter-Gummel discretization for solving the one dimensional Van Roosbroeck system which describes the free electron and hole transport by the drift-diffusion model. As boundary conditions voltages can be applied to Ohmic contacts. It is suited for the simulation of pndiodes, MOS-diodes, LEDs (hetero junction), solar cells, and (hetero) bipolar transistors.

\section{Introduction}

The development of modern semiconductor devices is accompanied by numerical simulations. This results in a better understanding of the device as physical quantities like electrical fields or current densities can be visualized and related to material parameters or the geometry of the device. This way time consuming experiments can be reduced saving resources, time, and money. In teaching semiconductor devices the visualization of what is happening inside the device is very helpful for the students. So they can get a better link between the learned equations and what do they mean for the electrical properties of the device. Presenting animations showing the output characteristics and for example the hole and electron density in the device for the different bias regions is often better then thousands of words. This was the motivation for programming a simple to use and fast Python package for numerical simulations of semiconductor devices.

This package is used for the lecture 'Electron Devices' to generate diagrams and animations. In the mas- ter's course 'Microfabrication Techniques' the students use the package within a lab for the simulations of pndiodes, LEDs, solar-cells and SiGe hetero bipolar transistors.

\section{Solving the Basic Semiconductor Equations}

The Van Roosbroeck equations [1] are used as the basic semiconductor equations. For one dimension they are:

$$
\begin{array}{r}
-\frac{\partial}{\partial x}\left(\varepsilon_{0} \varepsilon_{r} \frac{\partial}{\partial x} \Psi\right)=q_{\mathrm{e}} \cdot(C+p-n), \\
\frac{\partial}{\partial t} n=\frac{1}{q_{\mathrm{e}}} \cdot \frac{\partial}{\partial x} j_{\mathrm{n}}+(G-R), \\
\frac{\partial}{\partial t} p=-\frac{1}{q_{\mathrm{e}}} \cdot \frac{\partial}{\partial x} j_{\mathrm{p}}+(G-R) .
\end{array}
$$

These are three nonlinear ordinary differential equations for three unknowns, the potential $\Psi$, the free electron density $n$, and the free hole density $p$. ( $C$ is the doping concentration, $G$ and $R$ are the generation and recombination rates, respectively, $\varepsilon_{0}$ is the dielectric constant, and $\varepsilon_{r}$ is the relative permittivity.) These equations can be solved by using partial differential equation (PDE) solvers like FiPy[2]. As for semiconductor devices high electrical fields and extreme concentration gradients for electrons and holes exist especially at pnor hetero-junctions using a general PDE solver results in significant deviation from the exact solution. This can be partly compensated by reducing the grid size but at the expense of simulation time and even convergence issues.

By using the Scharfetter Gummel scheme [3][4] for the electron and hole current densities this issue can be relaxed. 
Additionally in the equations (1) the unknowns $n$ and $p$ are replaced by the quasi Fermi levels for electrons $\Phi_{\mathrm{n}}$ and holes $\Phi_{\mathrm{p}}$, respectively using the flowing relations:

$$
n=N_{\mathrm{C}} \cdot \exp \left(\frac{X-\Phi_{\mathrm{n}}+\Psi}{U_{\mathrm{t}}}\right)
$$

and

$$
p=N_{\mathrm{V}} \cdot \exp \left(\frac{-X-E_{\mathrm{g}}+\Phi_{\mathrm{p}}-\Psi}{U_{\mathrm{t}}}\right)
$$

With $X^{1}$ being the electron affinity, $E_{\mathrm{g}}$ the band gap, and $U_{\mathrm{t}}=\frac{k_{\mathrm{B}} T}{q_{\mathrm{e}}}\left(k_{\mathrm{B}}\right.$ Boltzmann constant, $T$ absolute temperature, and $q_{\mathrm{e}}$ elementary charge). $N_{\mathrm{C}}$ and $N_{\mathrm{V}}$ are the effective density of states for the conduction band and the valence band, respectively.

For solving this PDEs the finite volume method for one dimension is used. The electron and hole current densities between two adjacent cells are expressed by:

$$
\begin{aligned}
& j_{\mathrm{n} ; \mathrm{k}, \mathrm{l}}= \\
& -\frac{q_{\mathrm{e}} U_{\mathrm{t}}}{\Delta x} \cdot \frac{\mu_{\mathrm{n}, \mathrm{k}}+\mu_{\mathrm{n}, \mathrm{l}}}{2} \cdot \frac{N_{\mathrm{C}, \mathrm{k}}+N_{\mathrm{C}, \mathrm{l}}}{2} \cdot \exp \left(\frac{X_{\mathrm{k}}+X_{1}}{2}\right) \cdot[ \\
& B\left(-\frac{\Psi_{1}-\Psi_{\mathrm{k}}}{U_{\mathrm{t}}}\right) \cdot \exp \left(\frac{\Psi_{\mathrm{k}}-\Phi_{\mathrm{n}, \mathrm{k}}}{U_{\mathrm{t}}}\right) \\
& -B\left(\frac{\Psi_{1}-\Psi_{\mathrm{k}}}{U_{\mathrm{t}}}\right) \cdot \exp \left(\frac{\Psi_{1}-\Phi_{\mathrm{n}, 1}}{U_{\mathrm{t}}}\right) \\
& j_{\mathrm{p} ; \mathrm{k}, \mathrm{l}}= \\
& -\frac{q_{\mathrm{e}} U_{\mathrm{t}}}{\Delta x} \cdot \frac{\mu_{\mathrm{p}, \mathrm{k}}+\mu_{\mathrm{p}, \mathrm{l}}}{2} \cdot \frac{N_{\mathrm{V}, \mathrm{k}}+N_{\mathrm{V}, 1}}{2} \cdot \exp \left(\frac{X_{\mathrm{k}}+E_{\mathrm{g}, \mathrm{k}}+X_{\mathrm{l}}+E_{\mathrm{g}, 1}}{2}\right) \cdot[ \\
& B\left(-\frac{\Psi_{1}-\Psi_{\mathrm{k}}}{U_{\mathrm{t}}}\right) \cdot \exp \left(\frac{\Phi_{\mathrm{p}, \mathrm{k}}-\Psi_{\mathrm{k}}}{U_{\mathrm{t}}}\right) \\
& -B\left(\frac{\Psi_{1}-\Psi_{\mathrm{k}}}{U_{\mathrm{t}}}\right) \cdot \exp \left(\frac{\Phi_{\mathrm{p}, 1}-\Psi_{1}}{U_{\mathrm{t}}}\right)
\end{aligned}
$$

With $k$ and $l$ being indices of adjacent cells, $\mu_{\mathrm{n}}$ and $\mu_{\mathrm{p}}$ are the mobilities of electrons and holes, respectively, and $\Delta x$ is the cell distance. $B(x)$ is the Bernoulli function:

$$
B(x)=\frac{x}{\exp (x)-1} .
$$

Compared to [4] the equations (4) and (5) are slightly modified by the terms containing the electron affinity $X$ to account for hetero junctions.

Care must be taken for the Bernoulli function in the case of low values for the argument as this results in a low value divided by an other low value. Therefore the Bernoulli function is approximated by a polynomial of degree 1 for low values of the argument:

$$
B(x)=1-\frac{x}{2}
$$

\section{Boundary Condition}

On the very left and right of the one dimensional device boundary conditions for Ohmic contacts are set and different voltages can be applied to both sides. This is done by adding one additional cell on the left and right with the quasi Fermi levels and potential set to [5]:

$$
\begin{gathered}
\Phi_{\mathrm{n}}=V_{\mathrm{A}} \\
\Phi_{\mathrm{p}}=V_{\mathrm{A}} \\
\Psi=-X-\frac{E_{\mathrm{g}}+U_{\mathrm{t}} \log \left(\frac{N_{\mathrm{C}}}{N_{\mathrm{V}}}\right)}{2}+U_{\mathrm{t}} \operatorname{arcsinh}\left(\frac{C}{2 n_{\mathrm{i}}}\right)+V_{\mathrm{A}}
\end{gathered}
$$

With $V_{\mathrm{A}}$ the applied voltage and $n_{\mathrm{i}}$ the intrinsic carrier density. For the simulation of bipolar transistors an additional contact between the outer contacts is needed. For this contact dependent on the doping, p- or n-type, only the potential $\Psi$ and one of the quasi Fermi levels $\Phi_{\mathrm{p}}$ or $\Phi_{\mathrm{n}}$ are set, respectively.

\section{Solving the Semiconductor Equations}

The discretization of the equations (1) using the equations (2)-(5) results in a nonlinear equation system for the unknowns $\Psi, \Phi_{\mathrm{n}}$, and $\Phi_{\mathrm{p}}$ for every cell. To solve this nonlinear equation system Newton's method is used. For building the Jacobian matrix the equations are symbolically derived using the Python package sympy. The code for solving these equations is automatically generated by a Python script.

\footnotetext{
${ }^{1}$ For ease of use the units of $X, E_{\mathrm{g}}, \Phi_{\mathrm{n}}$, and $\Phi_{\mathrm{p}}$ are $\mathrm{V}$ and not $\mathrm{eV}$ !
} 


\section{Simulation Results}

To demonstrate the wide use of this package simulation results for three different semiconductor devices are presented.

\subsection{Simulation results of an InGaN LED}

For the simulation of an $\mathrm{LED}^{2}$ the InGaN material system is used. The well, where the light is generated, consists of $\operatorname{In}_{0.3} \mathrm{Ga}_{0.7} \mathrm{~N}$ with a band gap of about $E_{\mathrm{g}}=$ $2.0 \mathrm{eV}$ and has a thickness of $100 \mathrm{~nm}$. The surrounding material is $\mathrm{GaN}$ with $E_{\mathrm{g}}=3.4 \mathrm{eV}$. The $\mathrm{In}_{0.3} \mathrm{Ga}_{0.7} \mathrm{~N}$ well is not doped while the surrounding $\mathrm{GaN}$ to the left is $\mathrm{p}$-doped and to the right is n-doped both with a concentration of $N_{\mathrm{A}}=N_{\mathrm{D}}=2 \cdot 10^{22} \mathrm{~m}^{-3}$.

Fig. 1 shows the resulting band diagram with no externally applied voltage.

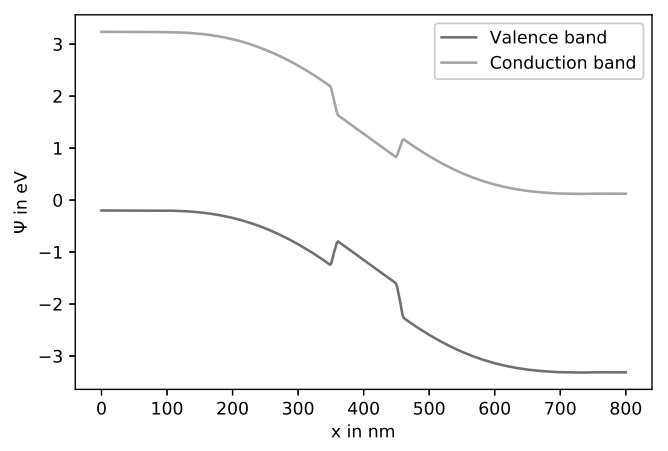

Figure 1: Band diagram of InGaN-LED with no externally applied voltage.

In the center the $\operatorname{In}_{0.3} \mathrm{Ga}_{0.7} \mathrm{~N}$ well can be clearly seen and the band bending respectively build in voltage $\Phi_{\mathrm{bi}}$ due to the doping concentrations. Simulating the output characteristics shows a diode threshold voltage of about $V_{\text {th }}=2.8 \mathrm{~V}$, see Fig. 2.

The nearly linear increase of the current density beyond $V_{\text {th }}$ can be attributed to the low doping level of the $\mathrm{GaN}$ resulting in a relatively high Ohmic resistance.

The recombination rate is considered by:

$$
R=C_{\mathrm{rad}} \cdot\left(n p-n_{\mathrm{i}}^{2}\right) .
$$

As the free electrons and holes accumulate at the well we get in this region the highest recombination rate, see Fig. 3.

\footnotetext{
${ }^{2}$ Light emitting diode
}

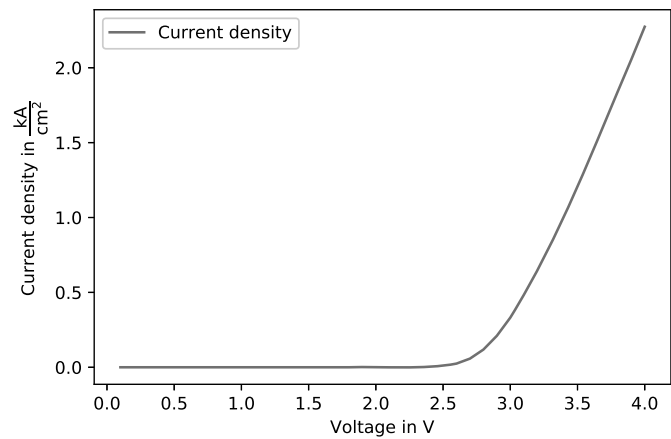

Figure 2: LED characteristic.

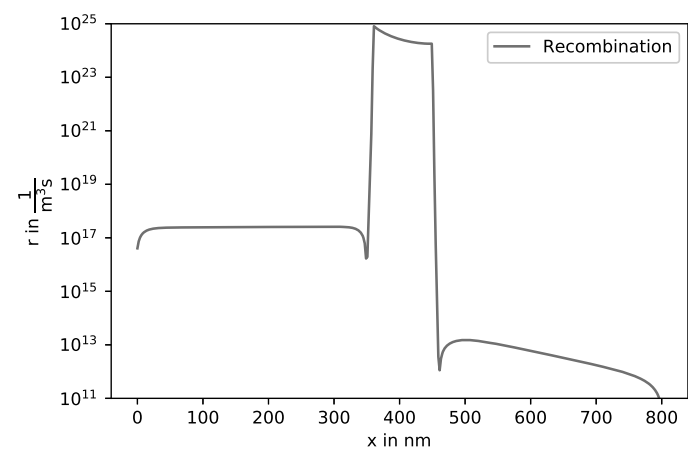

Figure 3: Recombination rate along the LED.

\subsection{Simulation results of solar cells}

In the following silicon solar cells are simulated. The thickness of the silicon is $d=1 \mu \mathrm{m}$. The first $40 \mathrm{~nm}$ from the surface are p-doped with $N_{\mathrm{A}}=1.0 \cdot 10^{24} \mathrm{~m}^{-3}$. The rest of the silicon is n-doped with different concentrations. The intensity of the incident light drops exponentially while penetrating through the silicon and generate free electrons and holes.

In the following the influence of the different ndoping concentrations, $N_{\mathrm{D}}=1.25 \cdot 10^{21} \mathrm{~m}^{-3}, N_{\mathrm{D}}=1.0$. $10^{22} \mathrm{~m}^{-3}$, and $N_{\mathrm{D}}=8.0 \cdot 10^{22} \mathrm{~m}^{-3}$ is shown. The intensity of the incident light is kept constant.

The doping level has two effects, first on the build in voltage $\Phi_{\mathrm{bi}}$, which increases with increasing doping level, and second the extension of the space charge region which decreases with increasing doping level. A higher build in voltage results in a higher idle voltage, this can be seen in Fig. 4. But on the other side with a reduction of the extension of the space charge region the short circuit current drops. 


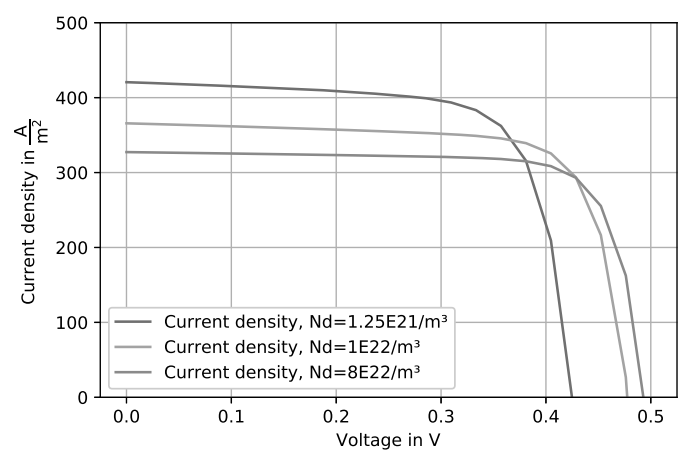

Figure 4: Solar cell output characteristic for different n-doping concentrations.

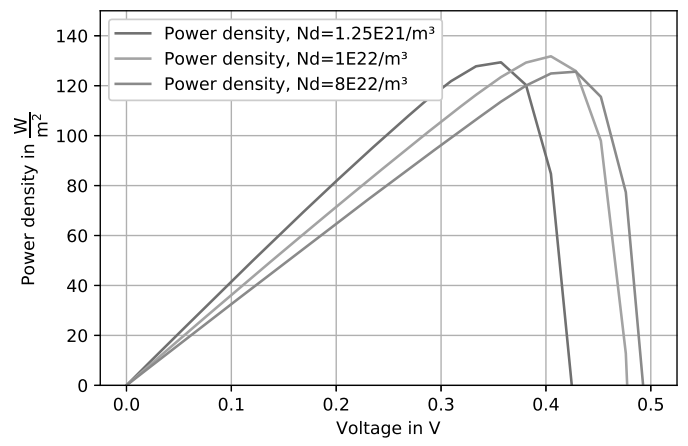

Figure 5: Output power density of differently doped solar cells.

For optimizing the doping concentration in Fig. 5 the power density dependent on the applied voltage is shown. The maximum power point is for the doping levels of $N_{\mathrm{D}}=1.25 \cdot 10^{21} \mathrm{~m}^{-3}$ and $N_{\mathrm{D}}=1.0 \cdot 10^{22} \mathrm{~m}^{-3}$ about the same. For the higher doping level it is about $5 \%$ lower.

\subsection{Simulation results of a Si bipolar transistor}

The following shows simulation results of a npn bipolar transistor. The device has an extension of $1.2 \mu \mathrm{m}$. The first $400 \mathrm{~nm}$ build the emitter which is n-doped with $N_{\mathrm{D}, \mathrm{E}}=1.2 \cdot 10^{24} \mathrm{~m}^{-3}$. The next $400 \mathrm{~nm}$ build the base which is p-doped with $N_{\mathrm{A}, \mathrm{B}}=4.0 \cdot 10^{22} \mathrm{~m}^{-3}$. The last $400 \mathrm{~nm}$ build the collector which is n-doped with $N_{\mathrm{D}, \mathrm{C}}=2.0 \cdot 10^{22} \mathrm{~m}^{-3}$.
For this simulation the base contact is located at $x=$ $650 \mathrm{~nm}$. As SyNumSeS can only solve devices for one dimension, the boundary condition at the base contact (p-doped) only fixes the potential $\Psi$ and the quasi Fermi level for the holes $\Phi_{\mathrm{p}}$. The quasi Fermi level for the electrons $\Phi_{\mathrm{n}}$ at this point is calculated by the nonlinear equation system. Thereby the electrons can pass the base contact.

In a first step the base emitter voltage is ramped from $V_{\mathrm{BE}}=0 \mathrm{~V}$ to $V_{\mathrm{BE}}=0.6 \mathrm{~V}$ in steps of $0.1 \mathrm{~V}$. Then the collector emitter voltage is ramped to $V_{\mathrm{CE}}=3.0 \mathrm{~V}$ in steps of $50 \mathrm{mV}$. This results in the output characteristics and is show in Fig. 6.

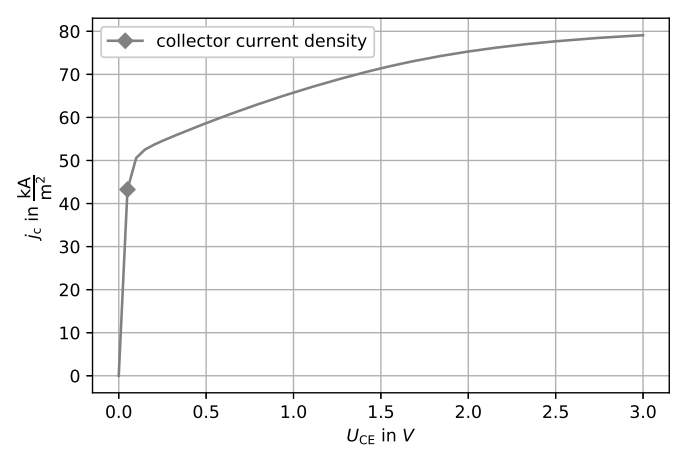

Figure 6: Output characteristics of a Si bipolar transistor for

$$
V_{\mathrm{BE}}=0.6 \mathrm{~V} \text {. }
$$

The simulated output characteristics shows the saturation region up to about $V_{\mathrm{CE}}=100 \mathrm{mV}$. The following slope can be attributed to the Early effect.

To get a better understanding for the saturation region, we can have a look at the electron and hole current density across the device at $V_{\mathrm{CE}}=50 \mathrm{mV}$ and $V_{\mathrm{BE}}=0.6 \mathrm{~V}$, see Fig. 7 .

It can be clearly seen that electrons are not only injected from the emitter into the base, but also from the collector into the base $\left(400 \mathrm{~nm}<x_{\text {Base }}<800 \mathrm{~nm}\right)$. So the gradient of the electron density is low, resulting in a low collector current caused by diffusion.

\section{Summary}

The Python package SyNumSeS has proven its capability for the simulation of various semiconductor devices. As these devices are also discussed in bachelor and master courses the package is well suited to support the lecture by visualizing physical quantities in the 


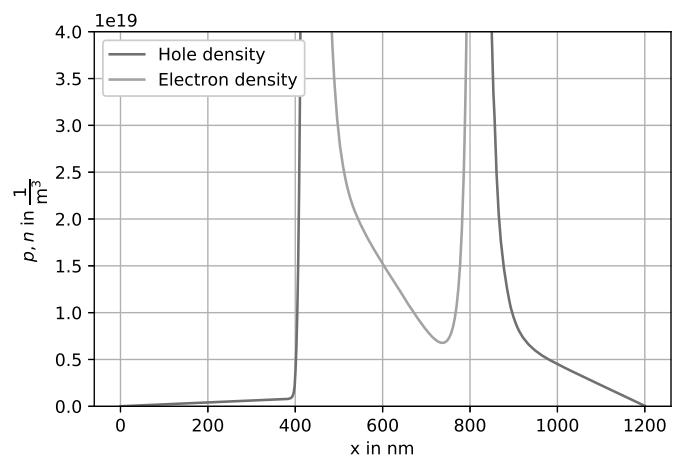

Figure 7: Electron and hole density across the bipolar transistor at saturation for $V_{\mathrm{CE}}=50 \mathrm{mV}$ and $V_{\mathrm{BE}}=0.6 \mathrm{~V}$.

device for different bias regions. Due to its fast simulation times it is also appropriate for lab courses where the students can investigate the influence of process and material parameters on the performance of a device.

This Python package can be installed by:

pip install synumses-pkg-pabele and cloned from

https://github.com/pabele/synumses with several examples using the JupyterLab notebook.

\section{References}

[1] Van Roosbroeck W. Theory of the Flow of Electrons and Holes in Germanium and Other Semiconductors. Bell System Technical Journal. 1950;29(4):560-607.

[2] Guyer JE, Wheeler D, Warren JA. FiPy: Partial Differential Equations with Python. Computing in Science \& Engineering. 2009;11(3):6-15.

[3] Scharfetter DL, Gummel HK. Large-signal analysis of a silicon Read diode oscillator. IEEE Transactions on Electron Devices. 1969;16(1):64-77.

[4] Farrell P, Rotundo N, Doan DH, Kantner M, Fuhrmann J, Koprucki T. Drift-Diffusion Models. In: Handbook of Optoelectronic Device Modeling and Simulation, edited by Piprek J, pp. 733-772. London, New York: CRC Press. 2017;

[5] Selberherr S. Analysis and Simulation of Semiconductor Devices. Wien, New York: Springer. 1984. 



\title{
Reducing Response Time with Data Farming and Machine Learning
}

\author{
Falk Stefan Pappert ${ }^{*}$, Oliver Rose \\ Fakultät für Informatik, Universität der Bundeswehr München, Werner-Heisenberg-Weg 39, \\ 85579 Neubiberg, Germany; *falk.pappert@unibw.de
}

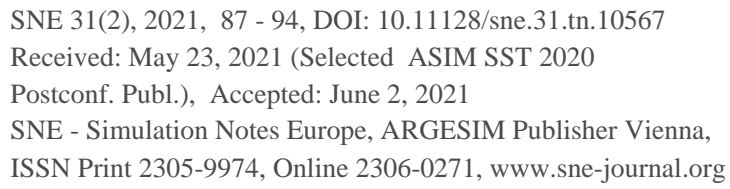

Abstract. In industry, there are numerous applications for simulation. However, simulation in our area usually takes some time even if a preexisting model just needs to be parameterized; there is still the run time, which will usually take at least a few minutes if not hours. In our current case, a planner wanted to know for a given product mix situation and for an equipment group with specific characteristics how much he can utilize the equipment without violating flow factor targets. A question, which arises several times during a typical workday as new orders are coming in and the situation on the shop floor is continuously changing. Since the user is usually asking the same question just with different parameters we are able to solve the waiting time problem while still giving good decision support. Instead of simulating every scenario at the time the user actually needs these answers, we use data farming to generate a large set of data points that are then used to train a neural network. This neural network then substitutes for the simulation and responds to the user immediately.

\section{Introduction}

A crucial task in modern industry is capacity planning. Robinson et al. [1] point out why accurate capacity planning is so important, yet so difficult to achieve in the highly sophisticated semiconductor industry. A planner faces numerous questions every day from short-term operative questions to long-term strategic ones. A necessary starting point to make any reasonable decsions is to know the available equipment capacity and how its' utilization influences the material flow.

As cycle times vary from product to product flow factors (cf. Equation 1) are good indictors to evaluate a production system.

$$
\text { flow factor }=\frac{\text { actual cycle time }}{\text { raw process time }}
$$

The trade off between utilization and flow factor can be visualized as operating curves (cf. [2]), which relate a system's flow factor against its utilization. Operating curves are an important tool in managing semiconductor fabs (cf. [3]).

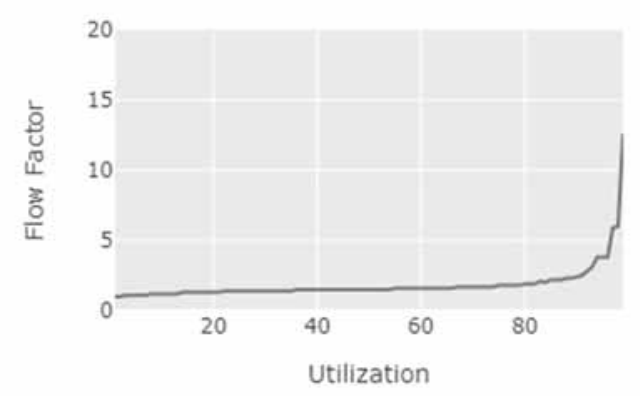

Figure 1: Operating Curve of a basic single equipment without any special features

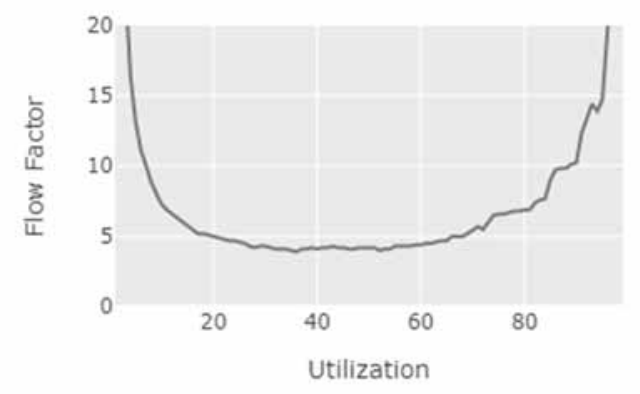

Figure 2: Operating Curve of a single batch-equipment with infrequent but long breakdowns

Examples of operating curves are shown in Figure 1 and Figure 2. Although these curves represent the behavior of the system at all utilization levels, usually not the whole operating curve is relevant to a planner.

What typically is of interest to our collegues is whether there is enough capacity for a given product mix or load. In modern days, this question has changed to 
whether it is possible to maintain a given flow factor with the given product mixes and loads. Therefore, it is important to know until which point an equipment group can be utilized before it violates flow factor targets. These thresholds are basically what we are looking for.

Figure 1 and Figure 2 also show that whether a system can handle a given material flow is not just based on its' utilization. Numerous factors are influencing equipment behavior; batching, breakdowns, and maintenance are just some examples. Based on these characteristics equipment groups are able to handle different utilization levels before reaching certain flow factors. As this differs from equipment to equipment this question needs to be answered often for different equipment groups.

It is the goal of our research to develop an approach that answers this question in a most timly fashion while still being sufficiently accurate to base planning and investment decisions upon. Traditionally, this is done at our industry partner with a calculation based on look up tables, which only included some factors. Although the look up is quite quick, the results were far from optimal since too few influencing factors were considered.

A typical solution approach would simply be to build or generate a simulation model for a given equipment group and run some simuation experiments. But this would still take some time, with large equipment groups maybe even a few minutes. Hence, this approach would not meet the response time requirement for the given problem. Byrne [4] proposed an approach to limit the number of necessary design points to calculate an operating curve. Althougth this would speed up simulation, there would still be some waiting time for results.

In the first section of this paper, we will give an introduction to the general idea behind our approach. In Section two, we will discuss some software development aspects of creating such a system. In the third section, we will briefly show the features considered in our current project. In the following sections, we will furthermore discuss the simulation model, data farming, and training of our artificial neural networks. In Section seven, we will shortly show our current results and give an outlook on our future plans in Section eight.

\section{System Overview}

As we have previously discussed, we aim to build a system which is able to quickly provide answers to the same question with changing parameters or configurations, which is repeatedly asked during a normal workday.
If these questions would only be asked from time to time or a response would not be that time critical a common approach would be to build a simulation model and answer the question after analyzing a simulation experiment.

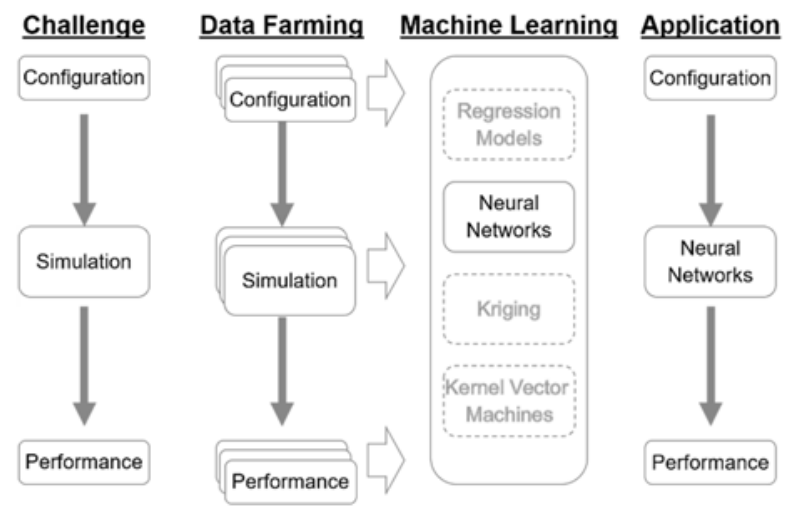

Figure 3: Generalization of discussed approach for a fast response system based on data farming and machine learning.

If short response times are very important, Figure 3 shows a generalized approach on how we answer this question. The challenge column would be the normal simulation experiment approach. The user has a question about a given system configuration. A simulation model representing this system configuration is built and its' performance is evaluated. After a reasonable number of simulation runs the user gets the answer.

As the simulation runs are the time consuming part we changed the system. Instead of creating or parameterizing a simulation model each time the user needs an answer to the question we move the simulation runs to a point in time long before the user asks our system. We use data farming to create the results to a huge number of possible factor combinations.

The resulting data set is then used as the supporting points for machine learing algorithms, in our case neural networks, to approximate a function that reproduces a response to a given configuration and therby replaces the simulation in the moment the user queries the system. Instead of directly asking for the results of a time-consuming simulation experiment, the user asks the neural network that is able to respond almost immediately.

Figure 4 shows a basic overview of our system. 


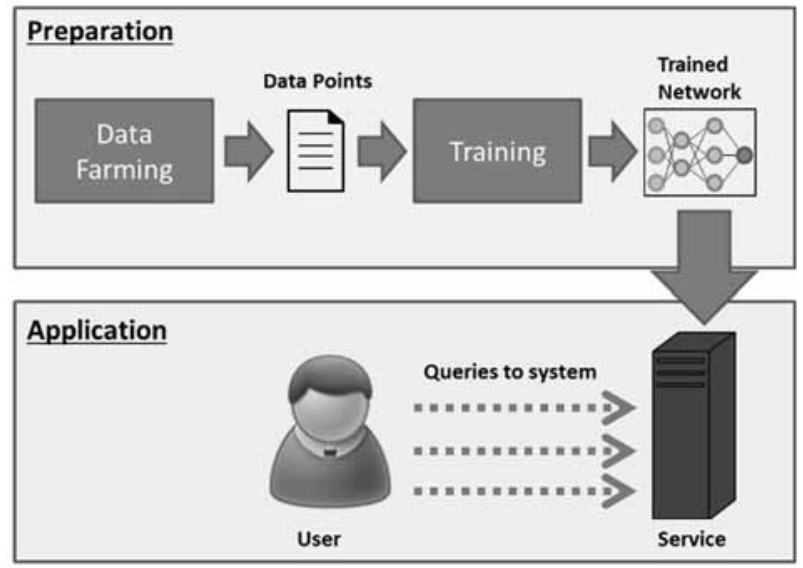

Figure 4: System overview.

\section{System Architecture}

In this section, we will discuss our system architecture from a software development point of view. We will start with the initial basic design and point our changes we have done to improve the system.

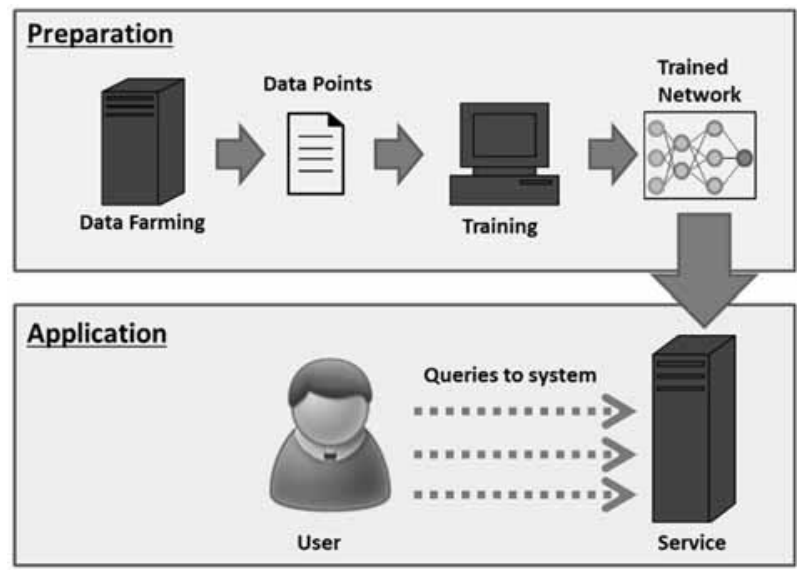

Figure 5: Basic system architecture.

Basing our architecture (cf. Figure 5) on the system overview shown in Figure 4 we planned for one big simulation-based data farming component, which would generate a huge set of supporting points. These data points would be transferred as a file to an R (cf. [5]) script handling data preparation and training of the neural network. With this setup, we were able to obtain reasonable results but we found that there is still a lot of room for improvement. One of our first and surprisingly valuable changes was a switch from using $\mathrm{R}$ to train our neural networks to Keras (cf. [6]). With R, depending on our data set, we were sometimes observing training times of a couple of days, which we attributed to reaching some memory boundaries.
We often had to abort after some time as no further progress was visible and it was hard to predict the remaining training time. However, even training times of a few hours considerably limit the amount of network configurations one can test in the hopes of improving results. The switch to Keras with an underlying Tensorflow (cf. [7]) library immediately improved training times incredibly. Furthermore, being able to utilize GPUs (graphics cards) for training improved training speeds to a point where instead of several hours or days we were looking at seconds and minutes for training. This new dimension of training times opened up the opportunity to consider neural architecture search to further improve the results of the neural network and thereby the whole system in the future.

A second big change to our system is the move from a monolithic piece of software to a service-based architecture using RESTful Web Services (cf. [8]). In this change, we see three major benefits to our system:

1. Ease of communication between system parts,

2. Scalability and distribution on multiple machines,

3. Replaceability of components.

In the beginning of the project, we made the conscious decision to implement different parts of our system with different languages. We see benefits in developing in Java with its object-oriented concept and type system paired with available IDEs supporting numerous ways of testing and debugging that make it very suitable to larger and more complex software projects. $\mathrm{R}$ on the other hand offered much easier access to mathematical functionality and neural networks. Nowadays, Python basically is the de facto standard language for data analyses and machine learning with a number of libraries and frameworks available and new systems usually being accessible only or at least first with Python.

Communication across these language barriers is often not easy with "direct calls". As most modern languages nowadays offer libraries, to easily implement web services, this is an elegant approach to handle communication between system parts written in different programming languages without much additional implementation overhead. Gone were complicated command line calls and file-based communications.

Most parts in our system can be quite computation intensive. While it is still reasonable to run small test cases on a single office PC, larger experiments benefit from good scalability and distribution. Furthermore, different parts of the system benefit differently from available hardware. 
While the simulation is mostly CPU and memory intensive, training neural networks significantly benefits from the availability of modern graphics cards. Being able to assign services to machines with the best fitting hardware is therefore another aspect, which is easily taken care of with services.

Besides distributing the different services on different machines, some services may in the future need additional computing power. With a service-oriented architecture, it is also easy to introduce load balancers which distribute requests of the same type on several machines offering the same service without any necessary changes to the client side. This makes setting up such a system very comfortable for different experimental environments.

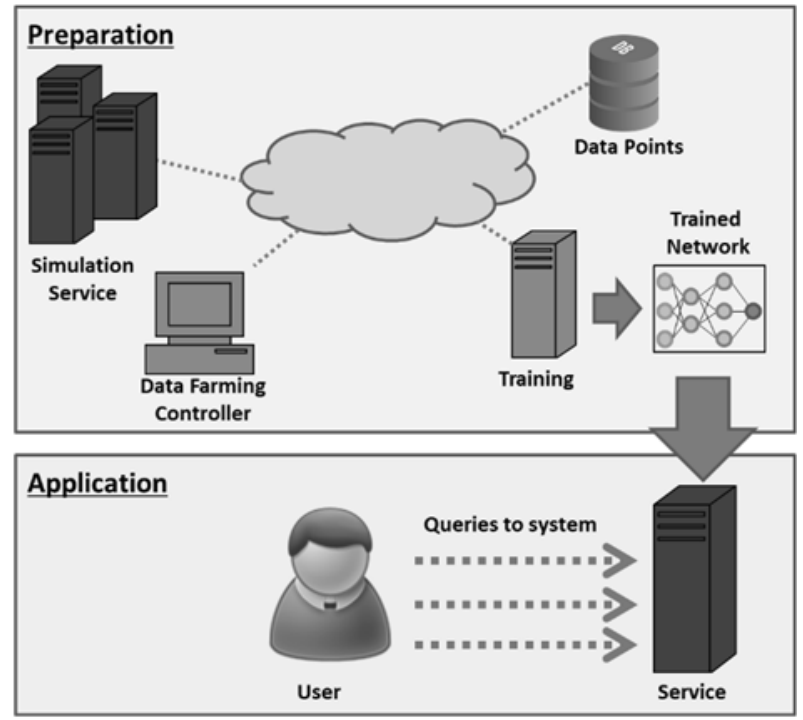

Figure 6: Service based system architecture.

Replaceability without the need to touch any other system component is also a great benefit. As we try different frameworks and approaches the current architecture offers us to simply replace some services while others stay the same. Changing the simulator, the persistence approach or even the machine learning technique are all simply done by putting the new component up as a service replacing the old one. Hence, with this change we gained a lot of scalability and flexibility for future experiments.

\section{Factors}

Starting with Robinson et al. [1] and Hopp and Spearmean [9] and a review of the previous planning methods we defined relevant features for our equipment group model.
Values for our factor levels were chosen based on a fab dataset from our industrial partner by looking for natural clusters and using representatives thereby capturing realistic workings points.

Most factors can be easily defined with single numerical values. These are shown as quantitative factors. Some factors shown as categorical in Table 1 represent more complex definitions. Product mix for example represents the number of different products as well as their percentage of the released material flow. For categorical features we selected three levels based on real equipment groups going from a low impact to a high impact setting with regard to the resulting flow factors.

\begin{tabular}{llll}
\hline Feature & Factor & \# & Type \\
\hline Batching & MaxBatch & 5 & Quant. \\
& MinBatchPercentage & 3 & Quant. \\
\hline Breakdown & MeanTimeBetweenFailure & 3 & Quant. \\
& BreakdownCapaLoss\% & 2 & Quant. \\
\hline Dedication & Dedication & 3 & Cat. \\
\hline Equipment \# & ToolCount & 7 & Quant. \\
\hline Maintenance & TimeToMaintenance & 3 & Quant. \\
& MaintCapaLos\% & 2 & Quant. \\
\hline Product Mix & ProductMix & 3 & Cat. \\
\hline Rework & ReworkPercentage & 3 & Quant. \\
\hline Process Time & RPT & 6 & Quant. \\
\hline Setup & SetupDuration & 3 & Quant. \\
\hline
\end{tabular}

Table 1: Feature and factor overview.

While initially considering only one factor per feature we now split some features into two factors for better scalability. This makes it easier for a future algorithm to generate new test points to validate and improve the resulting model. Additionally, the effect of some features is hard to capture with a single factor. For example, when considering two systems suffering from $25 \%$ loss of capacity due to breakdowns; one breaking down after 3 hours of productive time for about an hour while the other one runs fine for 3 weeks followed by a week of repair. We would expect the second system to perform worse with regard to cycle time and flow factor. Hence, we split breakdown into capacity loss and mean time between failures.

As the increase in factors brings a significant increase in design points considering a full design, we have not yet updated our training data set to include all new points. Although the training data set does not include all these points, we are still able to address them better and test for them. 


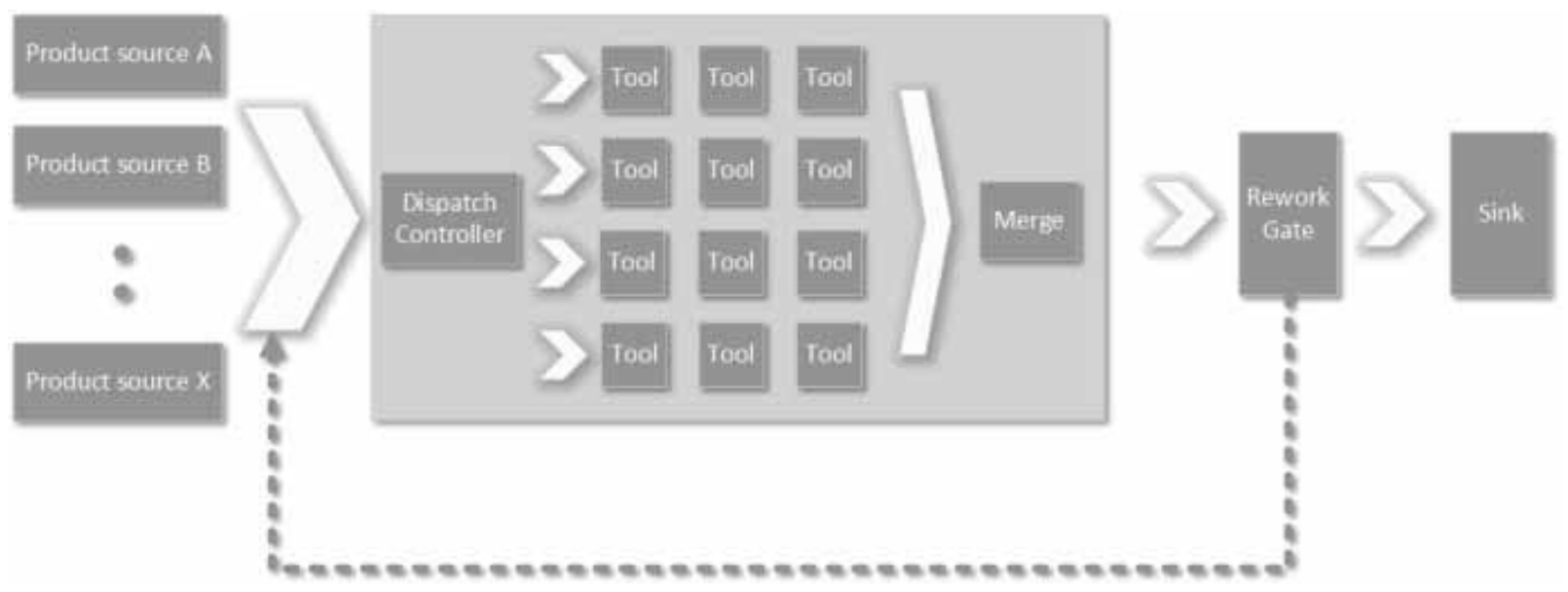

Figure 7: Simulation model structure.

\section{Evaluation and Simulation}

We use an inhouse developed factory simulator for all simulation runs in this project. As we have mentioned before the simulator is currently running as a service and simulation runs can be started by calling the service and handing over parameter values for each factor under consideration.

The simulation service will then automatically generate a simulation model based on the given parameters. Figure 7 shows a visualization of the equipment group model. As the goal of the simulation is to determine reasonable utilization values for the lowest flow factor possible and the location of defined flow factor threshholds, the next step is a static capacity analysis. This is necessary to run the simulation only with reasonable loading scenarios without wasting calculation time for extremely low utilization settings or incredibly overloaded systems. Based on the static capacity analysis we can now simply calculate the necessary lot releases to run the model at a specific utilization point.

We use a search strategy akin to binary search to look for the location with the lowest possible flowfactor. For each utilization point under evaluation simualtion runs are performed until the sample size for this point is determined to be large enough for a stable estimation. Flow factor thresholds are searched for similarly while reusing the results of previously tested plus new utilization points. Once the lowest flow factor value and all requested flow factor thresholds are determined the results are handed back.
Verification and validation are difficult when considering data farming, as it is almost impossible to evaluate every single simulation run. We deployed different strategies to ensure our simulation results reflect real world behaviour. The basis for this were unit tests to continuously check the simulation software during development. This was done to avoid unintended effects during programming. We additionally compared sample simulation results with the results from other simulation software packages. Additionally, we had a panel of experts reviewing results generated by the simulator and compare them to real factory data of equipment groups with similar characteristics. Of course, this cannot be done for all data points but helps to validate the system.

\section{Data Farming}

When considering the number of factors and factor levels, we are looking at a huge number of data points to evaluate. In addition to all these data points, we are also looking at several simulation runs per data point. As we have mentioned before each data point is determined by calculating several utilization points for which we run a number of simulations each. Not all utilization points take the same amount of repetitions as we determine this number on the fly during the evaluation.

After simulating an initial set of replications, we calculate the confidence interval half-length and mean. Then, we compare their quotient with the relative error we aim for. If the quotient is still larger than the relative error, we run another set of replications. 
We repeat this until the relative error is smaller than the quotient (cf. [10]).

On average, we ran about 825 simulations for a single data point to determine the location of the lowest flow factor value and three thresholds. Considering even just one factor per feature we were looking at almost 460000 data points which total in almost 380 million simulation runs just to generate the supporting points for our project.

Although the evaluation of single data points is feasible on a normal PC, running these almost 460000 of data points on one of our simulation servers took several weeks. With our change in architecture and therefore much better scalability, we hope any future additions to our current data set will be available much faster.

\section{Training}

With all these data points from simulation, we were still only looking at the supporting points for our system. As we have mentioned before we moved from using $\mathrm{R}$ to Keras to implement the training of our neural networks which considerably increased training speed and made it much easier to test different layer configurations. Typically, we aim to minimize the mean squared error (MSE) of our testset. When trying to evaluate the usefulness of any trained network we additionally present the predicted results graphically.

Figures 8 to 10 show the results of network configuration and training parameter sets we tested. The diagrams are historgrams of how successful the predictions have been. Starting from the left results are grouped by the error in prediction compared to the simulation result. The first bar represents less than $1 \%$ distance and each following bar an additional 1\%. E.g., a scenario for which the network predicted 75.5 but the simulation estimated 73 would fall into the third bin. Please be aware that the $\mathrm{x}$-axis in Figure 8 is using a different range from the other shown results. We chose to do this to be able to show the set of extremly poor predictions that is not present in the other results.

Although the quality of the trained networks can differ greatly between network configurations, all of the results shown here were able to reduce the MSE continuously during training and on a first glance seemed to work quite well. Only when visualizing what the results meant with regard to the actual problem at hand, it became obvious that some of these networks are not usefull at all to solve our problem.

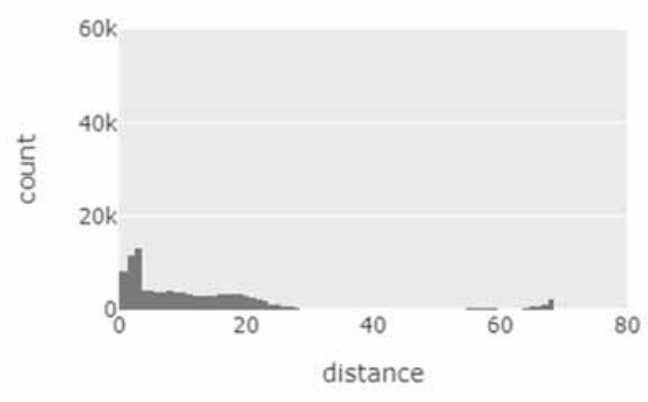

Figure 8: Visualization of the distance between predicted and simulated value; example 1 .

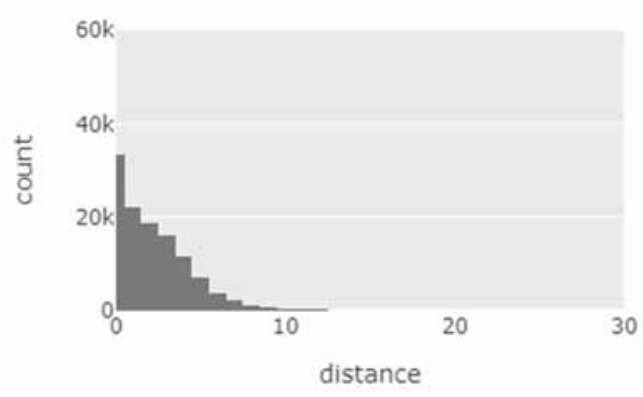

Figure 9: Visualization of the distance between predicted and simulated value; example 2 .

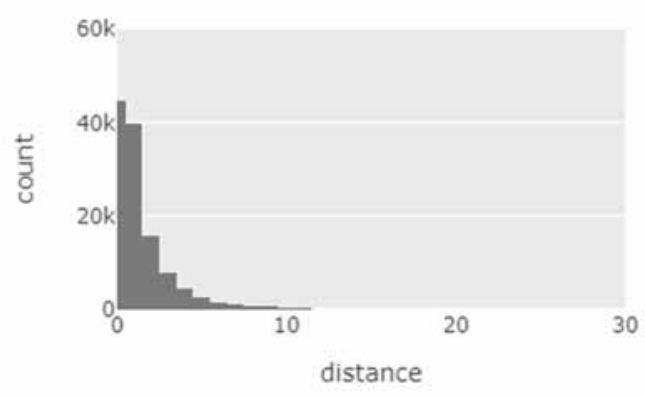

Figure 10: Visualization of the distance between predicted and simulated value; example 3.

Besides network architecture we found that training parameters like batch sizes and the number of episodes have a significant impact on result quality. In fact, Figures 8 and 9 are based on the same network configuretion but used different batch parameters for training. 


\section{Results}

We set out to achieve two objectives. First, we wanted to create a system, which is able to respond immediately to a user query. Second, we needed to have sufficiently good results to base planning and investment decisions upon.

On the first objective, we are where we want to be. The application server running on a better office PC with a modern graphics card responds within $300 \mathrm{~ms}$ to a user query. The majority of this time is actually spent on allocating the graphics card. Running the network for the prediction just on the CPU without GPU support this response time could actually be reduced even more as predicting is quite fast with just the CPU.

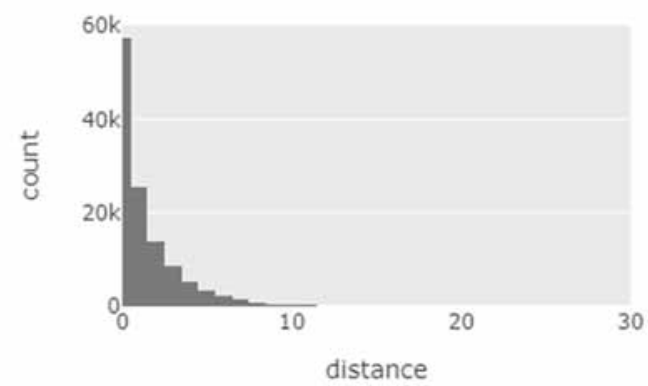

Figure 11: Visualization of the distance between predicted and simulated value; current results.

Looking at the second objective, Figure 11 shows our currently best results derived just with manual testing of different network architectures and training parameters. About $80 \%$ of our test points are predicted with an error of less than $3 \%$. Almost all datapoints are predicted with less then $10 \%$ error from our simulation results.

Furthermore, we are no longer seeing any artifacts as in Figure 8. Althoug these are reasonably good results and a prediction quality of $3 \%$ or better for a majority of data points would be good enough to base planning on these numbers, we are still looking at $20 \%$ of predictions being off by up to and $10 \%$. Considering high utilization scenarios overestimating possible utilization by $10 \%$ error could have a serious impact on the performance of the material flow and ability to maintain promised delivery dates.

On the other hand, underestimating utilization thresholds by $10 \%$ would mean significant loss of production capacity or triggering an investment in equipment before it is actually necessary. We therefore still see some need for improvement.

\section{Summary and Outlook}

In this paper, we presented our approach to create a system with minimal response time to a user query, which we would usually answer by simulation. We discussed some aspects of software architecture to improve scalability and flexibility of the software. The presented system uses a simulation model to do data farming for supporting points, which are then presented as training data set to machine learning methods like neural networks. The resulting trained system is able to respond to the user queries within moments.

Although the system already works as a proof of concept, the accuracy is still not where it would need to be to be applicable in an industrial setting. We are working on two approaches to improve prediction quality for all points.

First, we have seen during our manual configuration of the the neural network that network architecture and training parameters tend to have a big impact on result quality. With our improved training speeds, we are planning to improve prediction quality by automating the process of finding a good network configuration. There are several promising approaches to do this. We are currently working on adding a neuroevolution (A broader explanation can be found in [11]) service to our system. As an alternative, we are also looking at Auto-Keras (cf. [12]).

The second approach targets the somewhat infrequent supporting points within our training data set. Since adding additional levels to factors drastically increase the number of total points to calculate for a full design, simply adding more levels would be a very computation intensive approach.

Instead, we are seeking to improve the quality of the systems response by automatically searching for points with bad predictions and adding additional supporting points near those points to our training data set. Idealy, this would improve prediction quality in those areas and therefore for the whole system.

\section{Ackowledgements}

We would like to thank Dr Thomas Mayer for many interesting discussions on system architecture and machine learning. 


\section{References}

[1] Robinson J K, Fowler J, Neacy E. Capacity Loss Factors in Semiconductor Manufacturing. FabTime Inc 2003 https://www.fabtime.com/files/CapPlan.pdf.

[2] Aurand S, Miller P. The operating curve: a method to measure and benchmark manufacturing line productivity. 1997 IEEE/SEMI Advanced Semiconductor Manufacturing Conference and Workshop ASMC 97 Proceedings. 1997 IEEE/SEMI Advanced Semiconductor Manufacturing Conference and Workshop; 1997 Sep; Cambridge, MA, USA. IEEE. 391-397. doi: 10.1109/ASMC.1997.630768.

[3] Fayed A, Dunnigan B. Characterizing the Operating Curve - how can semiconductor fabs grade themselves?.. 2007 International Symposium on Semiconductor Manufacturing; 2007 Oct; Santa Clara, CA, USA. Place of Publication: publisher. 1-4. doi: 10.1109/ISSM.2007.4446827.

[4] Byrne N M. A framework for generating operational characteristic curves for semiconductor manufacturing systems using flexible and reusable discrete event simulations [dissertation]. School of Mechanical and Manufacturing Engineering. Dublin City University; 2012.

[5] Verzani J. Using R for Introductory Statistics. $2^{\text {nd }}$ Edition. New York, USA: CRC Press; 2014.518p

[6] Géron A. Hands-On Machine Learning with Scikit-Learn, Keras, and TensorFlow: Concepts, Tools, and Techniques to Build Intelligent Systems. $2^{\text {nd }}$ Edition. Sebastopol: O'Reilly; 2019.600p
[7] Abadi M, Barham P, et al. TensorFlow: A System for Large-Scale Machine Learning. Proceedings of the 12th USENIX Symposium on Operating Systems Design and Implementation (OSDI '16). 12th USENIX Symposium on Operating Systems Design and Implementation (OSDI '16); 2016 Nov; Savannah, GA, USA. 265-283. ISBN: 978-1-931971-33-1.

[8] Fielding R T. Architectural Styles and the Design of Network-based Software Architectures [dissertation]. University of California, Irvine; 2000.

[9] Hopp W J, Spearman M L. Factory Physics. $3^{\text {rd }}$ Edition. New York: McGraw-Hill; 2008.

[10] Law A M, Kelton W D. Simulation Modeling and Analysis. $3^{\text {rd }}$ Edition. New York: McGraw-Hill; 2000. 760p

[11] Stanley K O, Clune J, Lehman J. et al. Designing neural networks through neuroevolution.

Nat Mach Intell. 2019; 1: 24-35. doi: 10.1038/s42256-018-0006-z.

[12] Jin H, Song Q, Hu X. Auto-Keras: An Efficient Neural Architecture Search System. Proceedings of the 25th ACM SIGKDD International Conference on Knowledge Discovery \& Data Mining. 25th ACM SIGKDD International Conference on Knowledge Discovery \& Data Mining; 2019; Anchorage, AK, USA, NY, USA: Association for Computing Machinery. 1946-1956. doi: $10.1145 / 3292500.3330648$. 


\title{
Lifetime Modelling of Electrical Machines using the Methodology of Design of Experiments
}

\author{
Lucas Vincent Hanisch*, Markus Henke \\ Inst. for Electrical Machines, Tractions and Drives, Technische Universität Braunschweig, Hans-Sommer Str. 66, \\ 38118 Braunschweig, Germany; *I-v.hanisch@tu-braunschweig.de
}

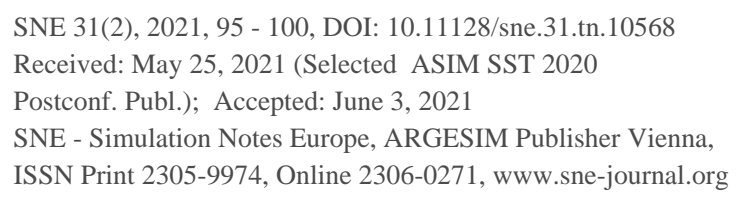

SNE 31(2), 2021, 95 - 100, DOI: 10.11128/sne.31.tn.10568

Received: May 25, 2021 (Selected ASIM SST 2020

Postconf. Publ.); Accepted: June 3, 2021

SNE - Simulation Notes Europe, ARGESIM Publisher Vienna,

ISSN Print 2305-9974, Online 2306-0271, www.sne-journal.org

Abstract. In the coming years, electromobility will be confronted with increasing demands regarding the reliability of electrical machines. In this paper a modeling methodology is presented, which allows to estimate the reliability and lifetime of the insulation system of electrical machines. Different statistical and physical modeling methods are presented, which are transformed for the later multiple regression. The methodology of Design of Experiments (DoE) is used to describe the insulation system. Since the effort for the experimental design of the DoE varies strongly with the number of effects to be investigated and the statistical accuracy, different experimental designs are presented, which can be considered for different numbers of factors. Depending on the research question, a suitable experimental design can be selected. For the calculation of the lifetime, Miner's rule is used in addition to the multiple regression, so that the percentage lifetime consumption due to a load spectrum can also be calculated.

\section{Introduction}

In the future, technologies such as electrified aircraft, trolley wire trucks or autonomous driving will be established in the field of electric mobility. Reliability, durability and safety are important criteria for the acceptance of new technologies in society. In addition to increased safety requirements, these technologies must also be able to cope with new, more challenging boundary conditions.

Whereas bearing damage used to be the most frequent cause of failure of electrical machines, the increased requirements and new boundary conditions lead to a more varied error pattern [1], [2]. A deep understanding of the causes of failure and the relevant damage mechanisms is necessary to design electrical machines for these new applications.
In addition to the design of durable machines that operate under increased environmental conditions, lifetime models, on the other hand, can help electrical machines achieve a minimum target lifetime for a given load. This offers potential to save resources and reduce costs. Lifetime models and reliability analyses are therefore becoming increasingly important in the design process of electrical machines.

\section{Damage Mechanisms}

The damage of electrical machines is caused by various mechanisms from different physical disciplines and there are various modelling approaches to model these damage processes. Since the use of wide bandgap semiconductors and the increasing electrical load, the cause of failure of electrical machines is increasingly based on faults and breakdowns of the electrical insulation system [2]. Because failures of the electrical insulation system will occure more frequently in the future, research concentrates on lifetime models of these insulation systems.

Basically, with regard to the aging effects that damage the insulation system, a distinction can be made between constant stresses and transient stresses. The probability of a fault in the insulation system at constant stresses is proportional to the number of operating hours and at transient stresses proportional to the number of transient effects. Figure 1 shows as examples for constant stresses the ambient temperature and the voltage slope oval and for transient stresses partial discharges rectangular.

A breakdown in the insulation system often occurs due to different combinations of the individual stresses or the interactions of these stresses.

As an example for the interaction of stresses Figure 1 shows the interaction between the temperature and the voltage slope. Insulating materials heat up due to dielectric losses. The dielectric power loss as well as the dielectric loss factor are again frequency dependent. Power electronic signals with high voltage slope and high frequency harmonics can therefore influence the temperature of the insulation system. 


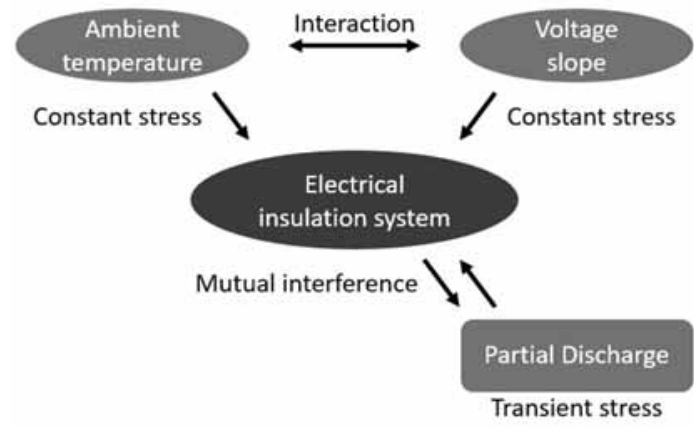

Figure 1: Examples of different stresses and their interactions.

An interaction can also become effective by changing the properties of the insulation system. Partial discharges can damage the insulation system and lead to air-filled cavities. Due to the low permittivity of air and the significantly lower dielectric strength of air compared to insulation materials, increased field strengths in these cavities can intensify partial discharges and the aging of the insulation system. In [3] an interaction due to changed properties of the insulation system is called indirect interaction.

\section{Modeling Methodologies}

The modelling approaches can be divided into two different categories - physical and the statistical approaches.

\subsection{Physical modeling approaches}

The goal of physical modelling approaches is the mathematical description of the aging effects. A holistic model, in which the different aging mechanisms from different physical disciplines are integrated, is very complex. In addition, at certain activation energies additional phenomena occur, become more dominant or the damage effect has to be described mathematically completely different.

The advantage compared to statistical models is that no lifetime tests are necessary for the parameterization of the model. Only the measurement of single physical quantities is necessary.

One of the first physical models to describe aging effects was developed by Crine and was originally used for extrudet dielectric cables. Crine takes up Artbauer's theory and assumes that the dielectric strength of amorphous insulation materials is essentially determined by the presence of vacancies induced by electromechanical deformation of molecular chains in the insulation material. In these vacancies, the free electrons find more favourable conditions to absorb the energy necessary for impact ionization due to the applied electric field [4]. Crine assumes that above a critical field strength, the number of these vacancies increases and they combine to form larger submicrocavities.
When submicrocavities have formed, electrons or ions are strongly accelerated under the influence of the electric field and can absorb enough kinetic energy to break weak Van der Waals bonds. As the submicrocavities expand, the electrons absorb even more energy and break more molecular bonds until the insulating material finally collapses and electrical breakdown occurs [5].

Crine describes the probability of breaking Van der Waals bonds depending on temperature $T$ and electric field strength $E$ as follows:

$$
p^{+}(T, E) \cong \frac{k T}{h} \exp \left(-\frac{\Delta G-e \lambda E}{k T}\right)
$$

Here $k$ is the Boltzmann constant, $h$ the Planck's constant, $\Delta G$ the critical field strength, $e$ the charge of an electron and $\delta$ the length of the free path. Additionally Crine considers the probability of the backward process as

$$
p^{-}(T, E) \cong \frac{k T}{h} \exp \left(-\frac{\Delta G+e \lambda E}{k T}\right)
$$

The net destruction rate is the subtraction of these probabilities

$$
p=p^{+}-p^{-} \cong \frac{2 k T}{h} \exp \left(-\frac{\Delta G}{k T}\right) \sinh \left(\frac{e \delta E}{k T}\right)
$$

In Crines physical model, the lifetime of the insulation system is given as the reciprocal of the destruction rate

$$
L \cong \frac{h}{2 k T} \exp \left(\frac{\Delta G}{k T}\right) \operatorname{csch}\left(\frac{e \lambda E}{k T}\right)
$$

At high fields, equation (4) reduces to

$$
L \cong \frac{h}{2 k T} \exp \left(\frac{\Delta G-e \lambda E}{k T}\right)
$$

After Crine, Lewis developed a new physical model that is also based on the formation of microcavities and the breaking of molecular bonds. Just like Crine, Lewis also takes into account the temperature $T$ and the electric field strength $E$. The destruction rate $K_{\mathrm{b}}$ and the formation rate $K_{\mathrm{r}}$ of the bonds are determined as follows:

$$
\begin{gathered}
K_{b}(T, E)=\frac{k T}{h} \exp \left(-\frac{U_{b}-\gamma_{b} \varepsilon E^{2}}{k T}\right) \\
K_{r}(T, E)=\frac{k T}{h} \exp \left(-\frac{U_{r}+\gamma_{r} \varepsilon E^{2}}{k T}\right)
\end{gathered}
$$

$U_{\mathrm{b}}$ and $U_{\mathrm{r}}$ are the critical energies at which the bonds break or form again. $\varepsilon$ is the dielectric permittivity and $\gamma_{\mathrm{b}}$ or $\gamma_{\mathrm{r}}$ are fitting parameters with the dimensions of a volume. The aging of the insulation system is equated with the propagantion of cracks and voids in the insulation system according to the Griffith criterion [6]

$$
L=\int_{0}^{b_{c}} \frac{1}{K_{b}(1-b)-K_{r} b} d b
$$

$b_{\text {c }}$ represents the critical number of broken molecular bonds above which insulation failure occurs. 


\subsection{Statistical modeling approaches}

In contrast to physical models, statistical lifetime models are based on accelerated lifetime tests. The most popular statistical lifetime model was developed by Arrhenius and describes the quantitative dependence of the chemical reaction rate $r$ on the temperature $T$

$$
r=A \cdot e^{-\frac{E_{A}}{k T}}
$$

where $A$ is a constant and $E_{\mathrm{A}}$ is the activation Energy. Dakin used Arrhenius' equation to describe the processes taking place in the insulation material as a function of temperature. Except for the constants Dakin uses the same equation as Arrhenius [7]:

$$
L=A_{\text {Dakin }} \cdot e^{\frac{B_{\text {Dakin }}}{T}}
$$

Partial discharges are often cited in the literature as the cause of electrical aging of electrical insulation systems. Their frequency and effects on service life increase exponentially with increasing voltage $V$. Mathematically, this realationship can be described using the inverse power model

$$
L=c \cdot V^{-n}
$$

here $c>0$ is a material constant and $n>0$ is the power law constant.

\section{Design of Experiments}

As mentioned before, the parameterization of the models from chapter 2.2 is done by accelerated lifetime tests. Despite the increased conditions compared to real operation, these tests are complex and time consuming. Physical models, on the other hand, are mathematically very complex and often only consider the electric field strength and temperature. The physical modelling of additional aging effects and the modelling of the individual interactions leads to an exponential increase in complexity and to the failure of the purely physical modelling approach. With the methodology of Design of Experiments (DoE) it is possible to model many effects including their interactions and to reduce the number of necessary life tests to a minimum.

The basic idea is that the insulation system, or even any deterministic system, can be described with a mathematical model. Different approaches can be used as mathematical models. Some were presented in chapter 2 . The parameterization of the model is done after the complete evaluation of the experimental design. DoE is therefore one of the statistical model approaches. In the following different experimental designs are discussed.

\subsection{Full factorial design}

In an experimental design, the number of effects to be investigated, the number of measuring points and the number of measured values per measuring point are defined. The advantage of an experimental design compared to a one-factor-at-a-time-plan is that each test result can be used to calculate several effects. This considerably reduces the test effort.

If, for example, eight measured values per measureing point are necessary to determine the influence of a factor on the service life of the insulation system with acceptable accuracy, a "one factor at a time" plan requires 32 tests. In the first 8 tests the lifetime would be determined for the factors $x_{1}, x_{2}$ and $x_{3}$ at a low level. Then eight tests would be carried out on each factor at an increased level to investigate the influence of a factor on the service life. This is shown in figure $2 \mathrm{a}$. With a full factorial design, 16 tests would be sufficient to determine the effect of a factor with the same accuracy. Instead of examining the effect once by comparing eight value pairs, the effect is examined four times by comparing two value pairs. The number of value pairs used to calculate an effect and the statistical validation are identical. Figure $2 b$ shows the full factorial design. Since the test specimens can only be used once in the accelerated lifetime tests, the full factorial design reduces costs and time. In addition, the information content obtained from the measurements is increased, because the effect of the factors can be analyzed at different levels.

With each additional factor added to an experimental design, the information content of the DoE increases. With increasing information content, the effort of the experimental design increases with $F^{\mathrm{m}} . F$ is the number of measuring points in one dimension and $m$ the number of factors. The experimental design in figure $2 b$ has $2^{3}=8$ measuring points. The number of measurements per measuring point determines the accuracy of the experimental design. The experimental design in figure $2 \mathrm{~b}$ has a total of 16 measurement values with which the influence of the effects on the service life of the insulation system can be determined.
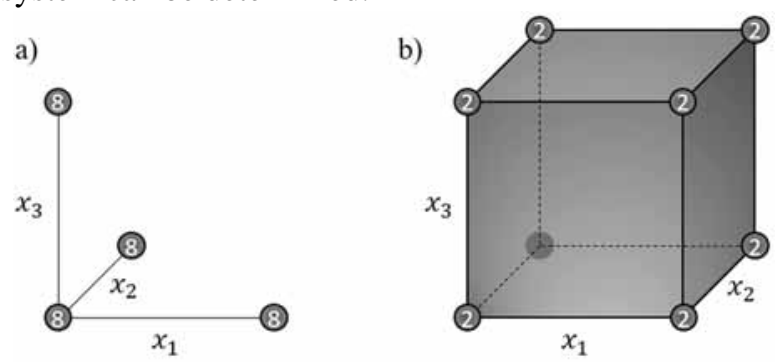

Figure 2: Comparison of a "one factor at a time" plan a) with a full factorial design b).

SNE 31(2) - 6/2021 


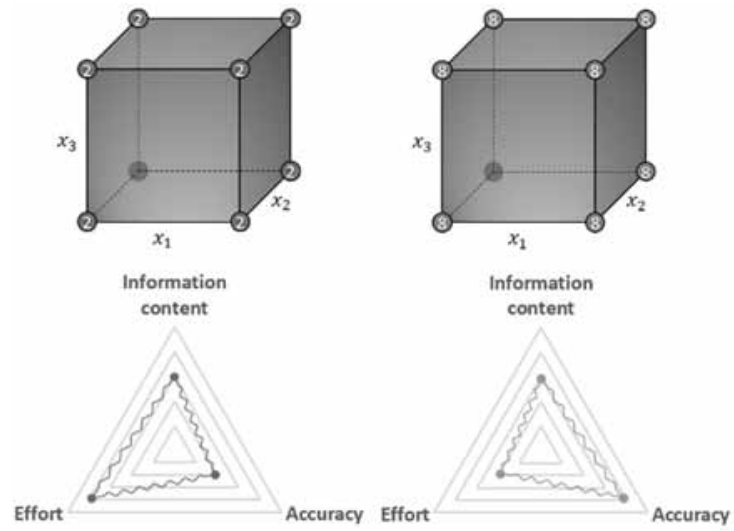

Figure 3: Variation of an experimental design by an increased number of tests.

If the number of tests per measurement point is increased from two to eight, 64 measurement values can be used. The accuracy increases, but also the effort. Information content, accuracy and effort of an experimental design are inseparably linked. The optimal experimental design must be adapted to the respective requirements. Figure 3 shows the combination of the three basic properties of an experimental design. If the point is further outside the triangle, an increase in information, an increase in accuracy or a reduction in effort is meant.

With statistical models there is always the risk of errors. There are two possible types of errors that can occur. The influence of a factor on the aging of the insulation system could be assumed to be significant, although in reality it is not responsible for the aging or a significant effect on the aging of an insulation system could be overlooked. In a hypothesis test these errors are called first type errors and second type errors. In order to determine the number of test specimens required in a design for a given accuracy, such a hypothesis test should be performed.

The hypothesis is as follows: The factor has no effect on the lifetime of the insulation system. In table 1 the four possible decisions of this hypothesis test are shown. The accuracy of the experimental design is determined by the errors of the first $\alpha$ and second type 6 and the change in lifetime $\Delta L$ to be detected. Table 2 shows the number of tests to achieve the desired accuracy.

\begin{tabular}{c|c|c|}
\multicolumn{1}{c}{} & \multicolumn{1}{c}{ Hypothesis correct } & \multicolumn{1}{c|}{ Hypothesis wrong } \\
\cline { 2 - 3 } $\begin{array}{c}\text { Assume } \\
\text { hypothesis }\end{array}$ & $\begin{array}{c}\text { Non-significant effect } \\
\text { detected }\end{array}$ & $\begin{array}{c}\text { Type I error } \alpha \\
\text { Significant effect not } \\
\text { recognized }\end{array}$ \\
\cline { 2 - 3 } $\begin{array}{c}\text { Reject } \\
\text { hypothesis }\end{array}$ & $\begin{array}{c}\text { Type II error } \beta \\
\text { non-significant effect } \\
\text { assumed to be significant }\end{array}$ & $\begin{array}{c}\text { Power }(1-\beta) \\
\text { Significant effect detected }\end{array}$ \\
\cline { 2 - 3 } & &
\end{tabular}

Table 1: Possibilities of a hypothesis test.

\begin{tabular}{|c|c|c|c|c|c|c|c|c|c|c|c|c|c|c|c|}
\cline { 2 - 15 } \multicolumn{1}{c|}{$\alpha=10 \%$} & \multicolumn{4}{c|}{$\alpha=5 \%$} & \multicolumn{4}{c|}{$\alpha=1 \%$} & Type lerror \\
\hline$\Delta \mathbf{L} / \sigma$ & $60 \%$ & $70 \%$ & $80 \%$ & $90 \%$ & $60 \%$ & $70 \%$ & $80 \%$ & $90 \%$ & $60 \%$ & $70 \%$ & $80 \%$ & $90 \%$ & Power \\
\hline 0,5 & 60 & 78 & 102 & 140 & 82 & 102 & 128 & 172 & 132 & 158 & 192 & 242 & \\
\hline 0,75 & 28 & 36 & 46 & 64 & 38 & 46 & 58 & 78 & 62 & 72 & 88 & 110 & \\
\hline 1 & 16 & 22 & 28 & 36 & 22 & 28 & 34 & 46 & 36 & 42 & 52 & 64 & \\
\hline 1,5 & 10 & 12 & 14 & 18 & 12 & 14 & 18 & 22 & 18 & 22 & 26 & 30 & \\
\hline 2 & 6 & 8 & 8 & 12 & 8 & 10 & 12 & 14 & 12 & 14 & 16 & 20 \\
\hline
\end{tabular}

Table 2: Necessary number of tests for a certain accuracy when each factor has two levels.

If a significant effect is to be detected at $(1-\beta)=90 \%$, if it changes the lifetime by at least one standard deviation $\sigma$ on average, and a non-significant effect is to be falsely assumed to be significant only at $\alpha=1 \%, 64$ tests are required for this experimental design. This corresponds to the experimental design shown in figure 3 on the right. The experimental design in figure 3 left is less complex with 16 tests, but a significant effect is detected only at $(1-\beta)=60 \%$ and the probability of erroneously assuming a non-significant effect as significant is $\alpha=10 \%$. Since the standard deviation $\sigma$ influences the accuracy of the DoE, the test specimens should be manufactured as identically as possible and the accelerated lifetime tests should be performed under the same boundary conditions.

\subsection{Fractional factorial design}

Not only a high accuracy requires a high number of tests but also with increasing information content and the consideration of further influencing factors the effort of an experimental design increases with $F^{\mathrm{m}}$. One possibility to efficiently investigate several effects despite the exponentially growing effort is the use of fractional factorial designs. The correlation between information content, accuracy and effort from figure 3 can not be avoided, but in experimental designs that take many factors into account, some information is irrelevant or can be excluded in advance as a cause for the aging of the insulation system. This is especially true for the $i$-fold interactions between the factors $m$, which can be calculated as follows

$$
\left(\begin{array}{c}
m \\
i
\end{array}\right)=\frac{m !}{i ! \cdot(m-i) !}
$$

The larger $m$ the more interactions are predominantly investigated.

To investigate the effect of a factor on the lifetime of the insulation system is the primary goal of the lifetime model. Also the effect of the interaction of two factors on the lifetime is of interest. The influence of higher interactions is physically difficult to assess and often negligible compared to two-fold interactionsor simple effects. Instead of these higher interactions additional single or double interactions could be investigated. In the case of fractional factorial designs not all measureing points are executed. Figure 4 shows the comparison of full factorial design and a fractional factorial design. 
a)

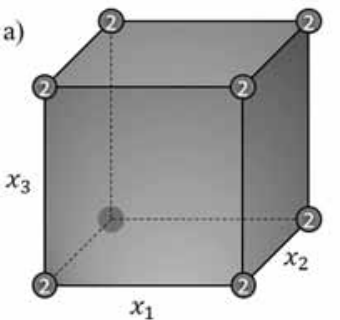

b)

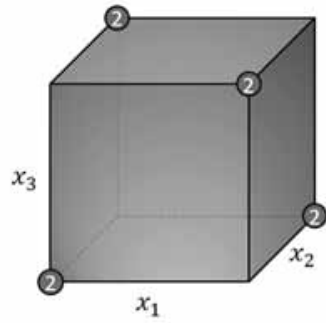

Figure 4: Comparison of a full factorial design a) and a fractional factorial design b).

This leads to a loss of information and to the fact that individual effects mix with each other. It is later not possible to distinguish which of these mixed effects is responsible for the aging of the insulation system. However, if simple factors and two-fold interactions are mixed exclusively with higher interactions whose effect on the lifetime is negligible, the question of how to distinguish between these effects is unnecessary. Table 3 lists and evaluates various fractional factorial designs.

\section{Lifetime Modeling}

After different modelling methods were described in chapter 2 and the DoE was described in Chapter 3, this chapter focuses on the development of the lifetime model based on the DoE.

\subsection{Multiple regression}

As a mathematical model to describe the insulation system, multiple regression is used. With this model it is possible to adapt the relationship between the influencing variables $x_{\mathrm{i}}$ and the lifetime as target variable $y$ to the measured values. For the description a full factorial design with two measured values per measuring point is used, as shown in figure $4 \mathrm{a}$. The formula for the calculation of the lifetime is given with the multiple regression as follows

$$
\begin{aligned}
y= & z_{0}+z_{1} x_{1}+z_{2} x_{2}+z_{3} x_{3}+z_{12} x_{12}+ \\
& z_{13} x_{13}+z_{23} x_{23}+z_{123} x_{123}
\end{aligned}
$$

where $z_{\mathrm{i}}$ is the influence of factor $x_{\mathrm{i}}$. The measured values required to parameterize equation (13) are designated $c_{\mathrm{i}}$ in table 4 . In the experimental design in figure $4 \mathrm{a}$, each factor has two levels, designated by the values -1 for the lower level and 1 for the higher level in table 4 . For two measured values, the mean value could also be used instead of the expected value. If there are several measured values, the expected value should be used. The measured values in life cycle investigation are usually Weibull distributed.

To determine the influence of the factors $z_{i}$ on the lifetime of the insulation system, equation (13) can be converted to matrix notation:

$$
\underline{Z}=\underline{X}^{-1} \cdot \underline{Y}_{i}
$$

$\underline{X}$ and $Y_{i}$ are shown in green and blue in Table 4. The relation of the individual factors with the lifetime of the insulation system is not linear but can be described with the models from chapter two. These non-linear correlations must be transformed and used in the linear equation (13). In equation (15) this is represented for three factors which were described with the statistical approaches from chapter 2.2

\begin{tabular}{|c|c|c|c|c|c|c|c|c|c|c|c|}
\hline $\begin{array}{c}\text { Measuring } \\
\text { points }\end{array}$ & Constant & & & $x_{3}$ & $x_{12}$ & $x_{13}$ & $x_{23}$ & $x_{123}$ & $\begin{array}{c}\text { Measured } \\
\text { value 1 }\end{array}$ & $\begin{array}{c}\text { Measured } \\
\text { value } 2 \\
\end{array}$ & $\begin{array}{c}\text { Expected } \\
\text { value }\end{array}$ \\
\hline 1 & 1 & -1 & -1 & -1 & 1 & 1 & 1 & -1 & $c_{11}$ & $c_{12}$ & $y_{1}$ \\
\hline 2 & 1 & -1 & -1 & 1 & 1 & -1 & -1 & 1 & $c_{21}$ & $c_{22}$ & $y_{2}$ \\
\hline 3 & 1 & -1 & 1 & -1 & -1 & 1 & -1 & 1 & $c_{31}$ & $c_{32}$ & $y_{3}$ \\
\hline 4 & 1 & -1 & 1 & 1 & -1 & -1 & 1 & -1 & $c_{41}$ & $c_{42}$ & $y_{4}$ \\
\hline 5 & 1 & 1 & -1 & -1 & -1 & -1 & 1 & 1 & $c_{51}$ & $c_{52}$ & $y_{5}$ \\
\hline 6 & 1 & 1 & -1 & 1 & -1 & 1 & -1 & -1 & $c_{61}$ & $c_{62}$ & $y_{6}$ \\
\hline 7 & 1 & 1 & 1 & -1 & 1 & -1 & -1 & -1 & $c_{71}$ & $c_{72}$ & $y_{7}$ \\
\hline 8 & 1 & 1 & 1 & 1 & 1 & 1 & 1 & 1 & $c_{81}$ & $c_{82}$ & $y_{8}$ \\
\hline
\end{tabular}

$$
\begin{aligned}
\log y= & z_{0}+z_{1} \cdot e^{B_{\text {Dakin }} \cdot x_{1}}+z_{2} \cdot \log x_{2}+ \\
& z_{3} \cdot \log x_{3}+z_{12} \cdot e^{B_{\text {Dakin }} \cdot x_{1}} \cdot \log x_{2}+ \\
& z_{13} \cdot e^{B_{\text {Dakin }} \cdot x_{1}} \cdot \log x_{3}+ \\
& z_{23} \cdot \log x_{2} \cdot \log x_{3}+ \\
& z_{123} \cdot e^{B_{\text {Dakin }} \cdot x_{1}} \cdot \log x_{2} \cdot \log x_{3}
\end{aligned}
$$

Table 4: Necessary number of tests for a certain accuracy when each factor has two levels.

The effect of the individual factors and the respective interaction, can be read off at the parameters $z_{i}$. A comparison of these parameters provides information about which effect contributes significantly to the aging of the insulation system and which effects are negligible with respect to service life. When designing robust insulation systems, special attention should be paid to the effects with a high contribution to aging.

\subsection{Miner's rule}

With the mathematical description of the insulation system by multiple regression and the efficient parameterization with the DoE, the lifetime of the insulation system at a certain load can be estimated. The lifetime model will now be extended to estimate the percentage of lifetime consumption due to a variable load spectrum. This is especially useful for applications with strong load fluctuations such as in the automotive industry.

Using the Miner's rule, the lifetime consumption of individual loads of a load spectrum can be calculated. It is assumed that the insulation system is loaded with a constant load for a short period of time. The duration of the constant load $l_{\mathrm{i}}$ is related to the total lifetime of the insulation system at the same load $L i$. 


\begin{tabular}{|c|c|c|c|c|c|c|c|c|c|c|c|c|c|}
\hline Measuring points & 3 & 4 & 5 & 6 & 7 & 8 & 9 & 10 & 11 & 12 & Resolution & Mix & Evaluation \\
\hline 4 & $2^{3-1}$ & & & & & & & & & & 1 & Factor and 2-fold & critical \\
\hline 8 & \begin{tabular}{|c|}
$2^{3}$ \\
complete
\end{tabular} & $2^{4-1}$ & $2^{5-2}$ & $\begin{array}{l}2^{6-3} \\
1\end{array}$ & $\begin{array}{l}2^{7-4} \\
1\end{array}$ & & & & & & "I & $\begin{array}{l}\text { Faktor and } 3 \text {-fold } \\
2 \text {-fold and } 2 \text {-fold }\end{array}$ & critical \\
\hline 16 & & $\begin{array}{c}2^{4} \\
\text { complete }\end{array}$ & $2_{\text {III }}^{5-1}$ & $\begin{array}{c}2^{6-2} \\
11\end{array}$ & $\begin{array}{l}2^{7-3} \\
11\end{array}$ & $\begin{array}{c}2^{8-4} \\
\text { II }\end{array}$ & $\begin{array}{l}2^{9-5} \\
1\end{array}$ & $\begin{array}{c}2^{10-6} \\
1\end{array}$ & $\begin{array}{l}2^{11-7} \\
1\end{array}$ & $\begin{array}{l}2^{12-8} \\
1\end{array}$ & III & $\begin{array}{l}\text { Factor and } 4 \text {-fold } \\
2 \text {-fold and } 3 \text {-fold }\end{array}$ & uncritical \\
\hline 32 & & & $\begin{array}{c}2^{5} \\
\text { complete }\end{array}$ & $\begin{array}{l}2^{6-1} \\
\text { IV }\end{array}$ & $\begin{array}{c}2^{7-2} \\
11\end{array}$ & $\begin{array}{l}2^{8-3} \\
\text { II }\end{array}$ & $\begin{array}{c}2^{9-4} \\
\text { II }\end{array}$ & $\begin{array}{c}2^{10-5} \\
11\end{array}$ & $\begin{array}{l}2^{11-6} \\
\text { II }\end{array}$ & $\begin{array}{l}2^{12-7} \\
\text { II }\end{array}$ & IV & higher resolution & uncritical \\
\hline 64 & & & & $\begin{array}{c}2^{6} \\
\text { complete }\end{array}$ & $\begin{array}{l}2^{7-1} \\
V\end{array}$ & $\begin{array}{c}2^{8-2} \\
\text { III }\end{array}$ & $\begin{array}{c}2^{9-3} \\
11\end{array}$ & $\begin{array}{c}2^{10-4} \\
\text { II }\end{array}$ & $\begin{array}{l}2^{11-5} \\
\text { II }\end{array}$ & $\begin{array}{c}2^{12-6} \\
\text { II }\end{array}$ & v & higher resolution & uncritical \\
\hline 128 & & & & & $\begin{array}{c}2^{7} \\
\text { complete }\end{array}$ & $\begin{array}{l}2^{8-1} \\
\text { VI }\end{array}$ & $\begin{array}{l}2^{9-2} \\
\text { IV }\end{array}$ & $\begin{array}{c}2^{10-3} \\
\text { III }\end{array}$ & $\begin{array}{l}2^{11-4} \\
\text { III }\end{array}$ & $\begin{array}{l}2^{12-5} \\
\text { II }\end{array}$ & $\mathrm{VI}$ & higher resolution & uncritical \\
\hline
\end{tabular}

Table 3: Overview and evaluation of fractional factorial designs for the lifetime of the insulation system [8].

The sum of the individual loads result in the percentage lifetime consumption. The closer the sum of the individual loads approaches the value 1 , the more lifetime is consumed and the more likely the insulation system is to fail. Miner's rule is given in equation (16), where $L \%$ is the percentage lifetime consumption

$$
\sum_{i=1}^{n} \frac{l_{i}}{L_{i}}=L_{\%}
$$

\section{Conclusion}

In this paper a methodology for the lifetime calculation of the insulation system of electrical machines is presented. Besides the prediction of the lifetime, the effects of individual factors and the interactions considered in the modelling can be evaluated and compared. The model can be used in the design process of electrical machines or can be used as a virtual test bench, where the lifetime can be investigated at different loads. Figure 5 shows an overview of the entire methodology. The WLTP is shown as an example of the load spectrum, since the model can be used particularly well in the automotive industry.

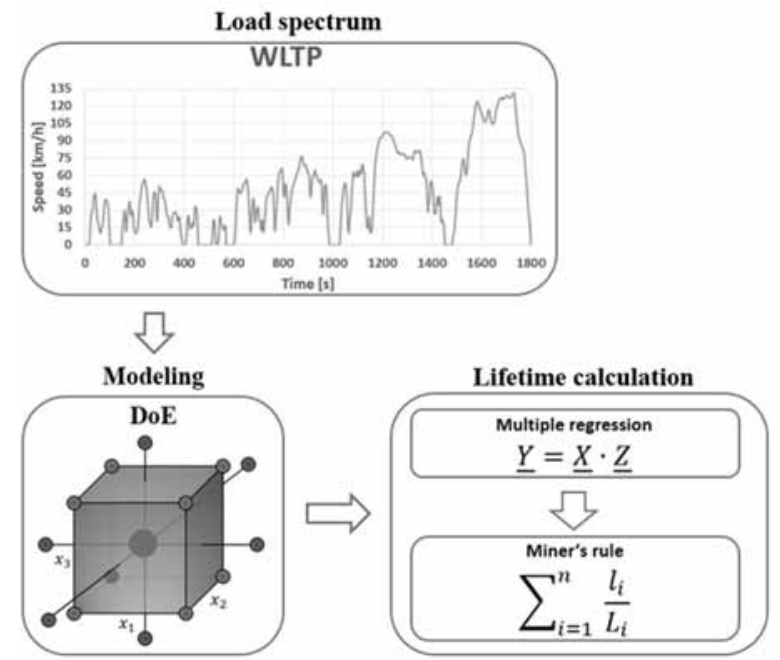

Figure 5: Overview of the modelling methodology.
Currently, durability tests are being carried out to apply the model to a real machine. Since the focus of this article is on modelling, the results and aging effects will be published in a separate article.

\section{References}

[1] Bonnett A H, Yung C. Increased Efficiency Versus Increased Reliability. Iin IEEE Industry Applications Magazine, vol. 14, no. 1, pp. 29-36, Jan.-Feb. 2008, doi: 10.1109/MIA.2007.909802

[2] Brütsch R, Tari M, Fröhlich K, Weiers T, Vogelsang R. Insulation Failure Mechanisms of Power Generators [Feature Article]. IEEE Electrical Insulation Magazine, vol. 24, no. 4, pp. 17-25, July-Aug. 2008. doi: 10.1109/MEI.2008.4581636

[3] Gjerde A C. Multifactor ageing models - origin and similarities. IEEE Electrical Insulation Magazine, vol. 13, no. 1, pp. 6-13, Jan.-Feb. 1997. doi: $10.1109 / 57.567392$.

[4] Artbauer J. Electric Strength of Polymers. Journal of Physics D, Appl. Phys., Vol. 29, pp. 446-56, 1996.

[5] Parpal J L, Crine J P, Chinh Dang. Electrical aging of extruded dielectric cables. A physical model. IEEE Transactions on Dielectrics and Electrical Insulation, vol. 4, no. 2, pp. 197-209, April 1997. doi: 10.1109/94.595247.

[6] Pietrini G, Barater D, Immovilli F, Cavallini A, Franceschini G. Multi-stress lifetime model of the winding insulation of electrical machines. 2017 IEEE Workshop on Electrical Machines Design, Control and Diagnosis (WEMDCD), Nottingham, 2017, pp. 268-274. doi: 10.1109/WEMDCD.2017.7947758.

[7] Dakin T. W. Electrical Insulation Deterioration Treated as a Chemical Rate Phenomenon. Transactions of the American Institute of Electrical Engineers, vol. 67, no. 1, pp. 113-122, Jan. 1948. doi: 10.1109/T-AIEE.1948.5059649

[8] Kleppmann W. Versuchsplanung Produkte und Prozesse optimieren. 2013 Carl Hanser Verlag München Wien, ISBN, 978-3-446-43752-4. 


\title{
A Tutorial-oriented Approach to ARGESIM Benchmark C11 'SCARA Robot' in MATLAB, Simulink and Stateflow
}

\author{
Johannes Leindecker, Maximilian Zechmeister-Machhart, Felix Gauss, Philipp Wiegard \\ Inst. of Mechanics and Mechatronics \& Inst. of Analysis and Scientific Computing, TU Wien, \\ Wiedner Hauptstrasse 8-10, 1040 Vienna, Austria; johannes.leindecker@tuwien.ac.at
}

SNE 31(2), 2021, 101 - 110, DOI: 10.11128/sne.31.bne11.10569 Received: January 13, 2021; Revised April 25, 2021;

Accepted: May 15, 2021

SNE - Simulation Notes Europe, ARGESIM Publisher Vienna ISSN Print 2305-9974, Online 2306-0271, www.sne-journal.org

Abstract. This Educational Benchmark Note presents a tutorial-oriented approach to 'ARGESIM Benchmark C11 SCARA Robot', an SNE Student Note compiled by master students of Mechanical Engineering. Students have described for students necessary state space models for the SCARA robot, and algorithmic preparations for implementation in MATLAB and Simulink, including proper state limitations. Furthermore, for collision handling of the robot movement, benefits of a state automata-based implementation in Stateflow is given. The simulations compare explicit and implicit model versions and efficiency of different ODE solvers, and present a basic animation.

\section{Model Description}

Mechanical System. The mechanical system, that is investigated in this Benchmark, is a three-axes robot arm, called SCARA (Selective Compliance Assembly Robot Arm). It has two vertical revolute joints and one vertical prismatic joint, with which it can change the position of its mounted tool tip.

One can describe the equations of motion in compact form of an implicit second order system of differential equations:

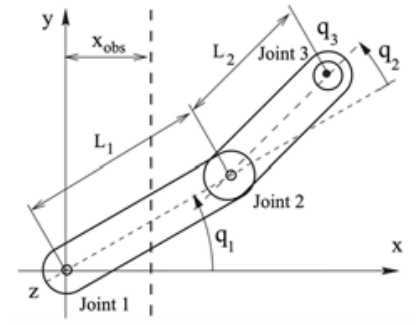

$$
M \ddot{\vec{q}}=\vec{b}
$$

Figure 1: Mechanical structure of the SCARA robot [1]

In this case, vector $\vec{q}$ includes the joint angles $q_{1}$ and $q_{2}$ and the joint distance $q_{3}$. The mass matrix $\boldsymbol{M}$ is a $3 \times 3$ block-diagonal matrix:

$$
\boldsymbol{M}=\left(\begin{array}{ccc}
m a_{11} & m a_{12} & 0 \\
m a_{21} & m a_{22} & 0 \\
0 & 0 & m a_{33}
\end{array}\right)
$$

To calculate the moments of inertia (eq. (8) - (10)), we assume that there is a homogeneous mass distribution of the two rods (mass $m_{1}$ and $m_{2}$ ) along the length $L_{1}$ and $L_{2}$, the mass of the load and the vertical drive motor is known and the moment of inertia of the rotating parts is defined.

$$
\begin{aligned}
& m a_{11}=\Theta_{1}+2 \Theta_{2} \cos \left(q_{2}\right)+\Theta_{3} \\
& m a_{12}=\Theta_{2} \cos \left(q_{2}\right)+\Theta_{3} \\
& m a_{21}=m a_{12} \\
& m a_{22}=\Theta_{3} \\
& m a_{33}=m_{3 L}+\Theta_{3 m o t} u_{3}^{2} \\
& \Theta_{1}=\left(\frac{m_{1}}{3}+m_{2}+m_{3}\right) L_{1}^{2} \\
& \Theta_{2}=\left(\frac{m_{2}}{2}+m_{3}\right) L_{1} L_{2} \\
& \Theta_{3}=\left(\frac{m_{2}}{3}+m_{3}\right) L_{2}^{2} \\
& m_{3}=m_{3 A}+m_{3 L}
\end{aligned}
$$

Vector $\vec{b}$, which describes the right-hand side of the differential equation, is a function of the joint torques $T_{1}(t)$ and $T_{2}(t)$ and the joint force $T_{3}(t)$.

$$
\begin{aligned}
& b_{1}=T_{1}+\Theta_{2}\left(2 \dot{q}_{1} \dot{q}_{2}+\dot{q}_{2}^{2}\right) \sin \left(q_{2}\right) \\
& b_{2}=T_{2}-\Theta_{2} \dot{q}_{1}^{2} \sin \left(q_{2}\right) \\
& b_{3}=T_{3}-m_{3 L} g
\end{aligned}
$$

Servo Motor and PD-Control. Due to the usage of a PD-control (Proportional-Derivative), which controls position errors and joint velocities, there is a limitation for the mechanical system that has to be observed. This limitation is the armature voltage (eq. (15)), restricted by $\pm U_{\text {ireg }}^{\max }$ (eq. (16)) during the regular operation of the SCARA. The armature voltage is a function of the proportional gains $P_{i}$, the derivative gains $D_{i}$ and the target and current value of the joints:

$$
U_{i}=P_{i}\left(\hat{q}_{i}-q_{i}\right)-D_{i} \dot{q}_{i}, \quad i=1,2,3
$$




$$
U_{a i}=\left[-U_{i}^{\max } \leq U_{i} \leq U_{i}^{\max }\right], \quad i=1,2,3
$$

In case of an emergency, the armature voltage has a bigger restriction zone $\left( \pm U_{\text {imax }}^{\max }\right)$ and therefore allows a higher armature voltage. This emergency feature is used in the collision avoidance later on.

Another limitation of the mechanical system is the armature current, due to the engineering property of a servo motor. Using a first order differential equation (eq. (17)), the electrical relationship of the armature is expressible with the servo motor constant $k_{T i}$, gear ratio $u_{i}$, resistance $R_{i}$ and inductance $L_{i}$ :

$$
\begin{aligned}
& \dot{I}_{i}=\frac{\left(U_{a i}-k_{T i} u_{i} \dot{q}_{i}-R_{a i} I_{a i}\right)}{L_{a i}}, \quad i=1,2,3 \\
& I_{a i}=\left[-I_{i}^{\text {max }} \leq I_{i} \leq I_{i}^{\text {max }}\right], \quad i=1,2,3
\end{aligned}
$$

$I_{i}^{\max }$ (eq. (19)) can be computed with the maximum permitted torque $T_{i}^{\max }$ and the servo motor constant $k_{T i}$ :

$$
I_{i}^{\max }=T_{i}^{\max }\left(\frac{\sqrt{3}}{2} k_{T i}\right)^{-1}, \quad i=1,2,3
$$

The armature current is proportional to the joint torques $\left(T_{1}, T_{2}\right)$ and the joint force $\left(T_{3}\right)$ :

$$
T_{i}=u_{i} \frac{\sqrt{3}}{2} k_{T i} I_{a i}, \quad i=1,2,3
$$

Obstacle definition $\boldsymbol{\&}$ collision avoidance. Creating an obstacle, the tool-tip has to avoid, generates the collision avoidance task. The obstacle is defined as

$$
\begin{aligned}
& h=h_{o b s} \forall x \leq x_{o b s} \\
& h=0 \forall x>x_{o b s}
\end{aligned}
$$

with $h_{o b s}=0.2 m$ and $x_{o b s}=0.25 m$

This SCARA is equipped with a distance measuring sensor, which allows regular movement of the tool tip, if the distance between tool tip and obstacle is above the critical distance $d_{\text {crit }}>0.1 \mathrm{~m}$. In case of an undercut of the critical distance $d_{c r i t}$, the armature voltage can have a maximum of $\pm U_{\text {imax }}^{\max }$ to decelerate faster and prevent a crash.

Additional Range Control. Due to the mechanical structure of the SCARA, we need to implement additional range control for the joint angles and the distance $q_{i}$, to achieve a model that pictures the real-life SCARA even better:

$$
\begin{aligned}
& q_{1} \leq \pi \\
& \left|q_{2}\right| \leq \frac{5 \pi}{6} \\
& 0.4 m \geq q_{3} \geq 0 \mathrm{~m}
\end{aligned}
$$

We only let our SCARA robot move its tool tip in a positive $y$-axis area (eq. (25)). That is why the limitation for the angle $q_{1}$ is defined as $q_{1} \leq 180^{\circ}$. Angle $\left|q_{2}\right|$ has a limit of under $180^{\circ}$, because the second rod is above the first one, which means that the tool-tip would crash into the first rod if $\left|q_{2}\right|$ would reach an angle close to $180^{\circ}$ (eq. (28)). The length of the vertical rod $q_{3}$ also needs to be limited to ensure a realistic simulation (eq. (27)).

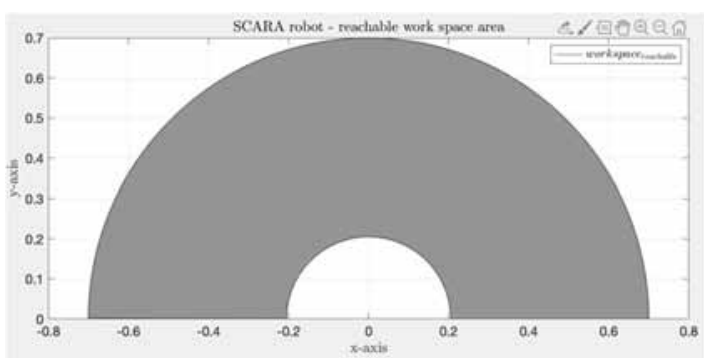

Figure 2: Reachable area of the tool-tip in the $x-y-l e v e l$.

\section{MATLAB Implementation}

Now we create a model of the SCARA movement using MATLAB. At first, we implement the given values and the equations of the definition, such as equation (3) (15), (17), (19) and (20) - (27). These equations provide the needed values to implement the limitation of the current $I_{i}$ from equation (17) and the limitation of the voltage $U_{i}$ from equation (18). The implementation of the mass matrix (eq. (2)) differs, when we compare the explicit with the implicit model. Therefore, the mass matrix implementation gets described in chapter 1.1 and 1.2.

These mentioned implementations are straight forward and didn't cause any problems, but limitating the current $I_{i}$ is not as simple as it may seem. By reason of the inability of the ODE-solver to limit the current itself, we limitated the voltage $U_{i}$ and the derivative of the current $\dot{I}_{i}$. To carry out the limitation for the voltage $U_{i}$, we simply use ,if' loops that constantly compare the current value of the voltage $U_{i}$ with the maximum allowed voltage $\pm U_{\text {ireg }}^{\max }$. In case of an exceeding, the value for $U_{i}$ gets limited to the maximum allowed voltage $\pm U_{i}^{\max }$.

The limitation of the current $\dot{I}_{i}$ is more difficult (shown in code-snippet 1). We constantly have to compare the current value of the current $I_{i}$ with the maximum allowed current $\pm I_{i}^{\max }$. If the current value $I_{i}$ is higher than its maximum $+I_{i}^{\max }$ and the derivative of the current $\dot{I}_{i}$ negative, the derivative of the current $\dot{I}_{i}$ is not limited because the negative inclination of the current $I_{i}$ leads to a decrease of the current $I_{i}$. Consequently, the current $I_{i}$ will drop below its maximum $+I_{i}^{\max }$. 
Vice versa if the current $I_{i}$ is lower than its minimum $-I_{i}^{\max }$ and the derivative of the current $\dot{I}_{i}$ is positive. If the current $I_{i}$ is higher (lower) than its maximum $+I_{i}^{\max }$ $\left(-I_{i}^{\max }\right)$ and the derivative of the current $\dot{I}_{i}$ positive (negative), the value of the derivative of the current $\dot{I}_{i}$ gets limited to zero.

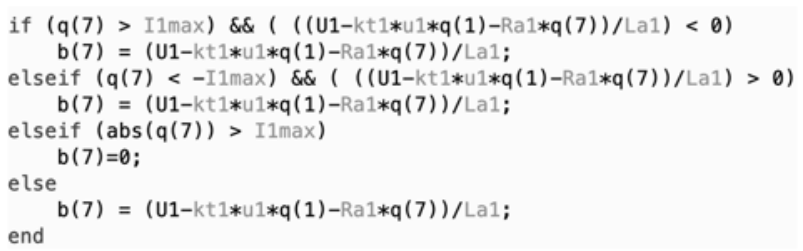

Code-snippet 1: Limitation of the derivative of the current $\dot{I}_{i}$.

There are two possibilities to stop the calculation of the ODE-solver. The computing time can be limited to a fixed value, which is not very accurate when it comes to comparing different solving-times because there might be not enough time to reach the target position or the solver keeps on calculating, even though the SCARA is already very close to the target point or already reached it.

The second possibility uses an implementation of an event-function, that automatically stops the program, as soon as the tool tip reaches the target position. Therefore a so-called event-function "reached_target", which is shown in code-snippet 2 , is used to constantly compare the current position of the the tool tip and the target position. With a difference of $q_{\text {diff }} \leq 0.001 \mathrm{~m}$ between the actual and the target position of the tool tip, the event gets triggered and stops the calculation of the ODE-solver.

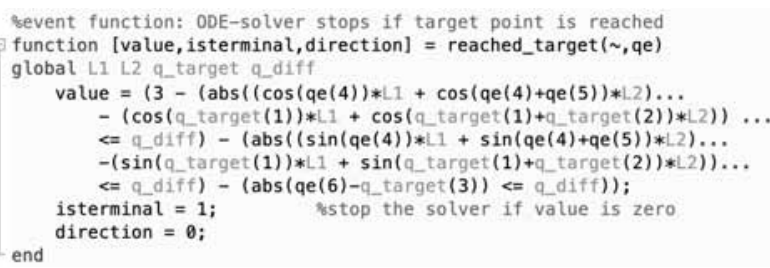

Code-snippet 2: Event that stops the code, as soon as the tool-tip reaches the target position.

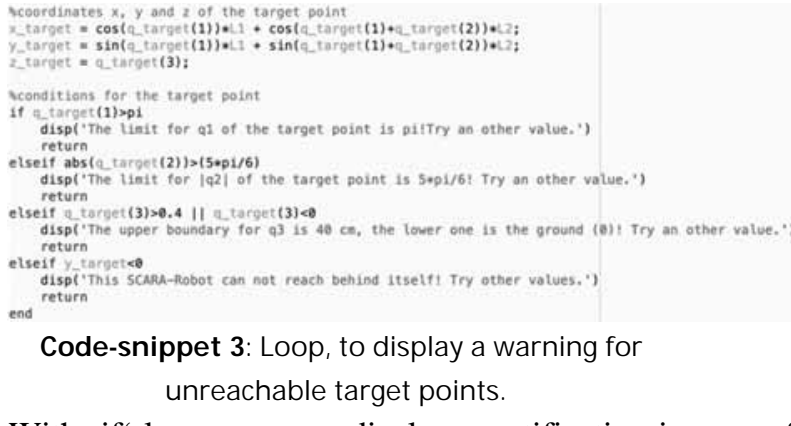

With ,if' loops, we can display a notification in case of an unreachable target position. The notification also gives a short description of the reason for the error, shown in code-snippet 3 .

\subsection{Excplicit Point-to-Point model}

To solve the point-to-Point motion explicitly, we create a 9-row-vector $\dot{\vec{q}}$ that contains the joint velocities $\dot{q}_{i}$, the angles and the distance of the joints $q_{i}$ and the currents of the servo motors $I_{i}$, written as vector $\dot{\vec{q}}=\left[\dot{q}_{i}, q_{i}, I_{i}\right]$. Additionally, the mass matrix $M$ needs to be inverted and multiplied with $\vec{b}$ to receive the joint's angular and directional acceleration $\ddot{\vec{q}}$ :

$$
\ddot{\vec{q}}=\vec{b} M^{-1}
$$

This step is necessary because the ODE-solver in the explicit model can't work with a mass matrix, provided by the odeset() command. Extending vector $\vec{b}$ with $\dot{q}_{i}$ and the limited values of $\dot{I}_{i}$, we also get a 9-row-vector, which basically is the derived $\vec{q}$ vector.

In Figure 3 the changes of the angles and the heigth (q1, q2, q3) over time of the explicit point-to-point model are pictured. So, it can be observed that q1 and q2 have the value 2 and $\mathrm{q} 3$ the value 0.3 when the event-function stops the calculation. Furthermore, figure 4 visualises the movement of the tool tip in the $x-y-z-s y s t e m$.

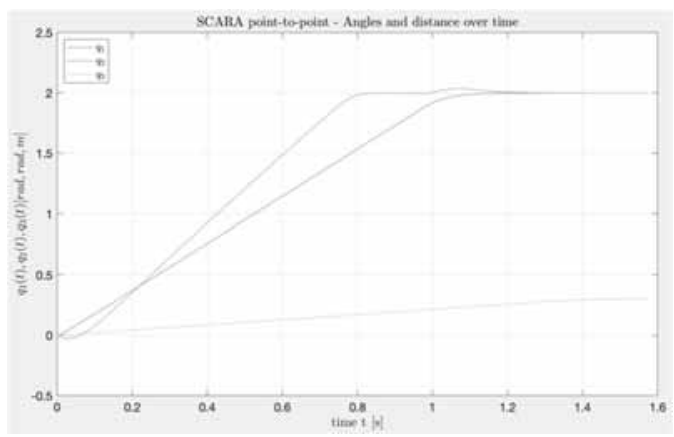

Figure 3: Plot of $q_{1}(t), q_{2}(t)$ and $q_{3}(t)$, depending on $\mathrm{t}$ (explicit point-to-point model, target point $q_{\text {target }}=\left[\begin{array}{lll}2 & 2 & 0.3\end{array}\right]$, ode45).

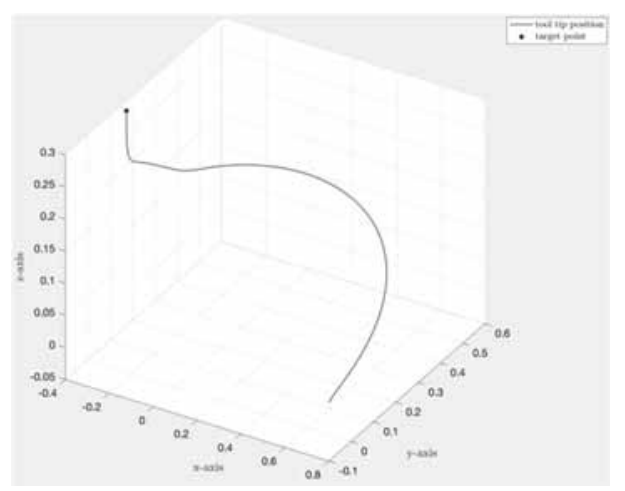

Figure 4: Plot of the movement of the tool tip (explicit point-to-point model, target point $q_{\text {target }}=\left[\begin{array}{lll}2 & 2 & 0.3\end{array}\right]$, ode45). 


\subsection{Implicit Point-to-Point model}

Solving the point-to-point movement implicitly, we need to extend the original $3 \times 3$ mass matrix into a $9 \times 9$ mass matrix (eq. (29)), so the dimensions fit the right side of equation (1).

$$
M=\left(\begin{array}{ccccccccc}
m a_{11} & m a_{12} & 0 & 0 & 0 & 0 & 0 & 0 & 0 \\
m a_{21} & m a_{22} & 0 & 0 & 0 & 0 & 0 & 0 & 0 \\
0 & 0 & m a_{33} & 0 & 0 & 0 & 0 & 0 & 0 \\
0 & 0 & 0 & 1 & 0 & 0 & 0 & 0 & 0 \\
0 & 0 & 0 & 0 & 1 & 0 & 0 & 0 & 0 \\
0 & 0 & 0 & 0 & 0 & 1 & 0 & 0 & 0 \\
0 & 0 & 0 & 0 & 0 & 0 & 1 & 0 & 0 \\
0 & 0 & 0 & 0 & 0 & 0 & 0 & 1 & 0 \\
0 & 0 & 0 & 0 & 0 & 0 & 0 & 0 & 1
\end{array}\right)
$$

The ODE-solver in the implicit model can work with a mass matrix, provided by the odeset() command, which means that we don't have to invert our mass matrix $M$ anymore. Hence, we provide the ODE-solver the maximum step size, the 9x9 mass matrix and the event for reaching the target by the odeset () command. Limiting the current $I_{i}$ and stopping the program happens in the same way as in the explicit model. The numerical accuracy is the same as in the explicit model. Hence, the graphical visualitaion is equal to Figure 3 and Figure 4 and not shown for the implicit model again.

\subsection{Results of the Point-to-Point movement}

We compare the ODE solvers ode45, ode23, ode23t, ode $23 \mathrm{~s}$, ode $23 \mathrm{tb}$, ode113, and ode15s for the point-topoint movement, target point $q_{\text {target }}=\left[\begin{array}{lll}2 & 2 & 0.3\end{array}\right]$ :

\begin{tabular}{ccc} 
ODE-solver & $\begin{array}{c}\text { explicit solving- } \\
\text { time }\end{array}$ & $\begin{array}{c}\text { implicit solving- } \\
\text { time }\end{array}$ \\
\hline ode45 & $0.756971 \mathrm{~s}$ & $0.826150 \mathrm{~s}$ \\
\hline ode23 & $0.572606 \mathrm{~s}$ & $0.838872 \mathrm{~s}$ \\
\hline ode23t & $0.270100 \mathrm{~s}$ & $0.485513 \mathrm{~s}$ \\
\hline ode23s & $3.851616 \mathrm{~s}$ & - \\
\hline ode23tb & $0.299691 \mathrm{~s}$ & $0.595774 \mathrm{~s}$ \\
\hline ode113 & $0.878708 \mathrm{~s}$ & $1.069086 \mathrm{~s}$ \\
\hline ode15s & $0.244320 \mathrm{~s}$ & $0.358710 \mathrm{~s}$
\end{tabular}

Table 1: Explicit and implicit solving-times of different ODE-solvers for the point-to-point movement with target point $q_{\text {target }}=\left[\begin{array}{lll}2 & 2 & 0.3\end{array}\right]$.

The measured explicit system is always faster than the implicit one. Another factor for the solving-time is the step size control of the solver itself. Through a high step size control, like in the ode23t, ode23tb and ode15s, the solving-time is very short. Thereupon, a solver like the ode45, which has a low step size control, has a longer solving-time. In addition, a correlation between the step size control and the accuracy of a solver exists. For example, ode23t, ode23tb and ode15s are fast but also have a low accuracy. Besides, the ode 45 has a medium accuracy, due to its low step size control.

\subsection{Explicit collision avoidance model}

The explicit collision avoidance works in the same way as the explicit point-to-point movement, using the inverted mass matrix $M$. But to implement the collision avoidance, we extend the explicit point-to-point model with various cases which are automatically selected, regarding the dimensions of the obstacle and the target position.

An ,if ' loop, which is partly shown in code-snippet 4, chooses the right sequence of event functions. In this code snippet 4 case no. 1, 2 and the beginning of case no. 3 are pictured. The selected case depends on where the target point is located. If an event function detects a zero transition it stops the current ODE-solver and another one, with a new dynamical structure, has to be started using the last entries of the stopped ODE-solver as starting values for the new one. All times and values of the different used ODEs are attached in the vector te and vector qe to get a gapless simulation.

We differ now from case no. 1 to case no. 5. The differences are described in the following.

Case no. 1. In case no.1 (Figure 5), the target position is located behind the obstacle and above the obstacle's height. It starts the regular point-to-point solver until the tool-tip reaches the critical distance $d_{\text {crit }}=0.1 \mathrm{~m}$. After the event of the critical distance between the obstacle and the tool tip occurs, a new solver is called which only allows movement of $q_{3}$ (shown in code-snippet 5). This means that the SCARA drives vertically upwards, until the tool-tip is higher than the height of the obstacle.

Then, the standard point-to-point solver is called again to reach the target position.

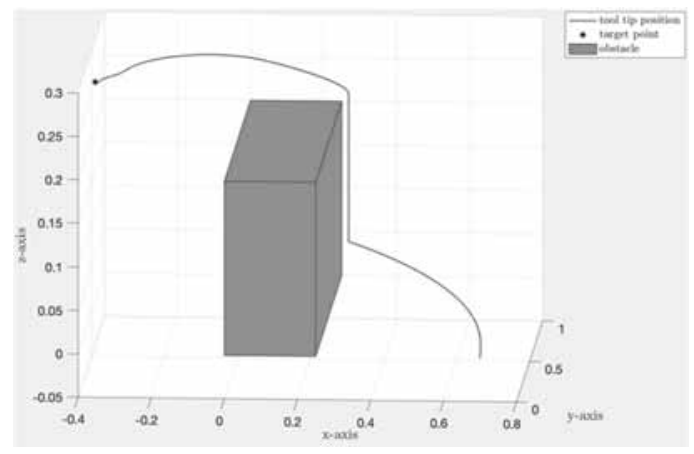

Figure 5: Plot of case no. 1 (explicit collision avoidance model, target point $q_{\text {target }}=\left[\begin{array}{lll}2 & 2 & 0.3\end{array}\right]$, ode45). 


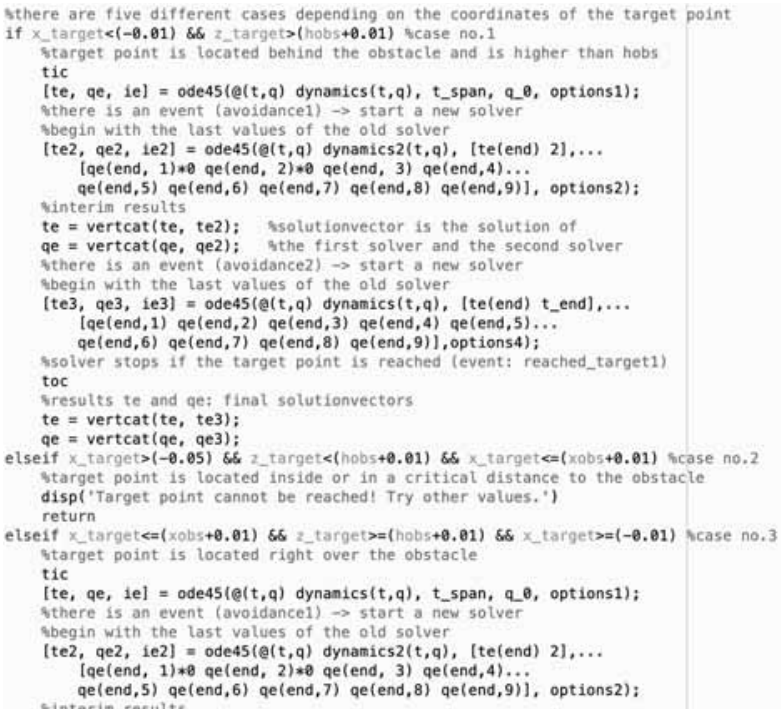

Code-snippet 4: Case no.1, 2 and partly 3, chosen depending on the position of the target point.

first 3 equations have to be multiplied by the inverted mass matrix

Code-snippet 5: Limiting the movement of the SCARA robot to only joint 3.

Case no. 2. In case no. 2, the target position lies within the obstacle or its critical surrounding. A notification of the error gets displayed.

Case no. 3. In case no. 3, the target position is located straightly above the obstacle's height. The utilized procedure is the same as in case no. 1.

Case no. 4. In case no. 4 (fig. 6-8), the target position is located behind the obstacle and below the obstacle's height. Here, the solver for the point-to-point movement is used until the collision warning event gets triggered, followed by the vertical upward movement of the tool-tip as in case no. 1. As soon as the tool tip is higher than the obstacles height, another solver is used, which only allows movement in joint 1 and 2 . This leads to a movement of the SCARA parallel to the obstacles top side. After the tool tip passes the obstacle, the solver for the point-to-point movement is used again to finally reach the target point.

Case no. 5. In case no. 5, the target position is located in front of the obstacle. No collision avoidance occurs, which makes this case to a simple point-to-point movement; events are used to stop the program when the tool tip reaches the target position.

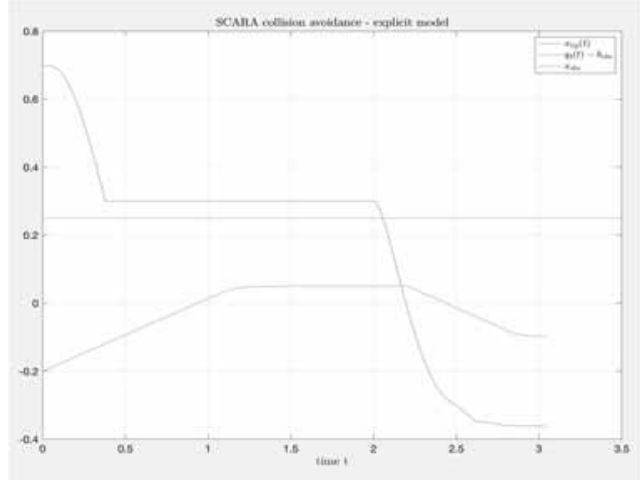

Figure 6: Plot of $x_{t i p}(t), q_{3}(t)-h_{o b s}$ and $x_{o b s}$ over time $t$ (case no. 4, explicit collision avoidance model, target point $q_{\text {target }}=\left[\begin{array}{lll}2 & 2 & 0.1\end{array}\right]$, ode45).

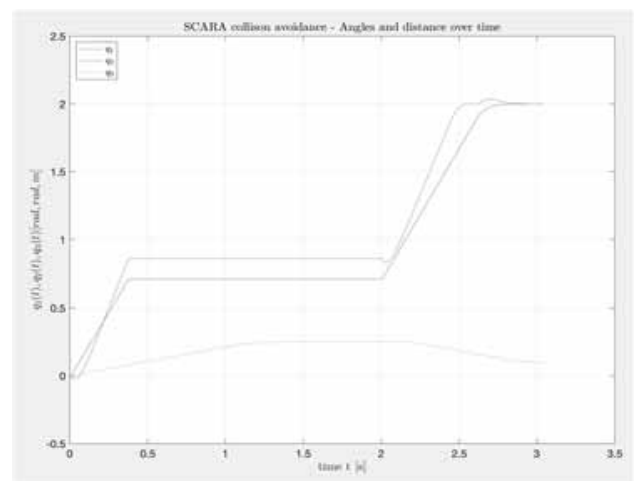

Figure 7: Plot of $q_{1}(t), q_{2}(t)$ and $q_{3}(t)$, depending on $t$ (case no. 4, explicit collision avoidance model, target point $q_{\text {target }}=\left[\begin{array}{lll}2 & 2 & 0.1\end{array}\right]$, ode45).

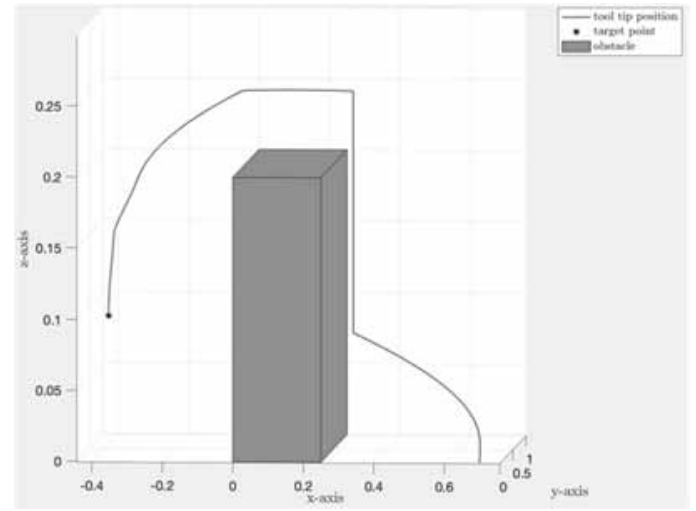

Figure 8: Plot of the case no. 4 (explicit collision avoidance model, target point $q_{\text {target }}=\left[\begin{array}{lll}2 & 2 & 0.1\end{array}\right]$, ode45) .

\subsection{Implicit collision avoidance model}

The implicit collision avoidance model uses the same structure as the explicit collision avoidance model, apart from the handling of the mass matrix. Here, the handling of the mass matrix is the same as in 1.2 Implicit Point-toPoint model. The solutions of the implicit model in case no. 1 are plotted in Figure 9 and Figure 10.

SNE 31(2) - 6/2021 


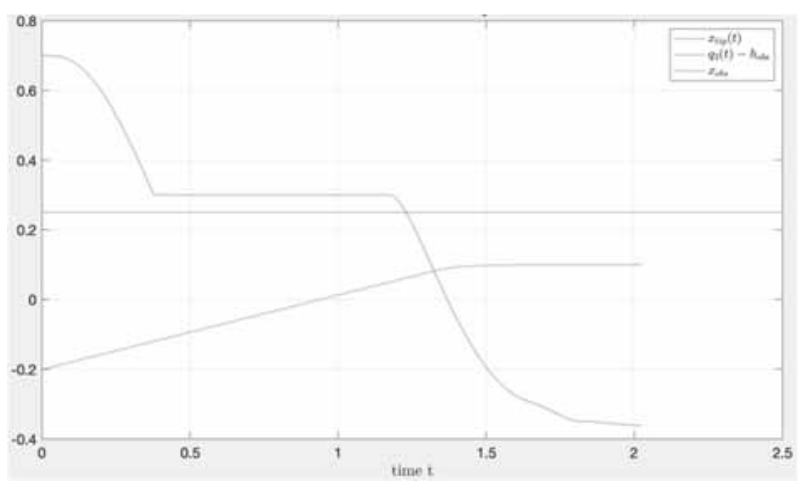

Figure 9: Plot of $x_{t i p}(t), q_{3}(t)-h_{o b s}$ and $x_{o b s}$ over time $t$ (case no. 1, implicit collision avoidance model, target point $q_{\text {target }}=\left[\begin{array}{lll}2 & 2 & 0.3\end{array}\right]$, ode45).

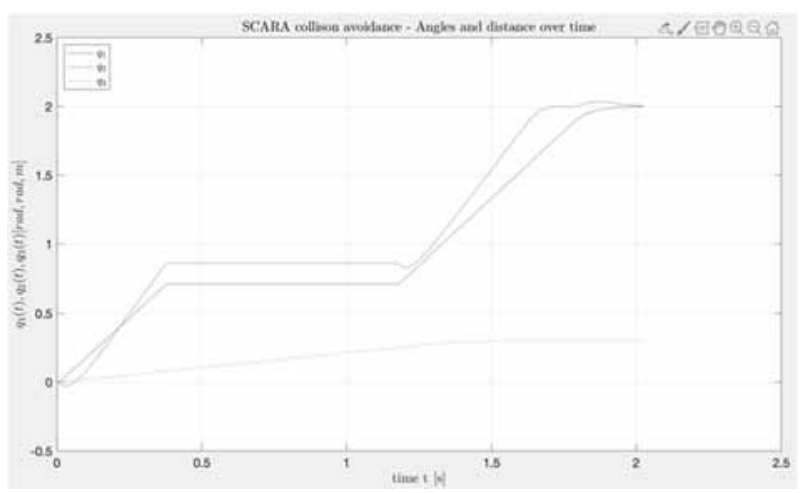

Figure 10: Plot of $q_{1}(t), q_{2}(t)$ and $q_{3}(t)$, depending on $\mathrm{t}$ (case no. 1, implicit collision avoidance model, target point $q_{\text {target }}=\left[\begin{array}{lll}2 & 2 & 0.3\end{array}\right]$, ode45).

\subsection{Results collision avoidance movement}

We compare the following ODE-solvers for the collision avoidance movement with the target point $q_{\text {target }}=$ [2 2 l 0.3 ]: ode45, ode23, ode23t, ode23s, ode23tb, ode113 and ode15s.

\section{ODE-solver explicit solving- implicit solving-} time time

\begin{tabular}{clc}
\hline ode45 & $1.163843 \mathrm{~s}$ & $1.316385 \mathrm{~s}$ \\
\hline ode23 & $0.820086 \mathrm{~s}$ & $0.988098 \mathrm{~s}$ \\
\hline ode23t & $0.458257 \mathrm{~s}$ & $0.581426 \mathrm{~s}$ \\
\hline ode23s & $3.177899 \mathrm{~s}$ & - \\
\hline ode23tb & $0.427696 \mathrm{~s}$ & $0.621490 \mathrm{~s}$ \\
\hline ode113 & $1.240606 \mathrm{~s}$ & $1.259776 \mathrm{~s}$ \\
\hline ode15s & $0.422060 \mathrm{~s}$ & $0.422124 \mathrm{~s}$
\end{tabular}

Table 2: Explicit and implicit solving-times of different ODE-solvers for the collision avoidance movement with target point $q_{\text {target }}=\left[\begin{array}{lll}2 & 2 & 0.3\end{array}\right]$.

\section{Simulink Implementation}

In Simulink, a model for simulating the Point-to-Point movement in an explicit representation and two different models for simulating the Point-to-Point movement with collision avoidance also in an explicit representation were developed. On the one hand a collision avoidance model with the usual Simulink-blocks and a 'Switch'block to switch between the Point-to-Point movement and the collision avoidance movement and on the other hand a Simulink solution with integrated 'Stateflow'states, in which it is also possible to switch between these movements.

All constant parameters necessary for the simulation were given into Simulink over the MATLAB environment. The simulation results were transferred back into MATLAB through 'To Workspace'-blocks for plotting purposes. To solve the explicit Simulink models, the variable step size, one step solver ode45 was used, whereby the relative tolerance was reduced to $1 \mathrm{e}-6$. Since a double integration is possible in Simulink by implementing two 'Integrator'-blocks connected directly one after the other, it is not necessary to transform the descriptive second order differential equations [2]. The resulting $3 \times 3$ mass matrix was implemented in the Simulink and Simulink / Stateflow models using a 'MATLAB Function'-block and then inverted using a 'Product'-block in inversion mode.

As in the MATLAB implementation in order to prematurely end the integration at the target point, a Simulation Abort Condition-subsystem was integrated in all models (see Figure 11). When a precession of 0.001 is reached, the simulation stops by means of a 'Stop'-block.

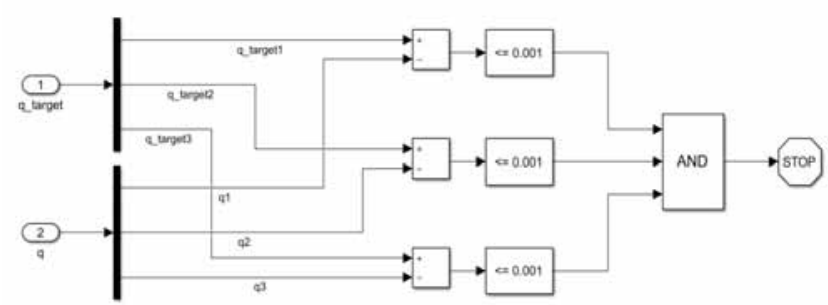

Figure 11: Simulation Abort Condition-subsystem with a logical combination of the differences between the components of the target point location vector $q_{\text {target }}$ and the robot arm location vector $q$, with the 'Stop'-block being activated when a precession of 0.001 is reached.

\subsection{Explicit Point-to-Point model in Simulink}

Figure 12 shows the overall Point-to-Point Simulink model with built-in Control Model-subsystem (shown in Figure 13) and a 'MATLAB Function'-block called dynamics (which contains the equations 12 to 14 ). 
The Control Model-subsystem includes a PD controlsubsystem, which defines the relationship between the distance to the target point and the necessary voltage (see equation 15), as well as a servo motor-subsystem (shown in figure 14), which contains the first order differential equation for determining the current (see equation 17). The resulting current is limited via a limited output of the 'Integrator'-block. The current limit values are calculated using equation 19. Finally, the associated torque is determined in a 'MATLAB Function'-block. The torque is required to calculate the right-hand side of the system of differential equations (see equations 12 to 14 ).

The voltage is limited via a 'Saturation'-block, which is included in the PD control-subsystem. The voltage limit values, like the current limit values, are given constant parameters. The voltage and current limitation are much easier to implement in Simulink than in MATLAB.

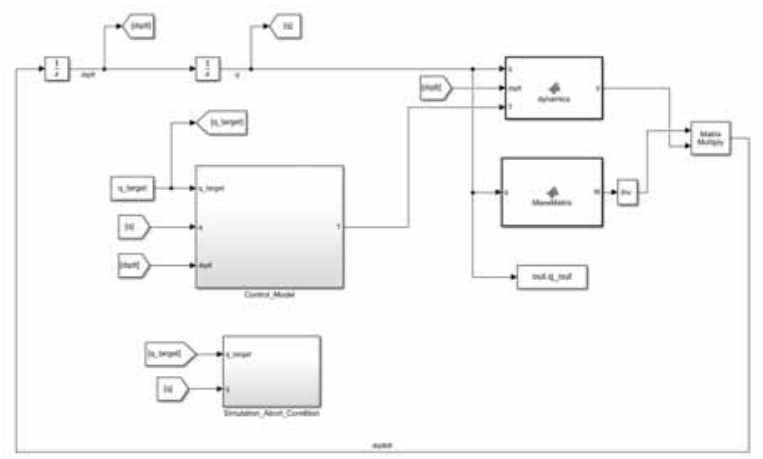

Figure 12: Explicit Point-to-Point Simulink model.
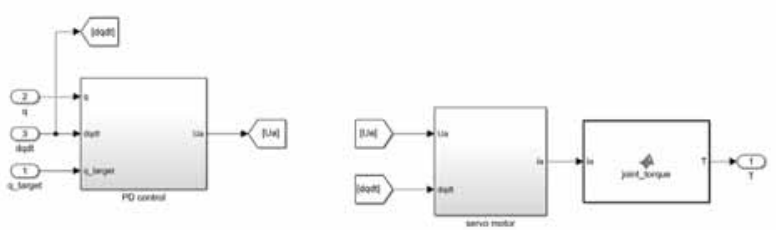

Figure 13: Control Model-subsystem.

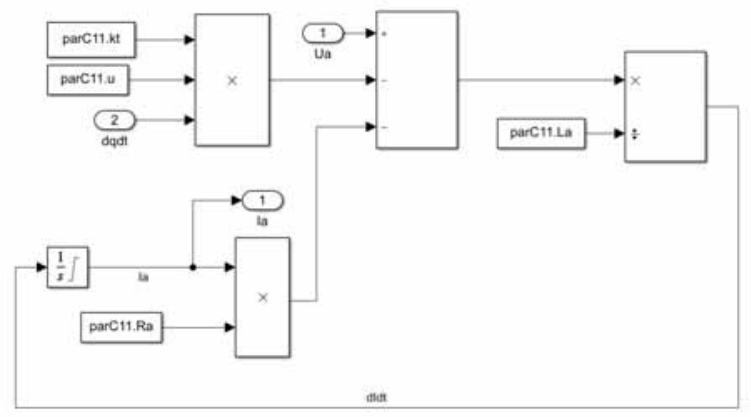

Figure 14: Servo motor-subsystem including an Integrator'-block with limited output.

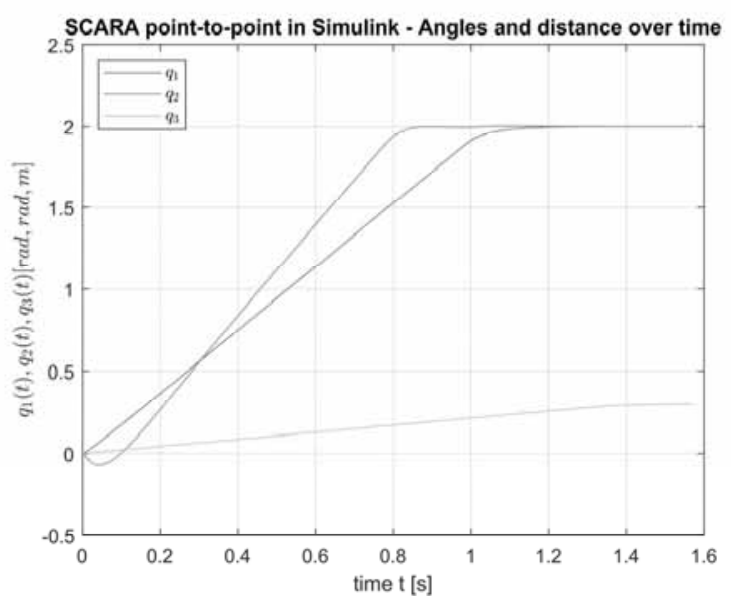

Figure 15: Point-to-Point simulation in Simulink time course of $q_{1}, q_{2}$ and $q_{3}$.

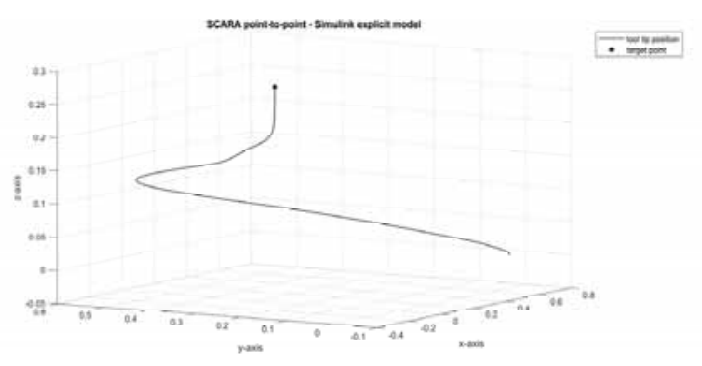

Figure 16: Point-to-Point simulation in Simulink tool-tip path in three-dimensional space.

Results. Figure 15 shows the time course of the two angles $q_{1}$ and $q_{2}$ as well as the distance $q_{3}$ during the Pointto-Point movement (simulated in Simulink). Figure 16 shows the result of the Point-to-Point simulation in Simulink in three-dimensional space with initial value $q=$ $[0 ; 0 ; 0]$ and target point $\hat{q}=[2 ; 2 ; 0.3]$. The Simulink Point-to-Point simulation runtime was 0.143362 seconds.

\subsection{Collision avoidance model in Simulink and Simulink / Stateflow}

Figure 17 shows the overall Point-to-Point with collision avoidance Simulink model with built-in Extended Control Model-subsystem. In order to avoid a collision with a defined object, an additional subsystem was included into the Control Model-subsystem (see Figure 18).

In this additional subsystem two 'Switch'-blocks for switching between Point-to-Point movement and obstacle avoidance are implemented.

If the condition $\left(x_{\text {tip }}-x_{\text {obs }}\right) \leq d_{\text {crit }} \wedge q_{3}<h_{o b s}$ is fulfilled, $\dot{q}$ is changed in such a way that $\dot{q}_{1, o b s}=$ $0.00005 \cdot \dot{q}_{1}, \quad \dot{q}_{2, o b s}=0.00005 \cdot \dot{q}_{2}$ and $\dot{q}_{3, o b s}=\dot{q}_{3}$. $\dot{q}_{\text {obs }}$ is then passed on to the integrator and the dynamicsblock (see Figure 17). 


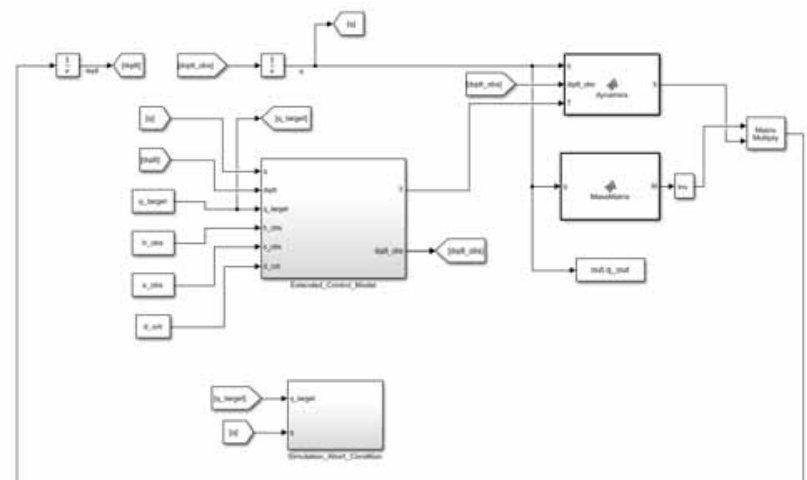

Figure 17: Explicit Point-to-Point with collision avoidance Simulink model.

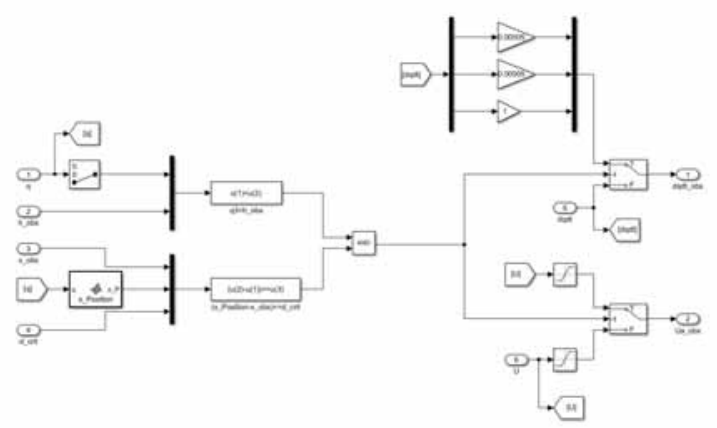

Figure 18: Additional subsystem with two 'Switch'-blocks for switching between different robot arm speeds $\dot{q}$ and maximum voltages.

In the second 'Switch'-block, which is controlled via the same condition, the permissible voltage limits are specified. In the case of obstacle avoidance, the normal maximum voltages (see chapter 2.1 ) are changed to the emergency maximum voltages.

Figure 19 shows the overall Point-to-Point with collision avoidance Simulink model with built-in 'StateflowChart'-subsystem.

When using the Stateflow environment, the task is to define certain states and to specify conditions for when to switch between these states. Point-to-Point movement and collision avoidance were defined as states (see Figure 20). To define the states, 'Simulink state'-blocks were used, in which Simulink models like the one shown in Figure 12 with different $\dot{q}$ and maximum voltages were installed (see Figure 21 and Figure 22). 'State Writer'-blocks were used to transfer the integrated parameters from one state to another. The condition for a change from Point-to-Point movement to obstacle avoidance has not changed compared to the pure Simulink solution. In the opposite direction, the condition that the tool-tip has exceeded the obstacle height is sufficient. The definition of the conditions for changing between the states is much easier in Simulink / Stateflow than in a pure Simulink solution.

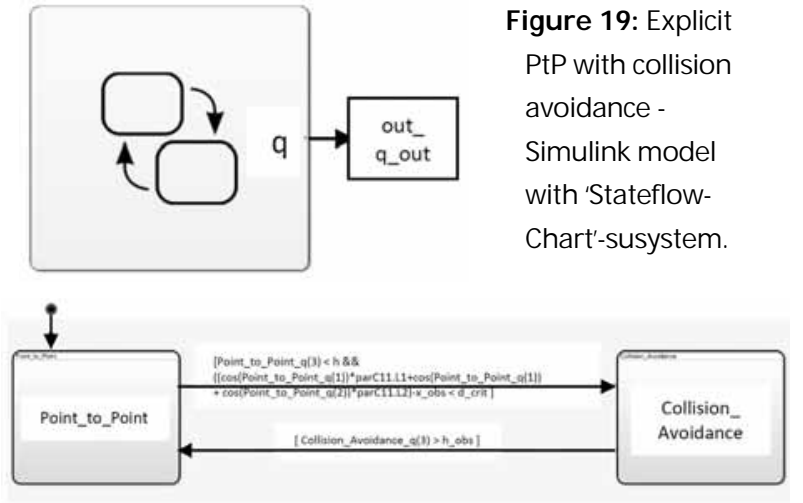

Figure 20: 'Stateflow-Chart'-subsystem with 'Simulink state'-blocks and conditions for a change of state.

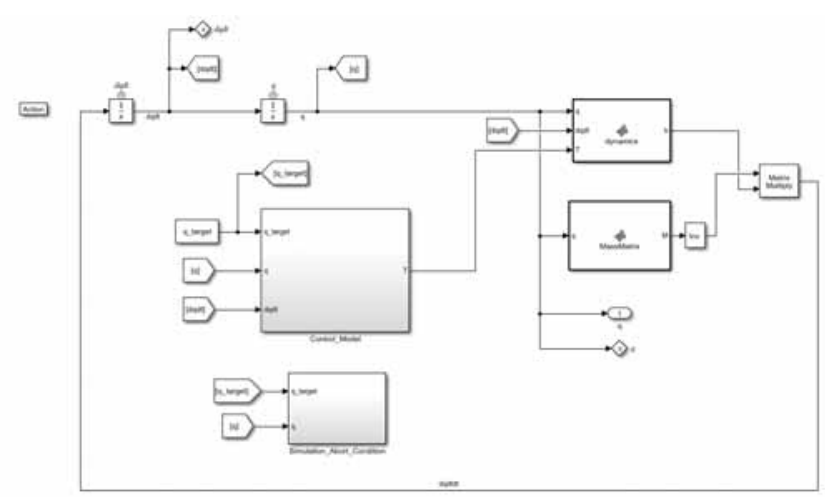

Figure 21: Point-to-Point subsystem.

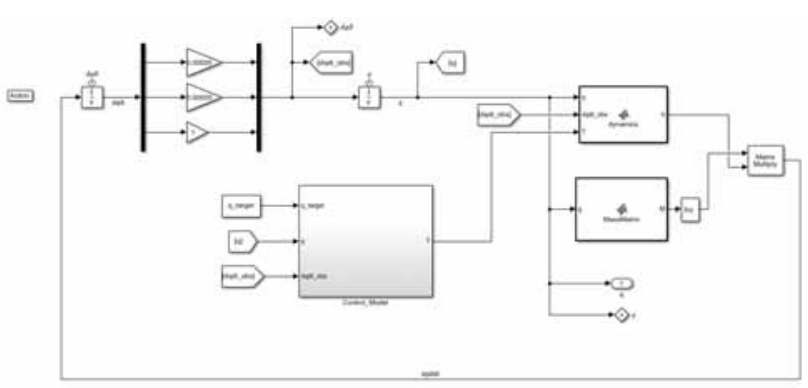

Figure 22: Collision avoidance subsystem.

Results. Figure 23 shows the time course of the two angles $q_{1}$ and $q_{2}$ as well as the distance $q_{3}$ during the Pointto-Point with collision avoidance movement (Simulink).

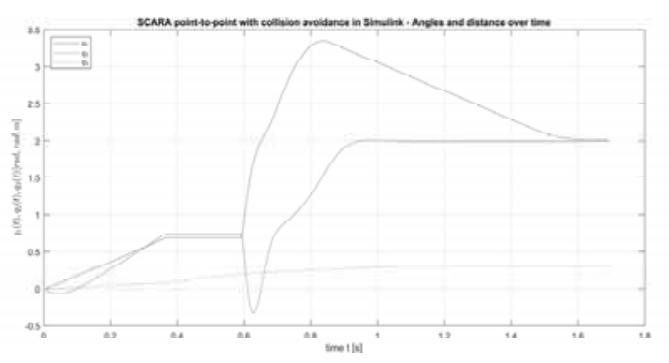

Figure 23: Point-to-Point with collision avoidance simulation in Simulink - time course of $q_{1}, q_{2}$ and $q_{3}$. 
Figure 24 shows the result of the Point-to-Point with collision avoidance simulation in Simulink in three-dimensional space with initial value $q=[0 ; 0 ; 0]$, target point $\hat{q}=[2 ; 2 ; 0.3]$, obstacle height $h_{\text {obs }}=0.2 \mathrm{~m}$, obstacle distance $x_{o b s}=0.25 \mathrm{~m}$ and critical distance $d_{\text {crit }}=0.1 \mathrm{~m}$.

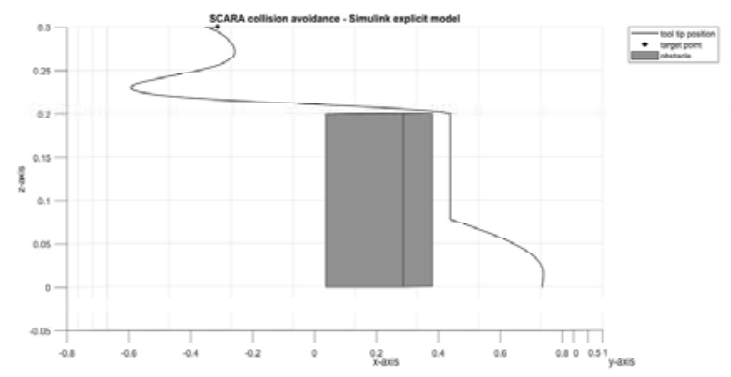

Figure 24: Point-to-Point with collision avoidance simulation in Simulink - tool-tip path in 3D space.

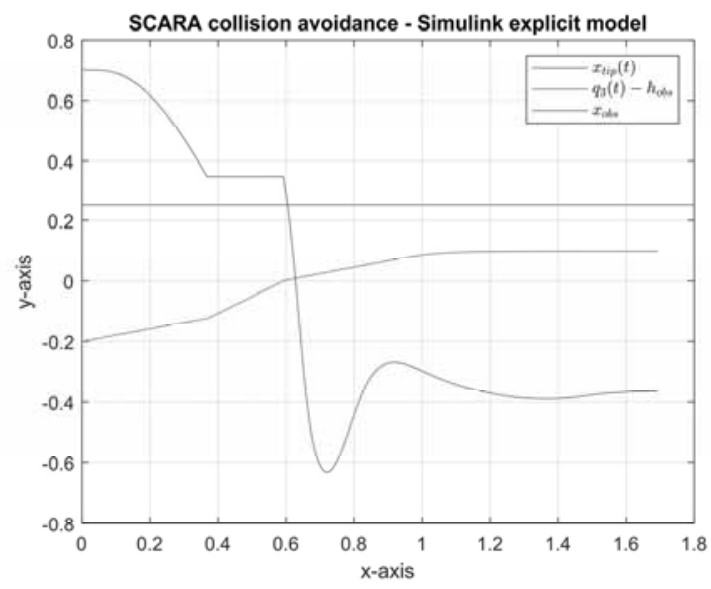

Figure 25: Representation of the Simulink collision avoidance maneuver in two-dimensional space.

The Simulink Point-to-Point with collision avoidance simulation runtime was 0.239948 seconds.

Figure 26 shows the time course of the two angles $q_{1}$ and $q_{2}$ as well as the distance $q_{3}$ during the Point-to-Point with collision avoidance movement (simulated in Simulink / Stateflow).

Figure 27 shows the result of the Point-to-Point with collision avoidance simulation in Simulink / Stateflow in three-dimensional space with initial value $q=[0 ; 0 ; 0]$, target point $\hat{q}=[2 ; 2 ; 0.3]$, obstacle height $h_{o b s}=$ $0.2 \mathrm{~m}$, obstacle distance $x_{o b s}=0.25 \mathrm{~m}$ and critical distance $d_{\text {crit }}=0.1 \mathrm{~m}$.

The Simulink / Stateflow Point-to-Point with collision avoidance simulation runtime was 0.315776 seconds.

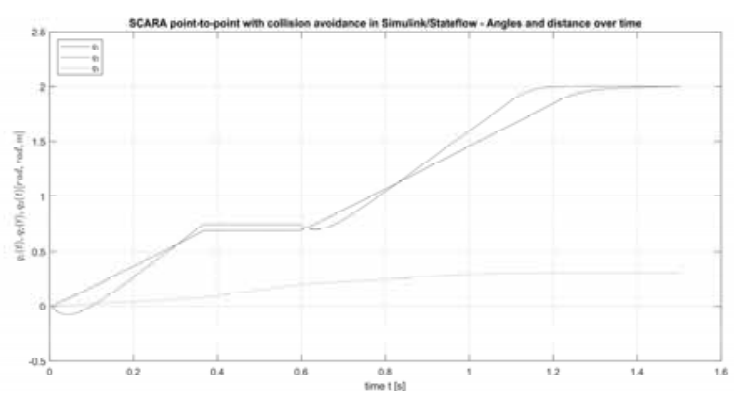

Figure 26: Point-to-Point with collision avoidance simulation in Simulink / Stateflow - time course of $q_{1}, q_{2}$ and $q_{3}$

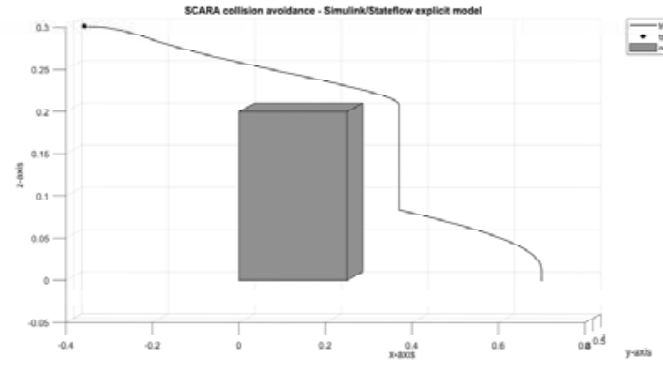

Figure 27: Point-to-Point with collision avoidance simulation in Simulink / Stateflow tool-tip path in three-dimensional space.

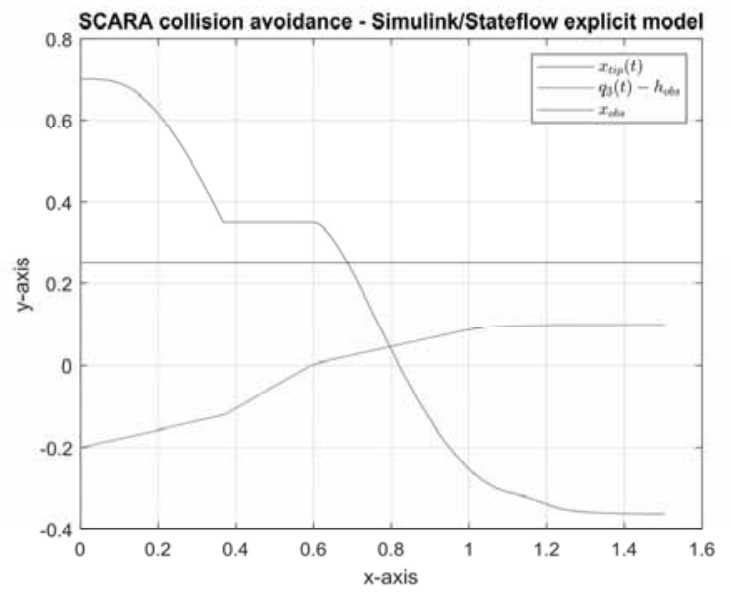

Figure 28: Representation of the Simulink / Stateflow collision avoidance maneuver in 2D space.

\section{Comparison \& Results}

A very essential difference between the MATLAB and the Simulink implementation is the computing time for each model. The direct comparison of the explicit pointto-point models shows, that the Simulink implementation is in our elaboration $\approx 5$ times faster than the MATLAB implementation (both solving times result in the use of the ode45). This observation is confirmed, when we com- 
pare the computing times of the explicit collision avoidance models (both solving times result in the use of the ode45). The Simulink simulation times are significantly shorter than those in MATLAB, since, in contrast to MATLAB, the right side of the differential equation system is mapped using a function and the code is not processed in lines.

On the other hand, using 'MATLAB-function'-blocks in Simulink delays the simulation time.

Another important difference between these two implementation possibilities is the ability of being able to work with a second order differential equation system. MATLAB can't work with a second order differential eqation system, in contrast Simulink can. This makes the Simulink implementation easier because the C11 definition needs no further conversion.

When it comes to the limitation of the voltage and current, the Simulink implementation is a lot easier and faster, compared to the MATLAB implementation. As shown in chapter 2.1, the limitation is done in Simulink with very few steps, while the limitation in MATLAB needs a small expansion, shown in chapter 1 and codesnippet 1 .

\section{Addendum}

Additionally, an animation of the point-to-point movement and the collision avoidance movement is realised in the Matlab code as shown in Figure 29. Only with this animation we got a real feeling how the three rods were moving, and which relative movements are necessary to guid the tool tip of the SCARA to its target point. Furthermore, through the animation we recognized a long waiting time between the vertical and the horizontal movement when the obstacle height was reached so we adapted the setting of the PD control in order to reach the target even faster.

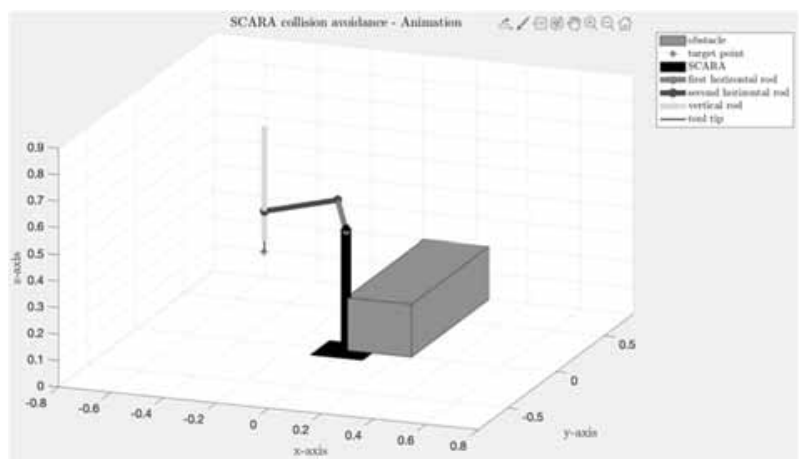

Figure 29: Animation of the collision avoidance movement of the SCARA in a 3D-model.
The animation was implemented with an explicit and with an implicit model, but there was no significant difference. So, further explanations refer to an implicit animation model. For this task the already described code of an implicit collision avoidance was used for this animation. The changes were made in the plot section. First, the SCARA appearance itself had to be modeled. This was realised with different lines for the SCARA body, the rods and the tool tip as shown in the codesnippet 6 .

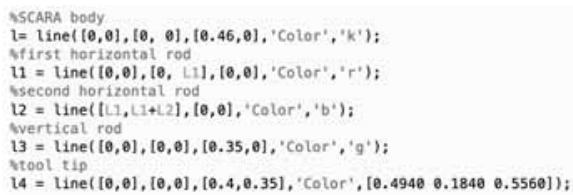

Code-snippet 6: Shaping the appearance of the SCARA with different lines and their spatial position.

Finally, the before calculated values of $q_{i}$ are now used to describe the position of the three rods in code-snippet 7. A ,for' loop is used to plot every calculated position of the rods on their way to the target point with a short pause between the plots to get an animation of the motion of the SCARA.

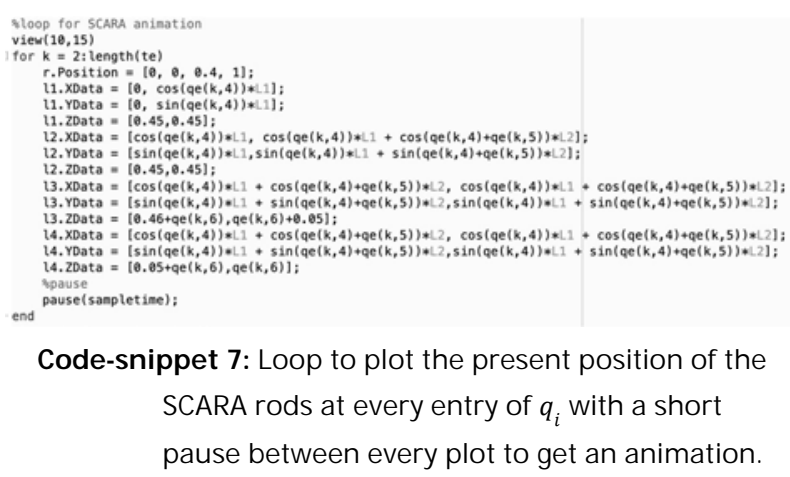

\section{Information}

MATLAB R2019b Update 4 (9.7.0.1296695) and MATLAB R2020a (9.8.0.1323502) was used on macOS Catalina 10.15.7 and Windows 10.0.18363.1139 to develop this elaboration.

\section{References}

[1] Horst Ecker. Comparison 11 (SCARA robot) - Definition. EUROSIM - Simulation News Europe. 1998; 22: 30-32

[2] Mathworks: Products and Services. https://de.mathworks.com (last access on $29^{\text {th }}$ October 2020) 


\section{SNE Simulation News \\ EUROSIM Data and Quick Info}

C DBSS VEIII VES - Virtual EUROSIM Seminar

\begin{tabular}{|c|c|c|}
\hline & SIM S & M Conference 202 \\
\hline & 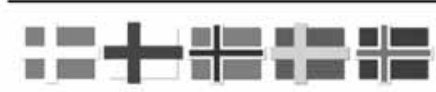 & inland $w$ \\
\hline
\end{tabular}

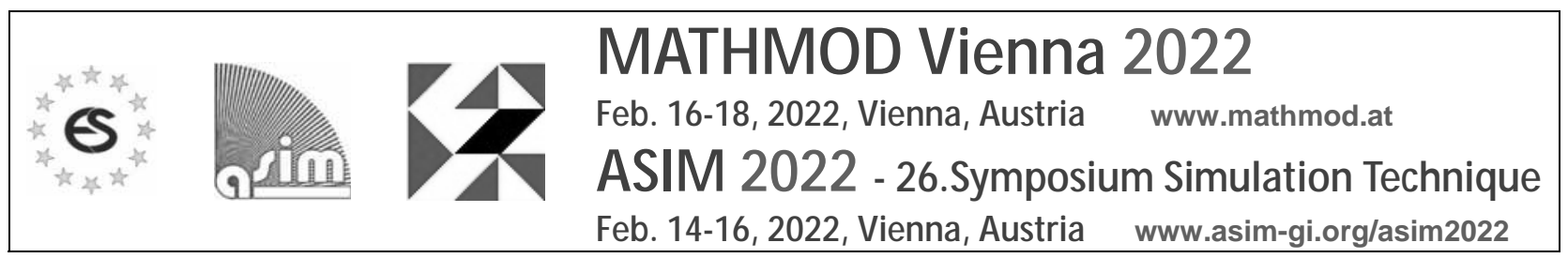

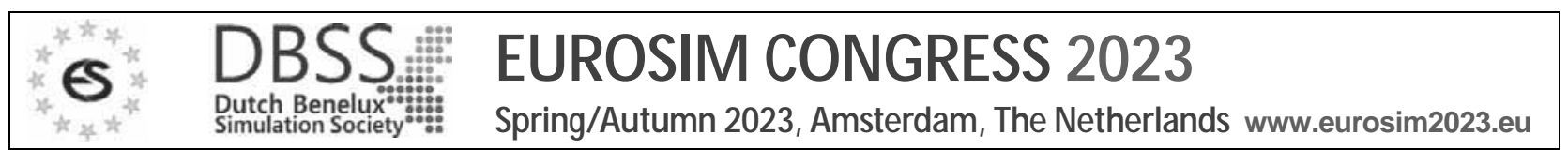

Contents

Short Info EUROSIM N2

Short Info ASIM , CEA-SMSG ........................................ 3

Short Info CSSS, DBSS, LIOPHANT, LSS ......................... 4

Short Info KA-SIM, NSSM, PSCS …............................... 5

Short Info SIMS, SLOSIM, UKSIM ..............................N6

Short Info ROM SIM , Albanian Society .......................N7

Short Info ARGESIM, SNE .........................................

EUROSIM Conferences \& Seminars ............... Back Cover

Simulation Notes Europe SNE is the official membership journal of EUROSIM and distributed / available to members of the EUROSIM Societies as part of the membership benefits.

If you have any information, announcement, etc. you want to see published, please contact a member of the editorial board in your country or the editorial office. For scientific publications, please contact the EiC.

This EUROSIM Data \& Quick Info compiles data from EUROSIM societies and groups: addresses, weblinks, and officers of societies with function and email, to be published regularly in SNE issues. This information is also published at EUROSIM's website www.eurosim.info.

\section{SNE Reports Editorial Board}

EUROSIM Miguel Mujica Mota, m.mujica.mota@hva.nl Nikolas Popper, niki.popper@dwh.at

ASIM A. Körner, andreas.koerner@tuwien.ac.at CEA-SMSG Emilio Jiménez, emilio.jimenez@unirioja.es CSSS Mikuláš Alexík, alexik@frtk.utc.sk DBSS M. Mujica Mota, m.mujica.mota@hva.nl LIOPHANT F. Longo,f.longo@unical.it

LSS Juri Tolujew, Juri.Tolujew@iff.fraunhofer.de KA-SIM Edmond Hajrizi, info@ka-sim.com NSSM Y. Senichenkov, senyb@dcn.icc.spbstu.ru PSCS Zenon Sosnowski, zenon@ii.pb.bialystok.pl SIMS Esko Juuso, esko.juuso@oulu.fi

SLOSIM Vito Logar,vito.logar@fe.uni-lj.si UKSIM David Al-Dabass.david.al-dabass@ntu.ac.uk ROMSIM Constanta Zoe Radulescu, zoe@ici.ro ALBSIM Majlinda Godolja, majlinda.godolja@feut.edu.al

\section{SNE Editorial Office / ARGESIM}

$\rightarrow$ www.sne-journal.org, www.eurosim.info

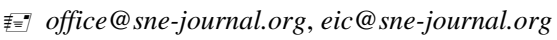

$\triangle$ SNE Editorial Office

Johannes Tanzler (Layout, Organisation)

Irmgard Husinsky (Web, Electronic Publishing)

Felix Breitenecker EiC (Organisation, Authors)

ARGESIM/Math. Modelling \& Simulation Group,

Inst. of Analysis and Scientific Computing, TU Wien

Wiedner Hauptstrasse 8-10, 1040 Vienna, Austria 


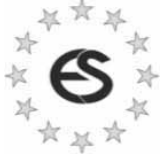

EUROSIM

\section{Federation of European Simulation Societies}

General Information. EUROSIM, the Federation of European Simulation Societies, was set up in 1989. The purpose of EUROSIM is to provide a European forum for simulation societies and groups to promote modelling and simulation in industry, research, and development by publication and conferences. $\rightarrow$ www.eurosim.info

Member Societies. EUROSIM members may be national simulation societies and regional or international societies and groups dealing with modelling and simulation. At present EUROSIM has Full Members and $\mathrm{Ob}$ server Members $(*)$, and Member Candidates (**).

\begin{tabular}{ll}
\hline ASIM & $\begin{array}{l}\text { Arbeitsgemeinschaft Simulation } \\
\text { Austria, Germany, Switzerland }\end{array}$ \\
\hline CEA-SMSG & Spanish M odelling and Simulation Group; Spain \\
\hline CSSS & $\begin{array}{l}\text { Czech and Slovak Simulation Society } \\
\text { Czech Republic, Slovak Republic }\end{array}$ \\
\hline DBSS & $\begin{array}{l}\text { Dutch Benelux Simulation Society } \\
\text { Belgium, Netherlands }\end{array}$ \\
\hline KA-SIM & Kosovo Simulation Society, Kosovo \\
\hline UOPHANT & LOPHANT Simulation Club; Italy \& International \\
\hline LSS & Latvian Simulation Society; Latvia \\
\hline PSCS & Polish Society for Computer Simulation; Poland \\
\hline NSSM & $\begin{array}{l}\text { Russian National Simulation Society } \\
\text { Russian Federation }\end{array}$ \\
\hline SIMS & $\begin{array}{l}\text { Simulation Society of Scandinavia } \\
\text { Denmark, Finland, Norway, Sweden }\end{array}$ \\
\hline SLOSIM & Slovenian Simulation Society; Slovenia \\
\hline UKSIM & $\begin{array}{l}\text { United Kingdom Simulation Society } \\
\text { UK, Ireland }\end{array}$ \\
\hline ALBSIM & Albanian Simulation Society*; Albania \\
\hline ROMSIM & $\begin{array}{l}\text { Romanian Society for M odelling and } \\
\text { Simulation*; Romania }\end{array}$ \\
\hline Societies in Re-Organisation: \\
\hline CROSSIM & Croatian Society f. Simulation M odeling; Croatia \\
\hline FRANCOSIM & Société Francophone de Simulation \\
& Belgium, France \\
\hline HSS & Hungarian Simulation Society; Hungary \\
\hline ISCS & Italian Society for Computer Simulation, Italy \\
\hline
\end{tabular}

EUROSIM Board / Officers. EUROSIM is governed by a board consisting of one representative of each member society, and president, past president, and SNE representative. The President is nominated by the society organising the next EUROSIM Congress. Secretary, and Treasurer are elected out of members of the board.

\begin{tabular}{ll}
\hline President & $\begin{array}{l}\text { M. M ujica M ota (DBSS), } \\
\text { m.mujica.mota@ hva.nl }\end{array}$ \\
\hline Past President & $\begin{array}{l}\text { Emilio Jiménez (CAE-SM SG), } \\
\text { emilio.jimenez@unirioja.es }\end{array}$ \\
\hline Secretary & Niki Popper, niki.popper@ dwh.at \\
\hline Treasurer & $\begin{array}{l}\text { Felix Breitenecker (ASIM) } \\
\text { felix.breitenecker@tuwien.ac.at }\end{array}$ \\
\hline Webmaster & $\begin{array}{l}\text { Irmgard Husinsky, } \\
\text { irmgard.husinsky@tuwien.ac.at }\end{array}$ \\
\hline SNE Editor & F. Breitenecker, eic@ sne-journal.org \\
\hline
\end{tabular}

SNE - Simulation Notes Europe. SNE is EUROSIM's scientific journal with peer reviewed contributions as well as a membership journal for EUROSIM with information from the societies. EUROSIM societies distribute SNE (electronic or printed) to their members as official membership journal. SNE Publishers are EUROSIM, ARGESIM and ASIM.

\begin{tabular}{ll}
\hline SNE & Felix Breitenecker \\
Editor-in-Chief & eic@ sne-journal.org
\end{tabular}

$\rightarrow$ www.sne-journal.org, $\quad$ 莑 office@sne-journal.org

\section{EUROSIM Congress and Conferences.}

Each year a major EUROSIM event takes place, the EUROSIM CONGRESS organised by a member society, SIMS EUROSIM Conference, and MATHM OD Vienna Conference (ASIM).

EUROSIM Congress 2019, the $10^{\text {th }}$ EUROSIM Congress, was organised by CEA-SM SG, the Spanish Simulation Society, in La Rioja, Logroño, Spain, July 1-5, 2019;

Due to Covid-19 virus in 2020 no EUROSIM events take place. To bridge this gap, EUROSIM is organising the series VESS - Virtual EUROSIM Simulation Seminar - seminars by simulation professionalists (2 hours via web), in preparation for upcoming EUROSIM events. $\rightarrow$ www.eurosim2023.eu

Next main event is SIMS EUROSIM Conference 2021, September 21-23, 2021, Oulu, Finland. SIMS, the Scandinavian simulation society, extends every third year the annual SIM S Conference to the SIMS EUROSIM Conference.

$\rightarrow$ www.scansims.org

MATHMOD Vienna. This triennial EUROSIM Conference is mainly organized by ASIM, the German simulation society, and ARGESIM, with main co-sponsor IFAC.

MATHM OD 2022, the $10^{\text {th }}$ MATHMOD Vienna Conference on Mathematical Modelling will take place in Vienna, Februray 16-18, 2022. $\rightarrow$ www.mathmod.at

EUROSIM Congress 2023, the $11^{\text {th }}$ EUROSIM Congress, will be organised by DBSS, the Dutch Benelux simulation society, in Amsterdam, Spring/Autumn 2023.

$\rightarrow$ www.eurosim2023.eu

Furthermore, EUROSIM Societies organize also local conferences, and EUROSIM co-operates with the organizers of the I3M Conference Series.

$\rightarrow$ www.liophant.org/conferences/ 


\section{EUROSIM Member Societies}

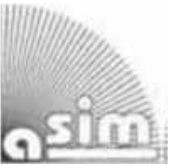

\section{ASIM \\ German Simulation Society \\ Arbeitsgemeinschaft Simulation}

ASIM (Arbeitsgemeinschaft Simulation) is the association for simulation in the German speaking area, servicing mainly Germany, Switzerland and Austria. ASIM was founded in 1981 and has now about 400 individual members (including associated), and 90 institutional or industrial members.

\section{$\rightarrow$ www.asim-gi.org with members' area \\ 拝=7info@asim-gi.org,admin@asim-gi.org \\ $\triangle$ ASIM - Inst. of Analysis and Scientific Computing Vienna University of Technology (TU Wien) \\ Wiedner Hauptstraße 8-10, 1040 Vienna, Austria}

\begin{tabular}{|c|c|}
\hline ASIM Officers & \\
\hline President & $\begin{array}{l}\text { Felix Breitenecker } \\
\text { felix.breitenecker@tuwien.ac.at }\end{array}$ \\
\hline \multirow[t]{3}{*}{ Vice presidents } & Sigrid Wenzel, s.wenzel@uni-kassel.de \\
\hline & T. Pawletta, thorsten.pawletta@hs-wismar.de \\
\hline & A. Körner, andreas.koerner@tuwien.ac.at \\
\hline Secretary & $\begin{array}{l}\text { Ch. Deatcu, christina.deatcu@ @s-wismar.de } \\
\text { I. Husinsky, Irmgard.husinsky@tuwien.ac.at }\end{array}$ \\
\hline \multirow{3}{*}{$\begin{array}{l}\text { Membership } \\
\text { Affairs }\end{array}$} & S.Wenzel, s.wenzel@uni-kassel.de \\
\hline & Ch. Deatcu, christina.deatcu@hs-wismar.de \\
\hline & F. Breitenecker, felix.breitenecker@tuwien.ac.at \\
\hline \multirow[t]{2}{*}{ Repr. EUROSIM } & F. Breitenecker, felix.breitenecker@tuwien.ac.at \\
\hline & A. Körner, andreas.koerner@tuwien.ac.at \\
\hline \multirow{2}{*}{$\begin{array}{l}\text { Intemat. Affairs } \\
\text { - GI Contact }\end{array}$} & O. Rose, Oliver.Rose@tu-dresden.de \\
\hline & N. Popper, niki.popper@dwh.at \\
\hline \multirow{2}{*}{$\begin{array}{l}\text { Editorial Board } \\
\text { SNE }\end{array}$} & T. Pawletta, thorsten.pawletta@hs-wismar.de \\
\hline & Ch. Deatcu, christina.deatcu@hs-wismar.de \\
\hline Web EuroSim & I. Husinsky, Irmgard.husinsky@tuwien.ac.at \\
\hline
\end{tabular}

ASIM is organising / co-organising the following international conferences:

- ASIM Int. Conference 'Simulation in Production and Logistics' - biannual

- ASIM 'Symposium Simulation Technique' - biannual

- MATHMOD Int. Vienna Conference on Mathmatical Modelling - triennial

Furthermore, ASIM is co-sponsor of WSC - Winter Simulation Conference, of SCS conferences SpringSim and SummerSim, and of I3M and Simutech conference series.

\begin{tabular}{|c|c|}
\hline \multicolumn{2}{|c|}{ ASIM Working Committees } \\
\hline GMMS & $\begin{array}{l}\text { Methods in Modelling and Simulation } \\
\text { Th. Pawletta, thorsten.pawletta@ hs-wismar.de }\end{array}$ \\
\hline SUG & $\begin{array}{l}\text { Simulation in Environmental Systems } \\
\text { Jochen Wittmann, } \\
\text { wittmann@informatik.uni-hamburg.de }\end{array}$ \\
\hline STS & $\begin{array}{l}\text { Simulation of Technical Systems } \\
\text { Walter Commerell, commerell@ hs-ulm.de }\end{array}$ \\
\hline SPL & $\begin{array}{l}\text { Simulation in Production and Logistics } \\
\text { Sigrid Wenzel, s.wenzel@ uni-kassel.de }\end{array}$ \\
\hline EDU & $\begin{array}{l}\text { Simulation in Education/Education in Simulation } \\
\text { A. Körner, andreas.koerner@tuwien.ac.at }\end{array}$ \\
\hline $\begin{array}{l}\text { BIG } \\
\text { DATA }\end{array}$ & $\begin{array}{l}\text { Working Group Data-driven Simulation in Life } \\
\text { Sciences; niki.popper@dwh.at }\end{array}$ \\
\hline $\begin{array}{l}\text { WORKING } \\
\text { GROUPS }\end{array}$ & $\begin{array}{l}\text { Simulation in Business Administration, in Traffic } \\
\text { Systems, for Standardisation, etc. }\end{array}$ \\
\hline
\end{tabular}

\section{CEA-SM SG - Spanish Modelling and Simulation Group}

CEA is the Spanish Society on Automation and Control and it is the national member of IFAC (International Federation of Automatic Control) in Spain. Since 1968 CEAIFAC looks after the development of the Automation in Spain, in its different issues: automatic control, robotics, SIMULATION, etc. The association is divided into national thematic groups, one of which is centered on Modeling, Simulation and Optimization, constituting the CEA Spanish Modeling and Simulation Group (CEA-SM SG). It looks after the development of the Modelling and Simulation (M\&S) in Spain, working basically on all the issues concerning the use of $M \& S$ techniques as essential engineering tools for decision-making and optimization.

$\rightarrow$ http://www.ceautomatica.es/grupos/

$\rightarrow$ emilio.jimenez@unirioja.es

simulacion@cea-ifac.es

$\triangle$ CEA-SMSG / Emilio Jiménez, Department of Electrical Engineering, University of La Rioja, San José de Calasanz 31, 26004 Logroño (La Rioja), SPAIN

\begin{tabular}{ll}
\hline CEA - SM SG Officers \\
\hline President & $\begin{array}{l}\text { Emilio Jiménez, } \\
\text { emilio.jimenez@ unirioja.es }\end{array}$ \\
\hline Vice president & $\begin{array}{l}\text { Juan Ignacio Latorre, } \\
\text { juanignacio.latorre@ unavarra.es }\end{array}$ \\
\hline Repr. EUROSIM & Emilio Jiménez, emilio.jimenez@unirioja.es \\
\hline Edit. Board SNE & Juan Ignacio Latorre, \\
& juanignacio.latorre@ unavarra.es \\
\hline Web EUROSIM & Mercedes Perez mercedes.perez@ unirioja.es \\
\hline
\end{tabular}




\section{Ca CSSS - Czech and Slovak csss Simulation Society}

CSSS -The Czech and Slovak Simulation Society has about 150 members working in Czech and Slovak national scientific and technical societies (Czech Society for Applied Cybernetics and Informatics, Slovak Society for Applied Cybernetics and Informatics). CSSS main objectives are: development of education and training in the field of modelling and simulation, organising professional workshops and conferences, disseminating information about modelling and simulation activities in Europe. Since 1992, CSSS is full member of EUROSIM.

$\rightarrow$ www.fit.vutbr.cz/CSSS

奉三” snorek@fel.cvut.cz

$\triangle$ CSSS / Miroslav Šnorek, CTU Prague

FEE, Dept. Computer Science and Engineering,

Karlovo nam. 13, 12135 Praha 2, Czech Republic

\begin{tabular}{ll}
\hline CSSS Officers & \\
\hline President & M iroslav Šnorek, snorek@fel.cvut.cz \\
\hline Vice president & M ikuláš Alexík, alexik@ frtk.fri.utc.sk \\
\hline Scientific Secr. & A. Kavička, Antonin.Kavicka@ upce.cz \\
\hline Repr. EuRoSım & M iroslav Šnorek, snorek@ fel.cvut.cz \\
\hline Edit. Board SNE & M ikuláŠ Alexík, alexik@ frtk.fri.utc.sk \\
\hline Web EuRoSım & Petr Peringer, peringer@ fit.vutbr.cz \\
\hline
\end{tabular}

\section{DBSS - Dutch Benelux Simulation Society}

The Dutch Benelux Simulation Society (DBSS) was founded in July 1986 in order to create an organisation of simulation professionals within the Dutch language area. DBSS has actively promoted creation of similar organisations in other language areas. DBSS is a member of EUROSIM and works in close cooperation with its members and with affiliated societies.

$\rightarrow$ www.DutchBSS.org

麦”a.w.heemink@its.tudelft.nl

$\triangle$ DBSS / A. W. Heemink

Delft University of Technology, ITS - twi,

Mekelweg 4, 2628 CD Delft, The Netherlands

DBSS Officers

\begin{tabular}{ll}
\hline President & M. Mujica M ota, m.mujica.mota@ hva.nl \\
\hline Vice president & A. Heemink, a.w.heemink@ its.tudelft.nl \\
\hline Treasurer & A. Heemink, a.w.heemink@its.tudelft.nl \\
\hline Secretary & P. M. Scala, p.m.scala@ hva.nl \\
\hline Repr. EuroSım & M. Mujica M ota, m.mujica.mota@ hva.nl \\
\hline Edit. SNE/Web & M. Mujica M ota, m.mujica.mota@ hva.nl
\end{tabular}

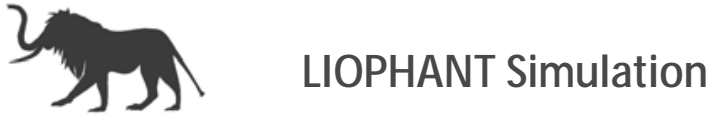

Liophant Simulation is a non-profit association born in order to be a trait-d'union among simulation developers and users; Liophant is devoted to promote and diffuse the simulation techniques and methodologies; the Association promotes exchange of students, sabbatical years, organization of International Conferences, courses and internships focused on $\mathrm{M} \& \mathrm{~S}$ applications.

$\rightarrow$ www.liophant.org

莑三”info@liophant.org

$\triangle$ LIOPHANT Simulation, c/o Agostino G. Bruzzone, DIME, University of Genoa, Savona Campus via Molinero 1, 17100 Savona (SV), Italy

LIOPHANT Officers

\begin{tabular}{ll}
\hline President & A.G. Bruzzone, agostino@itim.unige.it \\
\hline Director & E. Bocca, enrico.bocca@ liophant.org \\
\hline Secretary & A. Devoti, devoti.a@ iveco.com \\
\hline Treasurer & Marina M assei, massei@ itim.unige.it \\
\hline Repr. EuroSIM & A.G. Bruzzone, agostino@ itim.unige.it \\
\hline Deputy & F. Longo, f.longo@ unical.it \\
\hline Edit. Board SNE & F. Longo, f.longo@ unical.it \\
\hline Web EuRoSIM & F. Longo, f.longo@ unical.it \\
\hline
\end{tabular}

\section{LSS - Latvian Simulation Society}

The Latvian Simulation Society (LSS) has been founded in 1990 as the first professional simulation organisation in the field of Modelling and simulation in the post-Soviet area. Its members represent the main simulation centres in Latvia, including both academic and industrial sectors.

$\rightarrow$ www.itl.rtu.lv/imb/

麦引Egils.Ginters@rtu.lv

$\triangle$ Prof. Egils Ginters, Kirshu Str.13A, Cesis LV-4101, Latvia

\begin{tabular}{ll}
\hline LSS Officers & \\
\hline President & Yuri M erkuryev, merkur@itl.rtu.Iv \\
\hline Vice President & Egils Ginters, egils.ginters@ rtu.lv \\
\hline Secretary & Artis Teilans, artis.teilans@ rta.Iv \\
\hline Repr. EuROSIm & Egils Ginters, egils.ginters@ rtu.Iv \\
\hline Deputy & Artis Teilans, artis.teilans@ rta.Iv \\
\hline Edit. Board SNE & Juri Tolujew, Juri.Tolujew@iff.fraunhofer.de \\
\hline Web EuROSIm & Vitaly Bolshakov, vitalijs.bolsakovs@ rtu.Iv \\
\hline & \multicolumn{1}{r}{ Last data update November 2020 }
\end{tabular}




\section{KA-SIM Kosovo Simulation Society}

Kosova Association for Modeling and Simulation (KASIM, founded in 2009), is part of Kosova Association of Control, Automation and Systems Engineering (KACASE). KA-CASE was registered in 2006 as non Profit Organization and since 2009 is National Member of IFAC - International Federation of Automatic Control. KA-SIM joined EUROSIM as Observer Member in 2011. In 2016, KA-SIM became full member.

KA-SIM has about 50 members, and is organizing the international conference series International Conference in Business, Technology and Innovation, in November, in Durrhes, Albania, and IFAC Simulation Workshops in Pristina.

$\rightarrow$ www.ubt-uni.net/ka-case

拝”= ehajrizi@ubt-uni.net

$\triangle$ MOD\&SIM KA-CASE; Att. Dr. Edmond Hajrizi

Univ. for Business and Technology (UBT)

Lagjja Kalabria p.n., 10000 Prishtina, Kosovo

\begin{tabular}{ll}
\hline KA-SIM Officers & \\
\hline President & Edmond Hajrizi, ehajrizi@ ubt-uni.net \\
\hline Vice president & M uzafer Shala, info@ ka-sim.com \\
\hline Secretary & Lulzim Beqiri, info@ ka-sim.com \\
\hline Treasurer & Selman Berisha, info@ ka-sim.com \\
\hline Repr. EuRoSIm & Edmond Hajrizi, ehajrizi@ ubt-uni.net \\
\hline Deputy & M uzafer Shala, info@ ka-sim.com \\
\hline Edit. Board SNE & Edmond Hajrizi, ehajrizi@ ubt-uni.net \\
\hline Web EuRoSIM & Betim Gashi, info@ ka-sim.com \\
\hline
\end{tabular}

\section{NSSM - National Society for Simulation M odelling (Russia)}

NSSM - The Russian National Simulation Society (Национальное Общество Имитационного Моделирования - НОИМ) was officially registered in Russian Federation on February 11, 2011. In February 2012 NSS has been accepted as an observer member of EUROSIM, and in 2015 NSSM has become full member.

$\rightarrow$ www.simulation.su

莑”y yusupov@iias.spb.su

$\triangle$ NSSM / R. M. Yusupov,

St. Petersburg Institute of Informatics and Automation RAS, 199178, St. Petersburg, 14th lin. V.O, 39

\begin{tabular}{ll}
\hline NSSM Officers & \\
\hline President & R. M. Yusupov, yusupov@iias.spb.su \\
\hline Chair Man. Board & A. Plotnikov, plotnikov@sstc.spb.ru \\
\hline Secretary & M. Dolmatov, dolmatov@simulation.su \\
\hline Repr. EuRoSıM & $\begin{array}{l}\text { R.M. Yusupov, yusupov@iias.spb.su } \\
\text { Y. Senichenkov, } \\
\text { senyb@dcn.icc.spbstu.ru }\end{array}$ \\
\hline Deputy & B. Sokolov, sokol@iias.spb.su \\
\hline Edit. Board SNE & $\begin{array}{l}\text { Y. Senichenkov, senyb @ mail.ru, } \\
\text { senyb@dcn.icc.spbstu.ru, }\end{array}$ \\
\hline & \multicolumn{1}{c}{ Last data update February 2018 }
\end{tabular}

\section{PSCS - Polish Society for Computer Simulation}

PSCS was founded in 1993 in Warsaw. PSCS is a scientific, non-profit association of members from universities, research institutes and industry in Poland with common interests in variety of methods of computer simulations and its applications. At present PSCS counts 257 members.

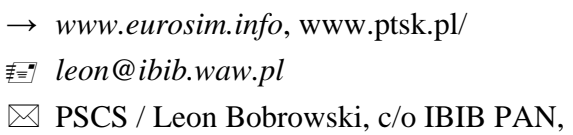

ul. Trojdena 4 (p.416), 02-109 Warszawa, Poland

\begin{tabular}{ll}
\hline PSCS Officers & \\
\hline President & Leon Bobrowski, leon@ibib.waw.pl \\
\hline Vice president & Tadeusz Nowicki, \\
& Tadeusz.Nowicki@ wat.edu.pl \\
\hline Treasurer & Z. Sosnowski, zenon@ii.pb.bialystok.pl \\
\hline Secretary & Zdzislaw Galkowski, \\
& Zdzislaw.Galkowski@simr.pw.edu.pl \\
\hline Repr. EuroSım & Leon Bobrowski, leon@ ibib.waw.pl \\
\hline Deputy & Tadeusz Nowicki, tadeusz.nowicki@wat.edu.pl \\
\hline Edit. Board SNE & Zenon Sosnowski, z.sosnowski@ pb.ed.pl \\
\hline Web EuroSIm & Magdalena Topczewska \\
& m.topczewska@pb.edu.pl \\
\hline
\end{tabular}




\section{SIMS - Scandinavian Simulation Society}

SIMS is the Scandinavian Simulation Society with members from the five Nordic countries Denmark, Finland, Iceland, Norway and Sweden. The SIMS history goes back to 1959. SIMS practical matters are taken care of by the SIMS board consisting of two representatives from each Nordic country (Iceland one board member).

SIMS Structure. SIMS is organised as federation of regional societies. There are FinSim (Finnish Simulation Forum), MoSis (Society for Modelling and Simulation in Sweden), DKSIM (Dansk Simuleringsforening) and NFA (Norsk Forening for Automatisering).

$\rightarrow \quad$ www.scansims.org

奉”bernt.lie@usn.no

$\triangle$ SIMS / Bernt Lie, Faculty of Technology, Univ.College of Southeast Norway, Department of Technology, Kjølnes ring 56, 3914 Porsgrunn, Norway

\begin{tabular}{ll}
\hline SIMS Officers & \\
\hline President & Bernt Lie, Bernt.Lie@ usn.no \\
\hline Vice president & Erik Dahlquist, erik.dahlquist@ mdh.se \\
\hline Treasurer & $\begin{array}{l}\text { Vadim Engelson, } \\
\text { vadime@ mathcore.com }\end{array}$ \\
\hline Repr. EuRoSIm & Esko Juuso, esko.juuso@ oulu.fi \\
\hline Edit. Board SNE & Esko Juuso, esko.juuso@ oulu.fi \\
\hline Web EuRoSIm & $\begin{array}{l}\text { Vadim Engelson, } \\
\text { vadime@ mathcore.com }\end{array}$ \\
\hline
\end{tabular}

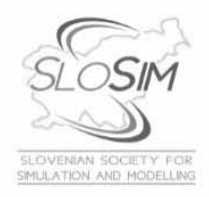

\section{SLOSIM - Slovenian Society for Simulation and Modelling}

SLOSIM - Slovenian Society for Simulation and Modelling was established in 1994 and became the full member of EUROSIM in 1996. Currently it has 90 members from both Slovenian universities, institutes, and industry. It promotes modelling and simulation approaches to problem solving in industrial as well as in academic environments by establishing communication and cooperation among corresponding teams.

$\rightarrow$ www.slosim.si

奉”slosim@fe.uni-lj.si

$\triangle$ SLOSIM / Vito Logar, Faculty of Electrical Engineering, University of Ljubljana, Tržaška 25, 1000 Ljubljana, Slovenia

\begin{tabular}{ll}
\hline \multicolumn{2}{l}{ SLOSIM Officers } \\
\hline President & Vito Logar, vito.logar@fe.uni-lj.si \\
\hline Vice president & Božidar Šarler, bozidar.sarler@ung.si \\
\hline Secretary & Simon Tomažič, simon.tomazic@fe.uni-lj.si \\
\hline Treasurer & Milan Simčič, milan.simcic@fe.uni-lj.si \\
\hline Repr. EuRoSıM & B. Zupančič, borut.zupancic@fe.uni-lj.si \\
\hline Deputy & Vito Logar, vito.logar@fe.uni-lj.si \\
\hline Edit. Board SNE & R. Karba, rihard.karba@fe.uni-lj.si \\
\hline Web EuRoSIM & Vito Logar, vito.logar@fe.uni-lj.si \\
\hline
\end{tabular}

\section{UKSIM - United Kingdom Simulation Society}

The UK Simulation Society is very active in organizing conferences, meetings and workshops. UKSim holds its annual conference in the March-April period. In recent years the conference has always been held at Emmanuel College, Cambridge. The Asia Modelling and Simulation Section (AMSS) of UKSim holds 4-5 conferences per year including the EMS (European Modelling Symposium), an event mainly aimed at young researchers, organized each year by UKSim in different European cities. Membership of the UK Simulation Society is free to participants of any of our conferences and their co-authors.

$\rightarrow$ uksim.info

恝”david.al-dabass@ntu.ac.uk

\begin{tabular}{|c|c|}
\hline $\begin{array}{l}\triangle \text { UKSIM / } \\
\text { Computing \& Infor } \\
\text { Nottingham Trent } \\
\text { Clifton lane, Nottin } \\
\text { domUKSIM Officer }\end{array}$ & $\begin{array}{l}\text { Prof. David Al-Dabass } \\
\text { matics, } \\
\text { University } \\
\text { gham, NG11 8NS, United King- } \\
\text { s }\end{array}$ \\
\hline President & $\begin{array}{l}\text { David Al-Dabass, } \\
\text { david.al-dabass@ntu.ac.uk }\end{array}$ \\
\hline Secretary & T. Bashford,tim.bashford@uwtsd.ac.uk \\
\hline Treasurer & D. Al-Dabass, david.al-dabass@ntu.ac.uk \\
\hline $\begin{array}{l}\text { Membership } \\
\text { chair }\end{array}$ & G. Jenkins, glenn.l.jenkins@smu.ac.uk \\
\hline Local/Venue chair & Richard Cant, richard.cant@ntu.ac.uk \\
\hline Repr. EURoSIM & Dr Taha Osman, taha.osman@ntu.ac.uk \\
\hline Deputy & T. Bashford,tim.bashford@uwtsd.ac.uk \\
\hline Edit. Board SNE & D. Al-Dabass, david.al-dabass@ntu.ac.uk \\
\hline
\end{tabular}




\section{EUROSIM Observer Members}

\section{ROMSIM - Romanian Modelling and Simulation Society}

ROMSIM has been founded in 1990 as a non-profit society, devoted to theoretical and applied aspects of modelling and simulation of systems. ROMSIM currently has about 100 members from Romania and Moldavia.

$\rightarrow$ www.eurosim.info/societies/romsim/

莑=7florin_h2004@yahoo.com

$\triangle$ ROMSIM / Florin Hartescu,

National Institute for Research in Informatics, Averescu

Av. 8 - 10, 011455 Bucharest, Romania

\begin{tabular}{ll}
\hline ROMSIM Officers \\
\hline President & N. N. \\
\hline Vice president & $\begin{array}{l}\text { Florin Hartescu, } \\
\text { florin_h2004@yahoo.com } \\
\text { Marius Radulescu, } \\
\text { mradulescu.csmro@yahoo.com }\end{array}$ \\
\hline Repr. EuRoSIM & $\begin{array}{c}\text { Marius Radulescu, } \\
\text { mradulescu.csmro@yahoo.com }\end{array}$ \\
\hline Deputy & $\begin{array}{l}\text { Florin Hartescu, } \\
\text { florin_h2004@yahoo.com }\end{array}$ \\
\hline Edit. Board SNE & Constanta Zoe Radulescu, zoe@ ici.ro \\
\hline Web EuRoSim & $\begin{array}{l}\text { Florin Hartescu, } \\
\text { florin_h2004@yahoo.com }\end{array}$ \\
\hline &
\end{tabular}

\section{ALBSIM - Albanian Simulation Society}

The Albanian Simulation Society has been initiated at the Department of Statistics and Applied Informatics, Faculty of Economy at the University of Tirana, by Prof. Dr. Kozeta Sevrani.

The society is involved in different international and local simulation projects, and is engaged in the organisation of the conference series ISTI - Information Systems and Technology. In July 2019 the society was accepted as EUROSIM Observer Member.

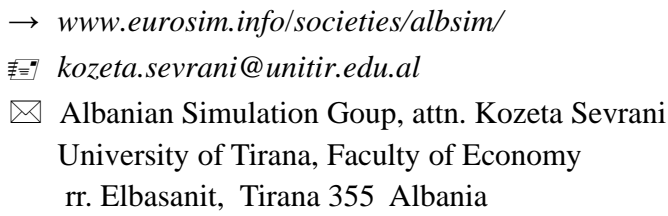

\begin{tabular}{lc}
\hline Albanian Simulation Society- Officers \\
\hline Chairt & $\begin{array}{c}\text { Kozeta Sevrani, } \\
\text { kozeta.sevrani@unitir.edu.al }\end{array}$ \\
\hline Repr. EuroSim & $\begin{array}{c}\text { Kozeta Sevrani, } \\
\text { kozeta.sevrani@unitir.edu.al }\end{array}$ \\
\hline Edit. Board SNE & $\begin{array}{c}\text { Albana Gorishti, } \\
\text { albana.gorishti@ unitir.edu.al } \\
\text { Majlinda Godolja, } \\
\text { majlinda.godolja@feut.edu.al }\end{array}$ \\
& Last data update July 2019
\end{tabular}

\section{Societies in Re-Organisation / Former Societies}

The following societies are at present inactive or under re-organisation:

- CROSSIM - Croatian Society for Simulation Modelling

Contact: Tarzan Legović,Tarzan.Legovic@irb.hr

- FranCOSim - Société Francophone de Simulation

- HSS - Hungarian Simulation Society

- ISCS - Italian Society for Computer Simulation

The following societies have been formally terminated:

- $\quad$ MIMOS -Italian Modeling \& Simulation Association; terminated end of 2020 . 


\section{Association Simulation News}

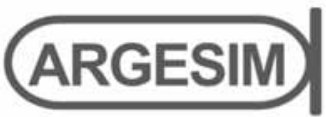

ARGESIM is a non-profit association generally aiming for dissemination of information on system simulation from research via development to applications of system simulation. ARGESIM is closely co-operating with EUROSIM, the Federation of European Simulation Societies, and with ASIM, the German Simulation Society. ARGESIM is an 'outsourced' activity from the Mathematical Modelling and Simulation Group of TU Wien, there is also close co-operation with TU Wien (organisationally and personally).

$\rightarrow$ www.argesim.org

奉: $\rightarrow$ office@argesim.org

$\bowtie \rightarrow$ ARGESIM/Math. Modelling \& Simulation Group, Inst. of Analysis and Scientific Computing, TU Wien Wiedner Hauptstrasse 8-10, 1040 Vienna, Austria Attn. Prof. Dr. Felix Breitenecker

ARGESIM is following its aims and scope by the following activities and projects:

- Publication of the scientific journal SNE -

Simulation Notes Europe (membership journal of EUROSIM, the Federation of European Simulation Societies) - www.sne-journal.org

- Organisation and Publication of the ARGESIM Benchmarks for Modelling Approaches and Simulation Implementations

- $\quad$ Publication of the series ARGESIM Reports for monographs in system simulation, and proceedings of simulation conferences and workshops

- Publication of the special series FBS Simulation Advances in Simulation / Fortschrittsberichte Simulation - monographs in co-operation with ASIM, the German Simulation Society

- Support of the Conference Series MATHMOD Vienna (triennial, in co-operation with EUROSIM, ASIM, and TU Wien) - www.mathmod.at

- Administration of ASIM (German Simulation Society) and administrative support for EUROSIM www.eurosim.info

- Simulation activities for TU Wien

ARGESIM is a registered non-profit association and a registered publisher: ARGESIM Publisher Vienna, root ISBN 978-3-901608-xx-y, root DOI 10.11128/z...zz.zz. Publication is open for ASIM and for EUROSIM Member Societies.

\section{SNE - Simulation Notes Europe}

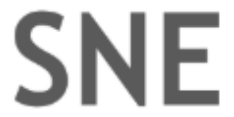

The scientific journal SNE - Simulation Notes Europe provides an international, high-quality forum for presentation of new ideas and approaches in simulation - from modelling to experiment analysis, from implementation to verification, from validation to identification, from numerics to visualisation - in context of the simulation process. SNE puts special emphasis on the overall view in simulation, and on comparative investigations.

Furthermore, SNE welcomes contributions on education in/for/with simulation.

SNE is also the forum for the ARGESIM Benchmarks on Modelling Approaches and Simulation Implemen-tations publishing benchmarks definitions, solutions, reports and studies - including model sources via web.

$\rightarrow$ www.sne-journal.org,

拝=゙ $\rightarrow$ office@sne-journal.org,eic@sne-journal.org

$\triangle \rightarrow$ SNE Editorial Office

ARGESIM/Math. Modelling \& Simulation Group, Inst. of Analysis and Scientific Computing, TU Wien Wiedner Hauptstrasse 8-10, 1040 Vienna, Austria EiC Prof. Dr. Felix Breitenecker

SNE, primarily an electronic journal, follows an open access strategy, with free download in basic layout. SNE is the official membership journal of EUROSIM, the Federation of European Simulation Societies. Members of EUROSIM Societies are entitled to download SNE in highquality, and to access additional sources of benchmark publications, model sources, etc. On the other hand, SNE offers EUROSIM Societies a publication forum for postconference publication of the society's international conferences, and the possibility to compile thematic or event-based SNE Special Issues.

Simulationists are invited to submit contributions of any type - Technical Note, Short Note, Project Note, Educational Note, Benchmark Note, etc. via SNE's website:

\section{CNE SIMULATION $\checkmark$ N NOTES EUROPE}

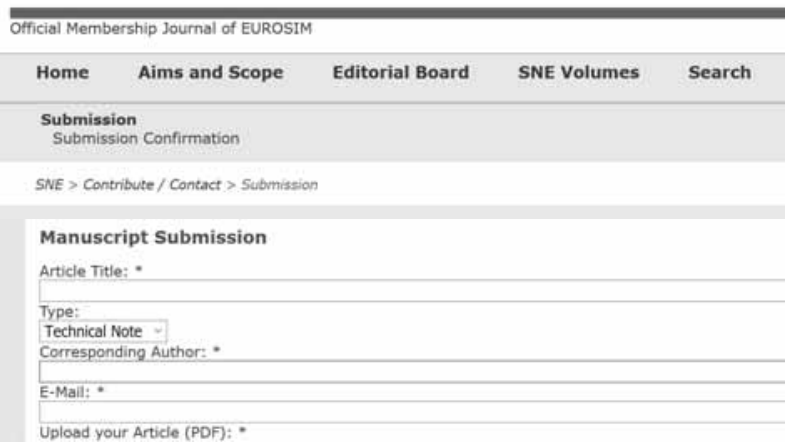




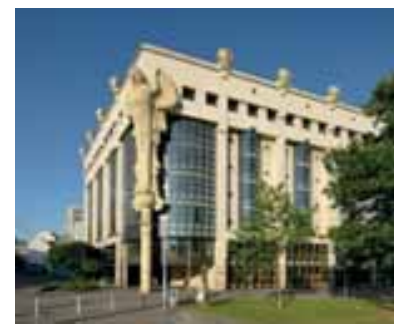

February 16-18, 2022

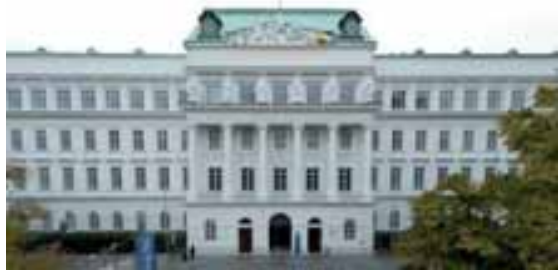

The scope of MATHMOD 2022 covers theoretic and applied aspects of various types of mathematical modelling (e.g., equations of various types, automata, Petri nets, bond graphs, qualitative and fuzzy models, machine learning) for systems of dynamic nature (deterministic, stochastic, continuous, discrete or hybrid with respect to time).

The topics to be discussed include e.g.

- modelling theory; first-principles, identification, optimization, order reduction and validation

- automation of modelling and software tools

- computer based modelling, modelling for/by simulation, co-simulation, modelling standards

- qualitative, modular, interdisciplinary modelling

- comparison of methods for modelling, alternative modelling methods (CAS, fuzzy, NN, QSS, etc.)

- machine learning and artificial intelligence for modelling

- model analysis and calibration, effects of modelling errors on overall performance of an engineering system

- applications in the field of engineering systems and in natural sciences

- applications in environmental systems, biotechnology, etc.

- applications in operation research, logistics and planning

- applications in medicine, physiology, health care and health systems

- education in/for/with modelling

- modelling aspects in scientific computing

- modelling for control and real-time applications

- machine learning, data analytics, big data

\section{TYPES OF CONTRIBUTIONS}

MATHMOD 2022 invites to submit contributions of following types:

- MATHMOD Full Contribution

6 pages, full paper peer review;

publication in MATHMOD 2022 Full Contribution Proceedings at IFAC-PapersOnLine

(MATHMOD Volume ISBN and individual paper DOI);

oral presentation in thematic sessions or minisymposia

- MATHMOD Discussion Contribution

2 pages extended abstract, abstract review;

publication in MATHMOD 2022 Discussion Contribution Proceedings in ARGESIM Report

(electronic; MATHMOD Volume ISBN and individual paper DOI)

short oral presentation with poster display or oral presentation in minisymposium

\section{MATHMOD MINISYM POSIA}

Established scientists and engineers in mathematical modelling are encouraged to organise MATHMOD Minisymposia by inviting contributions to a special topic of interest (Full Contributions or Discussion Contributions). Details for organisation of minisymposia can be found at the MATHMOD website www.mathmod.at.

\section{CONFERENCE PUBLICATIONS}

The MATHMOD Conference Proceedings (Full Contributions) are published in the IFAC-PapersOnLine series hosted at the ScienceDirect web service. A MATHMOD Abstract Preprint Volume with abstracts of all contribution types will be available electronically at the conference. Furthermore, following the IFAC copyright regulations, suitably adapted versions of MATHMOD Contributions which contain sufficiently new material may be submitted to the Journal of Mathematical and Computer Modelling of Dynamical Systems (MCMDS), published by Taylor and Francis. 


\section{Schedule for EUROSIM Conferences and Congress}

EUROSIM societies organise virtual conferences 2020 - 2021, and from 2022, the simulation community hopes to meet again at conferences as they used to be: Face-to-Face conferences.

\begin{tabular}{|c|c|c|}
\hline$* \mathrm{SS}_{*}^{*}$ & 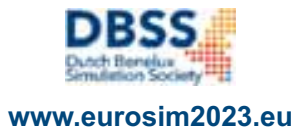 & $\begin{array}{l}\text { VESS - VIRTUAL EUROSIM SIMULATION SEMINAR } \\
\text { free online seminar series }\end{array}$ \\
\hline
\end{tabular}

The EUROSIM Board and DBSS started in June 2020 VESS - the Virtual EUROSIM Seminar, a series of online presentations discussing trends in modelling and simulation. These international online simulation seminars - monthly or bimonthly - are open to everybody, via Zoom, lasting 60 minutes (45 minutes presentations, 15 minutes Q \& A).

Information and informal registration via website www.eurosim2023.eu

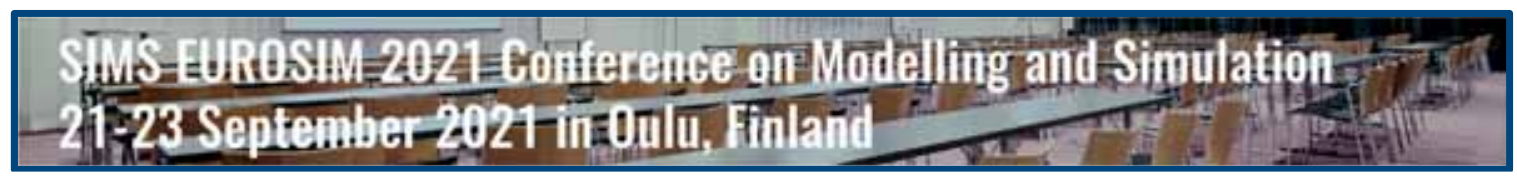

The First SIMS EUROSIM Conference on Modelling and Simulation, SIMS EUROSIM 2021 takes place in Oulu, Finland, September 21-23, 2021. The 62nd International Conference of Scandinavian Simulation Soci ety, SIMS 2021, is embedded with SIMS EUROSIM 2021. The SIMS EUROSIM conference will be organized every third year by SIMS and EUROSIM. The background of this conference series is in the 60-years history of Scandinavian Simulation Society, SIMS.

SIMS EUROSIM 2021 will be organised as virtual conference - info www.scansims.org

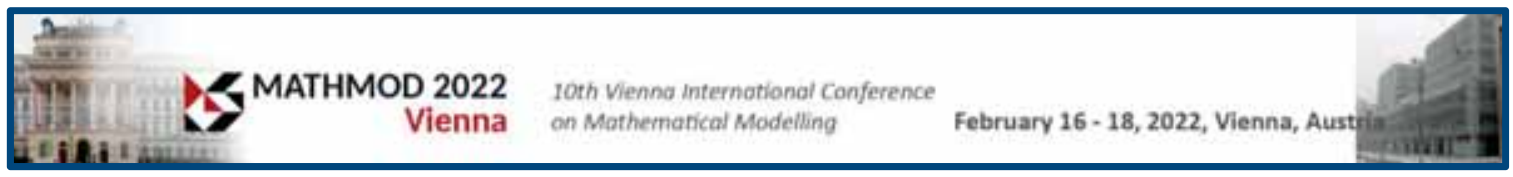

MATHMOD organizers continue the conference series one year later, with 10th MATHMOD 2022, February 16-18, 2022, as F2F event. MATHMOD 2022, one of EUROSIM's main events, provides a forum for professionals, researchers, and experts in the field of theoretic and applied aspects of mathematical modelling for systems of dynamic nature.

The scope of the MATHMOD 2022 conference covers theoretic and applied aspects of various types of mathematical modelling (equations of various types, automata, Petri nets, bond graphs, qualitative and fuzzy models) for systems of dynamic nature (deterministic, stochastic, continuous, discrete or hybrid) - info and details www.mathmod.at

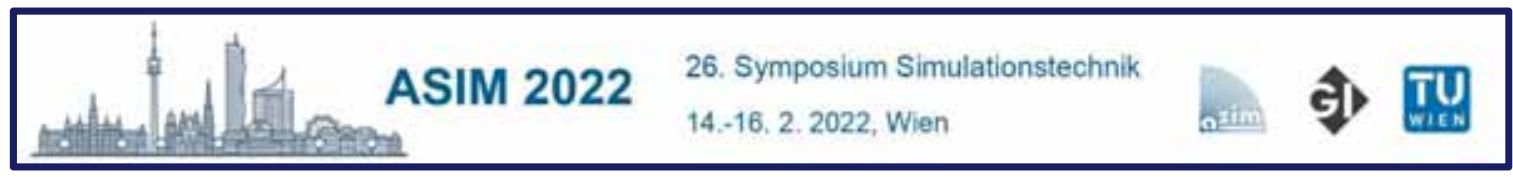

ASIM - the German / Austrian / Swiss simulation society - is organising the 26th Symposium Simulation Technique ASIM 2022 at TU Vienna, February 14-16, just before MATHMOD 2022. ASIM hopes for a German/English-based event as it used to be before - with personal contacts, and in synergy with MATHMOD 2022. - info www.asim-gi.org/asim2022

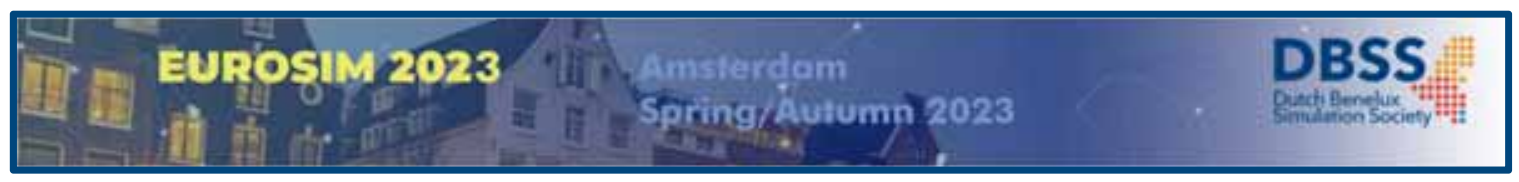

EUROSIM 2023, the 11th EUROSIM Congress, will take place in Amsterdam, The Netherlands, Spring/Autumn 2023. It will be organized by the Dutch Benelux Simulation Society (www.dutchbss.org) supported mainly by their corporate members like TU Delft, Amsterdam University of Applied Sciences, EUROCONTROL and IGAMT (www.igamt.eu).

Due to the growth of Simulation and its relationship with other analytical techniques like Big Data, Al, Machine Learning, Large Scale Simulation and others, the event will be structured, for the first time, in dedicated tracks focused on different areas and applications of Simulation ranging from aviation to health care and humanitarian activities.

Please follow the news and activities towards the EUROSIM 2023 at www.eurosim2023.eu 